This is the peer reviewed version of the following article: Angew. Chem., Int. Ed. 2010, 49, 5767, which has been published in final form at http://onlinelibrary.wiley.com/doi/10.1002/anie.201001853/abstract. This article may be used for non-commercial purposes in accordance With Wiley-VCH Terms and Conditions for self-archiving

\title{
Catalytic Selective Cyclizations of Aminocyclopropanes: Formal Synthesis of Aspidospermidine and Total Synthesis of Goniomitine.**
}

\author{
Filippo De Simone, Jürg Gertsch and Jérôme Waser*
}

((Dedication----optional))

Polyheterocyclic structures are present in most natural and synthetic molecules with important biological activity. ${ }^{[1]}$ Therefore, the discovery of new efficient cyclization reactions is important to access natural products and to explore a broad range of complex scaffolds with potentially enhanced bioactivity. ${ }^{[2]}$ We have recently reported the first catalytic formal homo-Nazarov cyclization of vinyl cyclopropyl ketones for the synthesis of cyclohexenones (A, Scheme 1) ${ }^{[3]}$ In contrast to the well-established Nazarov cyclization of divinyl ketones to give cyclopentenones ${ }^{[4]}$ examples of homoNazarov cyclizations are rare and require stoichiometric amounts of strong Lewis acids and/or high temperatures. ${ }^{[5]}$ The mild catalytic conditions developed in our work allowed us to apply our method to several unprecedented heterocyclic structures, but the scope of the reaction was limited by the required presence of an electron-rich aromatic group in order to stabilize the formed carbocationic intermediate $\mathbf{I}$.

A heteroatom should also be able to stabilize the formed carbocation, as demonstrated by the rich chemistry of donoracceptor cyclopropanes. ${ }^{[6]}$ Aminocyclopropanes in particular may lead to the fused amino-cyclohexane core of numerous biologically relevant alkaloids $\left(\mathrm{R}^{1}=\mathrm{N}\right.$ in Scheme 1$)$. Cyclization of an acyl indole substituted amino cyclopropane $\mathbf{1}$ would constitute a general entry into the Aspidosperma alkaloids, such as aspidospermidine (2) (B in Scheme 1). The tetracyclic core obtained in the cyclization is not only present in the Aspidosperma family, but also in more complex natural products such as vinblastine or vincristine, which

[*] F. De Simone and Prof. Dr. J. Waser Laboratory of Catalysis and Organic Synthesis

Ecole Polytechnique Fédérale de Lausanne

EPFL SB ISIC LCSO, BCH 4306, 1015 Lausanne $(\mathrm{CH})$

Fax: (+)4121 6939700

E-mail: jerome.waser@epfl.ch

Homepage: http://isic.epfl.ch/lcso

Prof. Dr. Jürg Gertsch

Institute of Biochemistry and Molecular Medicine

Bühlstrasse 28, CH-3012 Bern $(\mathrm{CH})$

[**] The EPFL is acknowledged for financial support. Miss Letitzia Gullifa (EPFL) is acknowledged for the preparation of starting material, Dr. Rosario Scopelliti (EPFL) for the Xrays studies, Dr. Markus Wartmann (Novartis AG) and Dr. Stefanie Krämer (ETH Zurich) for the gift of cancer cells and Prof. Brian L. Pagenkopf (University of Western Ontario) for a copy of the original NMR data for goniomitine (3). The collaboration between J. W. and J. G. was initiated with the support of COST CM0804 (Chemical Biology with Natural Products).

Supporting information for this article is available on the WWW under http://www.angewandte.org or from the author. are front-line drugs in cancer therapy. ${ }^{[7]}$ Although the combination of synthetically challenging structures and potential medical applications has resulted in a large number of successful total syntheses of aspidospermidine in the past, ${ }^{[8]}$ the development of more general and flexible synthetic approaches is still required in order to access new analogs. Herein, we report the first example of the formal homo-Nazarov cyclization of aminocyclopropanes and its application in the formal total synthesis of aspidospermidine (2). Additionally, we demonstrate how a simple modification in reaction conditions leads to the scaffold of goniomitine (3), an indole alkaloid isolated from the tree Gonioma malagasy, ${ }^{[9]}$ starting from aminocyclopropane 1. In contrast to the Aspidosperma scaffold, the goniomitine ring system is unique in natural products, and only two total syntheses have been reported so far. ${ }^{\left[{ }^{b}, 9 c\right]}$ Based on our cyclization strategy, an efficient total synthesis of goniomitine (3) was accomplished and we present here the first study of its bioactivity, revealing significant cytotoxicity against several cancer cell lines, including vinblastine and taxol resistant P-glycoprotein (Pgp, MDR-1) overexpressing cells.
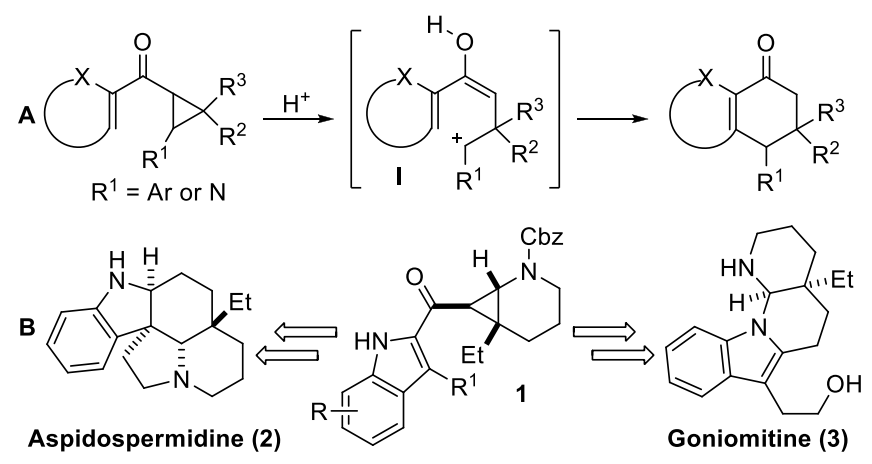

Scheme 1. Formal homo-Nazarov reaction and applications in the synthesis of polyheterocyclic natural products.

We began our research by examining the cyclization of a simple model system $\mathbf{4}$ containing the aminocyclopropane derived from the unsubstituted tetrahydro pyridine ring and a $\mathrm{N}$-methylindole (Scheme 2). ${ }^{[10]}$ Our standard conditions developed for the catalytic homo-Nazarov cyclization were highly successful and the reaction occurred in $90 \%$ yield with high diastereoselectivity for the cisfused product 5. ${ }^{[11]}$ This result demonstrated that carbamates were also excellent activating group for the cyclization reaction. 


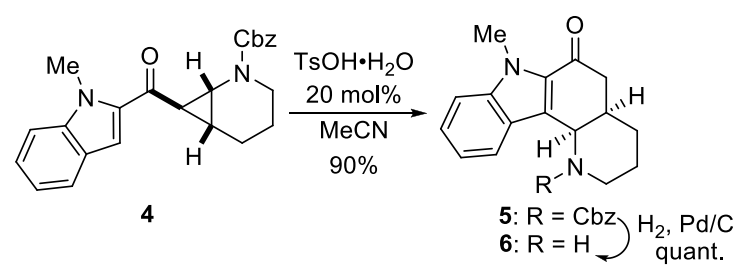

Scheme 2. Model study for the cyclization of aminocyclopropane 4.

Encouraged by this promising result, we then synthesized the ethyl substituted cyclopropane $\mathbf{1 2}$ required for the core of the Aspidosperma alkaloids (Scheme 3). The synthesis of carboxylic acid $\mathbf{1 0}$ was accomplished using a slightly modified procedure of Grieco. $^{[12]} \delta$-Valerolactam (7) was Cbz-protected and alkylated in one pot. Reduction and dehydratation led to dehydropiperidine 8 Enamide 8 was converted into aminocyclopropane 9 via cyclopropanation using CuOTf as catalyst. $^{[13]}$ The low diastereoselectivity observed in the cyclopropanation reaction is inconsequential, as the diastereomeric mixture of esters equilibrated to the exo isomer in presence of $\mathrm{BF}_{3} .{ }^{[12]}$ The exo ester obtained was hydrolyzed to obtain $\mathbf{1 0}$ as a pure diastereoisomer.

The choice of DMTMM ${ }^{[14]}$ to convert the sensitive cyclopropane $\mathbf{1 0}$ into Weinreb amide $\mathbf{1 1}$ in good yield was crucial. The coupling reaction between amide $\mathbf{1 1}$ and 2-lithiated $N$-methyl indole afforded the precursor $\mathbf{1 2}$ of the homo-Nazarov reaction. To our delight, our catalytic conditions for the cyclization gave the tetracyclic core of Aspidosperma alkaloids as a single diastereoisomer $\mathbf{1 3}$ after removal of the $\mathrm{Cbz}$ protecting group.

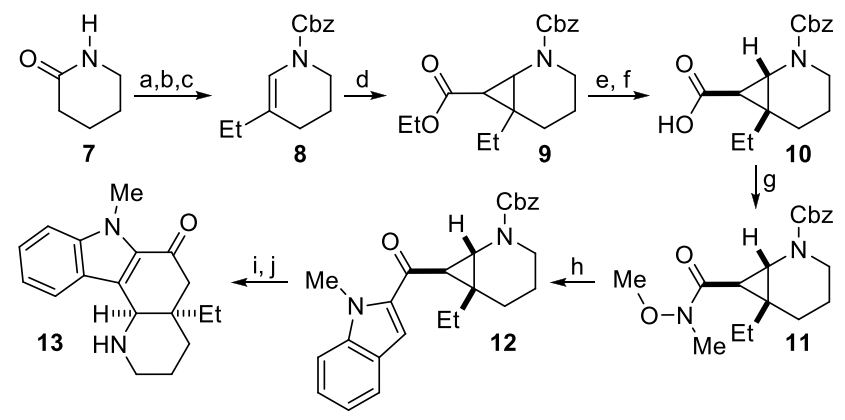

Scheme 3. Synthesis and cyclization of aminocyclopropane 12. Reagents and conditions: a) ${ }^{\mathrm{B}} \mathrm{BuLi}, \mathrm{CbzCl}$, Etl, THF, $-78^{\circ} \mathrm{C}, 67 \%$; b) $\mathrm{NaBH}_{4}, \mathrm{MeOH}$; c) $\mathrm{H}_{2} \mathrm{SO}_{4}$, THF, 93\% overall; d) (CuOTf) ${ }_{2} \cdot \mathrm{C}_{7} \mathrm{H}_{8}$, $\mathrm{N}_{2} \mathrm{CHCO}_{2} \mathrm{Et}, \mathrm{DCM}, 76 \%$ (dr: 1:1); e) $\mathrm{BF}_{3} \cdot \mathrm{Et}_{2} \mathrm{O}, \mathrm{DCM}$; f) $\mathrm{NaOH}$ $\mathrm{H}_{2} \mathrm{O} / \mathrm{THF} / \mathrm{EtOH}, 91 \%$ overall; e) DMTMM, NMM, THF, $\mathrm{MeNHOMe} \cdot \mathrm{HCl}, 93 \%$; h) $\mathrm{N}$-methylindole, ${ }^{n} \mathrm{BuLi}$, ${ }^{\mathrm{t}} \mathrm{BuOK}, \mathrm{THF}, 48 \%$; i) $\mathrm{TsOH}, \mathrm{MeCN}$, quant; j) $\mathrm{H}_{2}, \mathrm{Pd} / \mathrm{C}$, quant.

The high diastereoselectivity observed is an important advantage of the formal homo-Nazarov cyclization. Other approaches based on aminocyclopropanes in intermolecular Friedel-Crafts reactions used for the synthesis of Aspidosperma alkaloids proceeded with low selectivity. ${ }^{[8 \mathrm{~d}]}$ If an enantioselective cyclopropanation method can be developed, an asymmetric synthesis will become possible. ${ }^{[15]}$

For the synthesis of aspidospermidine (2) a free $\mathrm{NH}$ indole was required. Therefore, we investigated the use of bis-lithiated $\mathrm{N}$ carboxy indoles for the addition reaction to Weinreb amide $\mathbf{1 1}$ (Scheme 4). ${ }^{[16]}$ The interest of carbon dioxide as protecting/directing group resides in its easy removal from the product, which already occurs upon aqueous work-up. However, its use has been limited to simple substrates. ${ }^{[16]}$ We were therefore pleased to observe that the coupling procedure proceeded in moderate to good yields, leading directly to free NH indole 1a-c. This approach was highly convergent and allowed the easy variation of coupling partners for the synthesis of analogs.

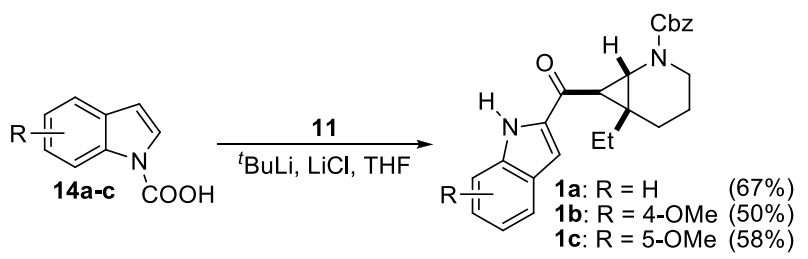

Scheme 4. Coupling of $\mathrm{N}$-carboxylated indoles.

The obtained product 1a was submitted to the standard conditions for the homo-Nazarov cyclization. We were surprised to observe the formation of two different products. The two compounds were identified as the desired product $\mathbf{1 5 a}$ resulting from cyclization on the $\mathrm{C} 3$ position of indole, and the compound 16a obtained via attack on the N1 position. The cyclic products were isolated in $74 \%$ yield and a ratio 15a to 16a of 1.6:1 (Table 1, Entry 1). In order to increase the selectivity for $\mathrm{C}-\mathrm{C}$ cyclization, we examined several Brønsted and Lewis acids as catalysts (Entries 14). The use of soft Lewis acids instead of Brønsted acids allowed the preferred formation of 15a as a pure diastereoisomer (Entries 2-3). Softer, milder Lewis acids could potentially exert an influence on the formation of the acyl iminium intermediate and favour the reaction of the softer C3 position of the indole ring. Deprotection of 15a gave the free amine, concluding the successful formal total synthesis of aspidospermidine (2), as this intermediate had already been reported by Wenkert. ${ }^{[8 \mathrm{~d}, 17]}$

The N-cyclization product 16a was also highly interesting, as it corresponded to the tetracyclic skeleton of goniomitine (3). When considering the rarity of this scaffold, the limited amount of synthetic approaches ${ }^{[9 b, 9 c]}$ and the absence of any study on his bioactivity, we found worthwhile to optimize the formation of the N1-cyclization product 16a. We hypothesized that the use of a less polar solvent for the cyclization reaction could enhance the reactivity of the iminium intermediate and favorize a fast attack on the harder N1 position. Indeed, a strong influence of solvents on the cyclization was observed in the presence of Brønsted acids (Table 1, Entries 5-8). We were delighted to isolate the goniomitine scaffold 16a in high yield and excellent selectivity using dichloromethane and toluene sulfonic acid as catalyst (Entry 9).

Cyclizations involving (acyl)iminium ions are important tools in the synthesis of alkaloids. ${ }^{[18]}$ Examples involving a possible competition between $\mathrm{N} 1$ and $\mathrm{C} 3$ cyclization are rare, and it is difficult to control the regioselectivity and stereoselectivity of these reactions. ${ }^{[19]}$ The ring-opening of aminocyclopropanes constitutes a new method for the generation of acyl iminium ions and the high level of control on the regio- and stereo- selectivity observed is unprecedented. To gain a first impression for the generality of the method, two methoxy indoles analogs $\mathbf{1 b}$ and $\mathbf{1 c}$ were examined, as similar electron-rich indoles are frequently encountered in natural products. Again, high yields and control of regioselectivity were achieved in these cases (Table 1, Entries 10-13).

Table 1. Cyclization of Aminocyclopropanes 1 


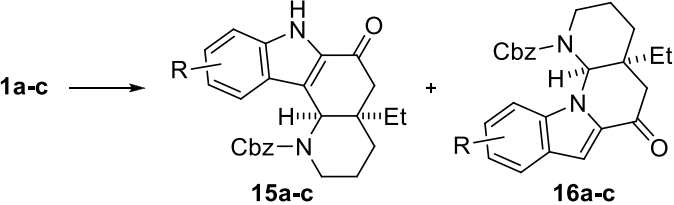

\begin{tabular}{llllll}
\hline Entry & $\mathrm{R}$ & Catalyst & Solvent & $\mathbf{1 5}: 16$ & Yield \\
\hline 1 & $\mathrm{H}$ & $\mathrm{TsOH}$ & $\mathrm{MeCN}$ & $1.6: 1^{[\mathrm{a}]}$ & 74 \\
2 & $\mathrm{H}$ & $\mathrm{Cu}(\mathrm{OTf})_{2}$ & $\mathrm{MeCN}$ & $8: 1^{[\mathrm{b}]}$ & n.d. \\
3 & $\mathrm{H}$ & $\mathrm{Pd}\left(\mathrm{CH}_{3} \mathrm{CN}\right)_{4}\left(\mathrm{BF}_{4}\right)_{2}$ & $\mathrm{MeCN}$ & $11: 1^{[\mathrm{b}]}$ & n.d. \\
4 & $\mathrm{H}$ & $\mathrm{Cu}(\mathrm{OTf})_{2}$ & $\mathrm{MeCN}$ & $7: 1^{[\mathrm{a}]}$ & 91 \\
5 & $\mathrm{H}$ & $\mathrm{TsOH}$ & $\mathrm{MeNO}_{2}$ & $1.3: 1^{[\mathrm{b}]}$ & n.d. \\
6 & $\mathrm{H}$ & $\mathrm{TsOH}$ & $\mathrm{THF}$ & polymers & n.d. \\
7 & $\mathrm{H}$ & $\mathrm{TsOH}$ & $\mathrm{DCM}$ & $1: 18^{[\mathrm{b}]}$ & n.d. \\
8 & $\mathrm{H}$ & $\mathrm{TsOH}$ & $\mathrm{Toluene}$ & $1: 16^{[\mathrm{b}]}$ & n.d. \\
9 & $\mathrm{H}$ & $\mathrm{TsOH}$ & $\mathrm{DCM}$ & $1: 21^{[\mathrm{a}]}$ & 89 \\
10 & $4-\mathrm{OMe}$ & $\mathrm{TsOH}$ & $\mathrm{DCM}$ & $1: 22^{[\mathrm{a}]}$ & 92 \\
11 & $4-\mathrm{OMe}$ & $\mathrm{Cu}(\mathrm{OTf})_{2}$ & $\mathrm{MeCN}$ & $8: 1^{[\mathrm{a}]}$ & 88 \\
12 & $5-\mathrm{OMe}$ & $\mathrm{TsOH}$ & $\mathrm{DCM}$ & $1: 20^{[\mathrm{a}]}$ & 86 \\
13 & $5-\mathrm{OMe}$ & $\mathrm{Cu}(\mathrm{OTf})_{2}$ & MeCN & $8: 1^{[\mathrm{a}]}$ & 95
\end{tabular}

[a] Yields and ratios obtained from isolated products. Reaction run with 1a-c (40-100 mg, 90-250 $\mu \mathrm{mol}), 15-25 \mathrm{~mol} \%$ catalyst and at a concentration of $0.025 \mathrm{M}$. [b] Ratios calculated by integration of ${ }^{1} \mathrm{H}$ NMR peaks in the crude mixture. Reaction carried out on a $10 \mathrm{mg}(25$ $\mu \mathrm{mol}$ ) scale at a concentration of 0.025 M. [c] n.d. $=$ not determined.

To finish the total synthesis of goniomitine (3) starting from 16a, it would be necessary to introduce the lateral chain at $\mathrm{C} 3$ in presence of the sensitive aminal functionality. In order to avoid this difficult task, we envisaged a more convergent approach starting from cyclization precursor 1d (Scheme 5). Indole 1d was obtained from tryptophol via TIPS protection, carboxylation, lithiation and addition to Weinreb amide 11. ${ }^{[20]}$ Cyclopropane 1d was cyclized in presence of catalytic amount of $\mathrm{TsOH}$ affording the tetracyclic core $\mathbf{1 7}$ of goniomitine (3) in $93 \%$ yield. The carbonyl group was reduced to the alcohol and acetylated. The acetate and the benzyl carbamate were cleaved in one step through hydrogenolysis. Deprotection of the primary alcohol gave goniomitine (3) in $77 \%$ overall yield from 17. ${ }^{[21]}$ The total synthesis of $( \pm)$-goniomitine $(3)$ was accomplished in a longer linear sequence of 13 steps ( 5 purifications by column chromatography) with an overall yield of $11 \%$.

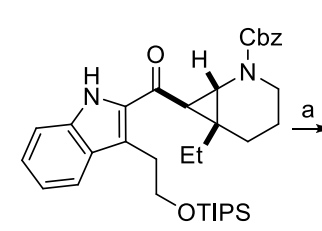

$1 d$

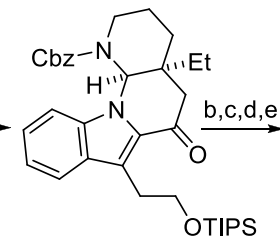

17

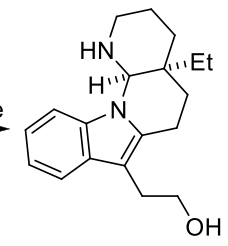

(士)-Goniomitine (3)
Scheme 5. Total synthesis of goniomitine (3). Reagents and conditions: a) $\mathrm{TsOH}, \mathrm{CH}_{2} \mathrm{Cl}_{2}, 93 \%$; b) $\mathrm{NaBH}_{4}, \mathrm{MeOH}$; c) $\mathrm{Ac}_{2} \mathrm{O}$, pyridine; d) $\mathrm{Pd} / \mathrm{C}, \mathrm{H}_{2}$, EtOH; e) TBAF, THF, $77 \%$ overall.

Somewhat surprisingly, we were unable to find any studies about the bioactivity of goniomitine (3). In a first biological assessment we therefore investigated the cytotoxicity of this natural product. Preliminary results are highly promising as goniomitine displays $\mathrm{nM}$ antiproliferative effects in several tumor cell lines
(Table 2). Interestingly, unlike taxol and vinblastine, which are approximately 100 -fold less effective in P-gp overexpressing cells (not shown), goniomitine did not lose its effect in the resistant MDR-1-MDCK cell line.

Table 2. Antiproliferative activity of goniomitine

\begin{tabular}{lc}
\hline \multicolumn{1}{c}{ Cell Lines } & IC $_{50}^{\left[{ }^{[a]}\right.}$ \\
\hline A549 & $205 \pm 27 \mathrm{nM}$ \\
MCF-7 & $239 \pm 13 \mathrm{nM}$ \\
HCT116 & $281 \pm 29 \mathrm{nM}$ \\
PC3 & $159 \pm 24 \mathrm{nM}$ \\
MDCK & $247 \pm 10 \mathrm{nM}$ \\
MDR-1-MDCK & $381 \pm 17 \mathrm{nM}$ \\
\hline
\end{tabular}

[a] $\mathrm{IC}_{50}$ values for inhibition of human tumor cell growth. A549: lung; MCF-7: breast; HCT-116: colon; PC-3M: prostate; MDCK canine kidney. MDR-1-MDCK is a human P-glycoprotein 170 (P-gp170)overexpressing multidrug-resistant cell line. ${ }^{[22]}$

In conclusion, we have demonstrated the versatility of aminocyclopropanes as (acyl)iminium precursors for intramolecular cyclizations. The reaction proceeded under mild conditions and control about the regioselectivity was possible by the right choice of catalyst and solvent. The power of the methodology has been demonstrated in the efficient formal total synthesis of aspidospermidine (2) and the total synthesis of goniomitine (3). Preliminary studies on the bioactivity of goniomitine revealed its relatively potent cytotoxicity (antiproliferative effect) (IC $\mathrm{I}_{50}$ : 150-400 $\mathrm{nM}$ ) against several tumor cell lines. Preliminary data show that this natural product disrupts the microtubule network (not shown). Therefore, goniomitine is a potential new anticancer lead structure. In the future, the high convergence of our synthetic approach will allow us to access a large number of analogs of goniomitine (3) for structure-activity relationship studies. Applications of aminocyclopropanes as iminium precursors for other types of cyclization or addition reactions as well as the development of asymmetric cyclopropanation methods for enamides are currently under investigation in our laboratory and the results of this work will be reported in due course.

Received: ((will be filled in by the editorial staff))

Published online on ((will be filled in by the editorial staff))

Keywords: Alkaloids · Aminocyclopropanes · Anticancer agents. Catalytic Cyclization · Heterocyclic compd · Regioselectivity.

[1] a) A. F. Pozharskii, A. R. Katritsky, A. T. Soldatenkov, in Heterocycles in Life and Society: An Introduction to Heterocyclic Chemistry and Biochemistry and the Role of Heterocycles in Science, Technology, Medicine and Agriculture, WILEY-VCH, Weinheim, 1997; b) J. Clardy, C. Walsh, Nature 2004, 432, 829.

[2] S. M. Ma, in Handbook of Cyclization Reactions, Wiley-VCH, 2009.

[3] F. De Simone, J. Andres, R. Torosantucci, J. Waser, Org. Lett. 2009, $11,1023$.

[4] a) I. N. Nazarov, I. I. Zaretskaya, Izv. Akad. Nauk. SSSR. Ser. Khim. 1941, 211; For reviews, see: b) K. L. Habermas, S. E. Denmark, T. K. Jones, Org. React. (N. Y.) 1994, 45, 1-158; c) A. J. Frontier, C. Collison, Tetrahedron 2005, 61, 7577; d) H. Pellissier, Tetrahedron 2005, 61, 6479; e) M. A. Tius, Eur. J. Org. Chem. 2005, 2193; f) W. Nakanishi, F. G. West, Curr. Opin. Drug Discovery Dev. 2009, 12, 732. 
[5] a) W. S. Murphy, S. Wattanasin, Tetrahedron Lett. 1980, 21, 1887; b) W. S. Murphy, S. Wattanasin, J. Chem. Soc. Perkin Trans. 1 1981, 2920; c) W. S. Murphy, S. Wattanasin, J. Chem. Soc. Perkin Trans. 1 1982, 1029; d) O. Tsuge, S. Kanemasa, T. Otsuka, T. Suzuki, Bull. Chem. Soc. Jpn. 1988, 61, 2897; e) V. K. Yadav, N. V. Kumar, Chem. Commun. 2008, 3774 .

[6] a) H. U. Reissig, R. Zimmer, Chem. Rev. 2003, 103, 1151; b) M. Yu B. L. Pagenkopf, Tetrahedron 2005, 61, 321; c) F. De Simone, J. Waser, Synthesis 2009, 3353.

[7] a) S. E. Malawista, H. Sato, K. G. Bensch, Science 1968, 160, 770; b B. Gigant, C. G. Wang, R. B. G. Ravelli, F. Roussi, M. O. Steinmetz, P. A. Curmi, A. Sobel, M. Knossow, Nature 2005, 435, 519; c) F. Gueritte, J. Fahy, The vinca Alkaloids, Anticancer agents from natural products, (Eds: D. J. C. Newman, G. M. Kingston) Taylor \& Francis group, 2005.

[8] For a few selected examples, see: a) G. Stork, J. E. Dolfini, J. Am Chem. Soc. 1963, 85, 2872; b) J. P. Kutney, Abdurahm.N, P. Lequesne, E. Piers, I. Vlattas, J. Am. Chem. Soc. 1966, 88, 3656; c) T. Gallagher, P. Magnus, J. C. Huffman, J. Am. Chem. Soc. 1982, 104 1140; d) E. Wenkert, T. Hudlicky, J. Org. Chem. 1988, 53, 1953; e) R. Iyengar, K. Schildknegt, J. Aube, Org. Lett. 2000, 2, 1625; f) M. A. Toczko, C. H. Heathcock, J. Org. Chem. 2000, 65, 2642; g) S. A. Kozmin, T. Iwama, Y. Huang, V. H. Rawal, J. Am. Chem. Soc. 2002, 124, 4628; h) L. A. Sharp, S. Z. Zard, Org. Lett. 2006, 8, 831; i) C. Sabot, K. C. Guerard, S. Canesi, Chem. Commun. 2009, 2941.

[9] Isolation: a) L. Randriambola, J. C. Quirion, C. Kanfan, H. P. Husson, Tetrahedron Lett. 1987, 28, 2123. Total syntheses: b) S. Takano, T. Sato, K. Inomata, K. Ogasawara, J. Chem. Soc. Chem. Commun. 1991 , 462; c) C. L. Morales, B. L. Pagenkopf, Org. Lett. 2008, 10, 157. Analogs studies: d) C. Hashimoto, H. P. Husson, Tetrahedron Lett. 1988, 29, 4563; e) G. Lewin, C. Schaeffer, Nat. Prod. Lett. 1995, 7 , 227; f) G. Lewin, C. Schaeffer, P. H. Lambert, J. Org. Chem. 1995, 60, 3282; g) G. Lewin, C. Schaeffer, R. Hocquemiller, E. Jacoby, S. Leonce, A. Pierre, G. Atassi, Heterocycles 2000, 53, 2353.
[10] See supporting information for the synthesis of 4.

[11] The cis stereochemistry was determined via 2D NMR experiments. See supporting information for details

[12] P. A. Grieco, M. D. Kaufman, J. Org. Chem. 1999, 64, 7586.

[13] R. Beumer, C. Bubert, C. Cabrele, O. Vielhauer, M. Pietzsch, O. Reiser, J. Org. Chem. 2000, 65, 8960.

[14] 4-(4,6-Dimethoxy-1,3,5-triazin-2-yl)- $N$-methylmorpholinium chloride.

[15] For a review on metal-catalyzed enantioselective cyclopropanation reactions, see: a) H. Pellissier, Tetrahedron 2008, 64, 7041. Highly selective methods for enamide substrates have not yet been reported. Alternatively, the kinetic resolution of aminocyclopropane esters can also be envisaged. ${ }^{[13]}$

[16] A. R. Katritzky, K. Akutagawa, Tetrahedron Lett. 1985, 26, 5935.

[17] The structure of deprotected $\mathbf{1 5 a}$ was definitively confirmed by X-rays diffractions study on the corresponding hydrochloride salt.

[18] B. E. Maryanoff, H. C. Zhang, J. H. Cohen, I. J. Turchi, C. A. Maryanoff, Chem. Rev. 2004, 104, 1431.

[19] a) A. Jackson, N. D. V. Wilson, A. J. Gaskell, J. A. Joule, J. Chem. Soc. C 1969, 2738; b) P. Forns, A. Diez, M. Rubiralta, J. Org. Chem. 1996, 61, 7882; c) M. Amat, M. Perez, N. Llor, C. Escolano, F. J. Luque, E. Molins, J. Bosch, J. Org. Chem. 2004, 69, 8681; d) S. Cutri, A. Diez, M. Bonin, L. Micouin, H. P. Husson, Org. Lett. 2005, 7, 1911.

[20] See supporting information

[21] The values of $400-\mathrm{MHz}{ }^{1} \mathrm{H}$ NMR spectra of goniomitine (3) were identical to those of natural ${ }^{[9 a]}$ and synthetic ${ }^{[9 b, 9 c]}$ goniomitine. The obtained values for ${ }^{13} \mathrm{C}$ NMR fitted perfectly with the reported values for synthetic goniomitine, but small differences were apparent when compared with natural goniomitine. A comparison of the spectra is provided in the supporting information.

[22] See supporting information for further details. See also: J. Gertsch, F. Feyen, A. Bützberger, B. Gerber, B. Pfeiffer, K. H. Altmann, Chembiochem 2009, 10, 2513. 


\section{Indole Alkaloids}

Filippo De Simone, Jürg Gertsch and Jérôme Waser Page -

Page

Catalytic Selective Cyclizations of Aminocyclopropanes: Formal Synthesis of Aspidospermidine and Total Synthesis of Goniomitine.
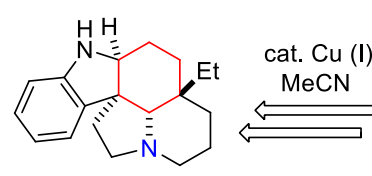

Aspidospermidine
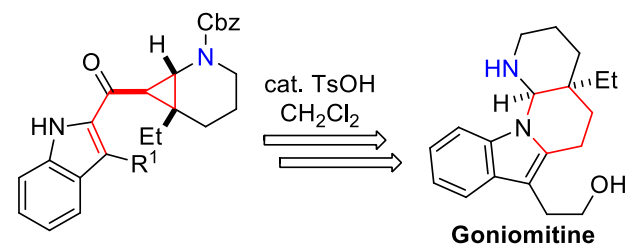

Mild Control: Selective cyclization of aminocyclopropanes on the N1 or C3 positions of indole was achieved by tuning the catalyst and solvent. The new strategy was applied to the formal synthesis of aspidospermidine and the total synthesis of goniomitine in 13 steps and $11 \%$ overall yield. The first studies on the bioactivity of goniomitine demonstrated significant cytotoxicity against several tumor cell lines (IC50: 150-400 nM). 


\section{Supporting Information}

1. General Methods

2. General procedures

3. Substrates synthesis

4. Total synthesis of goniomitine

5. Cell assays

6. Spectra
S2

$\mathrm{S} 2$

S4

S22

S26

S27 


\section{General Methods}

All reactions were carried out in oven dried glassware under an atmosphere of nitrogen, unless stated otherwise. For quantitative flash chromatography technical grade solvents were used. For flash chromatography for analysis, HPLC grade solvents from Sigma-Aldrich were used. THF, $\mathrm{Et}_{2} \mathrm{O}, \mathrm{CH}_{3} \mathrm{CN}_{\text {, }}$ toluene, hexane and $\mathrm{CH}_{2} \mathrm{Cl}_{2}$ were dried by passage over activated alumina under nitrogen atmosphere $\left(\mathrm{H}_{2} \mathrm{O}\right.$ content $<10$ ppm, Karl-Fischer titration). $\mathrm{NEt}_{3}$ and pyridine were distilled under nitrogen from $\mathrm{KOH}$. All chemicals were purchased from Acros, AldricH; Fluka, VWR, Aplichem or Merck and used as such unless stated otherwise. Chromatographic purification was performed as flash chromatography using MachereyNagel silica 40-63, $60 \AA$, using the solvents indicated as eluent with 0.1-0.5 bar pressure. The deactivation of silica was obtained with a $1 \%$ solution of $\mathrm{Et}_{3} \mathrm{~N}$ in the indicated solvent. TLC was performed on Merck silica gel $60 \mathrm{~F}_{254}$ TLC glass plates or aluminium plates and visualized with UV light, permanganate stain, CAN stain or Anisaldehyde stain. Melting points were measured on a Büchi B-540 melting point apparatus using open glass capillaries, the data is uncorrected. ${ }^{1} \mathrm{H}-\mathrm{NMR}$ spectra were recorded on a Brucker DPX-400 $400 \mathrm{MHz}$ and a Brucker AV-500 $500 \mathrm{MHz}$ spectrometer in chloroform-d, DMSO- $\mathrm{d}_{6}$ or $\mathrm{CD}_{3} \mathrm{OD}$, all signals are reported in ppm with the internal chloroform signal at $7.26 \mathrm{ppm}$, the internal DMSO signal at $2.50 \mathrm{ppm}$ or the internal methanol signal at $3.30 \mathrm{ppm}$ as standard. The data is being reported as $(\mathrm{s}=\operatorname{singlet}, \mathrm{d}=$ doublet, $\mathrm{t}=$ triplet, $\mathrm{q}=$ quadruplet, $\mathrm{q} i=$ quintet, $\mathrm{m}=$ multiplet or unresolved, $\mathrm{br}=$ broad signal, app $=$ apparent, coupling constant(s) in $\mathrm{Hz}$, integration; interpretation). ${ }^{13} \mathrm{C}-\mathrm{NMR}$ spectra were recorded with ${ }^{1} \mathrm{H}$ decoupling on a Brucker DPX-400 100 MHz and a Brucker AV-500 125 MHz spectrometer in chloroform$\mathrm{d}$, DMSO- $\mathrm{d}_{6}$ or $\mathrm{CD}_{3} \mathrm{OD}$, all signals are reported in ppm with the internal chloroform signal at $77.0 \mathrm{ppm}$, the internal DMSO signal at $39.5 \mathrm{ppm}$ or the internal methanol signal at $49.0 \mathrm{ppm}$ as standard. Infrared spectra were recorded on a JASCO FT-IR B4100 spectrophotometer with an ATR PRO410-S and a ZnSe prisma and are reported $\mathrm{as} \mathrm{cm}^{-1}(\mathrm{w}=$ weak, $\mathrm{m}=$ medium, $\mathrm{s}=$ strong, $\mathrm{br}=$ broad $)$. Gas chromatographic and low resolution mass spectrometric measurements were performed on a Perkin-Elmer Clarus 600 gas chromatographer and mass spectrometer using a Perkin-Elmer Elite fused silica column (length: $30 \mathrm{~m}$, diameter: $0.32 \mathrm{~mm}$ ) and Helium as carrier gas. High resolution mass spectrometric measurements were performed by the mass spectrometry service of ISIC at the EPFL on a MICROMASS (ESI) Q-TOF Ultima API. HPLC measurement were done on a JASCO HPLC system with an AS2055 Autosampler, a PU 2089 Pump, a UV 2075 detector and a SEDEX 85 (SEDERE) detector using a CHIRALPAK IC column from DAICEL Chemical Industries Ltd. HPLC grade solvents from Sigma-Aldrich were used.

\section{General Procedures}

\section{General procedure 1 (GP1): homo-Nazarov cyclization}


Toluenesulfonic acid (0.2 equiv) was added to a solution of vinyl cyclopropyl ketone (0.04 $\mathrm{M}$ in anhydrous $\mathrm{CH}_{3} \mathrm{CN}$ ) at room temperature. The reaction was stirred during the indicated time. The solution was quenched with $\mathrm{NaHCO}_{3}$ and extracted with $\mathrm{Et}_{2} \mathrm{O}$. The combined organic layers were washed with brine, dried over $\mathrm{MgSO}_{4}$ and the solvent was removed under reduced pressure. The crude product was purified as indicated.

\section{General procedure 2 (GP2): nitrogen cyclization}

Toluenesulfonic acid (0.15-0.20 equiv) was added to a solution of vinyl cyclopropyl ketone (0.02 $\mathrm{M}$ in anhydrous DCM). The reaction was stirred during the indicated time. The solution was quenched with $\mathrm{NaHCO}_{3}$ and extracted with $\mathrm{Et}_{2} \mathrm{O}$. The combined organic layers were washed with brine, dried over $\mathrm{MgSO}_{4}$ and the solvent was removed under reduced pressure. The cyclization products are purified as indicated and deprotected via GP4 for analysis (Cbz-protected products were difficult to analyze due to the presence of rotamers).

\section{General procedure 3 (GP3): homo-Nazarov cyclization}

A solution of Copper(II) triflate (0.15-0.20 equiv, $0.1 \mathrm{M}$ in anhydrous $\mathrm{CH}_{3} \mathrm{CN}$ ) was added dropwise to a solution of a vinyl cyclopropyl ketone derivative $\left(0.02 \mathrm{M}\right.$ in anhydrous $\left.\mathrm{CH}_{3} \mathrm{CN}\right)$. The reaction was stirred during the indicated time. The solution was quenched with $\mathrm{NaHCO}_{3}$ and extracted with $\mathrm{Et}_{2} \mathrm{O}$. The combined organic layers were washed with brine, dried over $\mathrm{MgSO}_{4}$ and the solvent was removed under reduced pressure. The cyclization products are purified as indicated and deprotected via GP4 for analysis (Cbzprotected products were difficult to analyze due to the presence of rotamers).

\section{General procedure 4 (GP4): hydrogenolysis}

To a solution $(0.02 \mathrm{M}$ in EtOH) of protected amine at room temperature $\mathrm{Pd} / \mathrm{C}$ (0.10 equiv) was added portionswise. Hydrogen gas was bubbled into the solution until the conversion of all starting material (controlled by TLC) ${ }^{[1]}$. The suspension was filtered on celite (pre-washed with DCM), washed with DCM and AcOEt and dried over $\mathrm{MgSO}_{4}$. The organic layer was evaporated on reduced pressure. No further purification was needed.

\section{General procedure 5 (GP5): Carboxylation of indoles}

Using a slight modification of a reported procedure, ${ }^{[2]}{ }^{n} \mathrm{BuLi}$ (2.5 $\mathrm{M}$ in pentane, 1.2 equiv) was added dropwise to a solution of substituted indole $\left(0.2 \mathrm{M}\right.$ in $\left.\mathrm{Et}_{2} \mathrm{O}\right)$ at $0^{\circ} \mathrm{C}$. The reaction was refluxed 2 hours then cooled to $0^{\circ} \mathrm{C}$ and $\mathrm{CO}_{2}$ was bubbled in the solution during 30 minutes. The suspension was quenched with

[1] A batch to batch dependency of the reaction time was observed (15 min to $5 \mathrm{~h}$ ). It is consequently important to monitor the reaction carefully via TLC.

[2] D. A Shirley, P. A. Roussel, J. Am. Chem. Soc. 1953, 75, 375. 
water and the organic layer was washed several times with water. The aqueous layer was acidified until $\mathrm{pH}$ $=2$ and the precipitate was filtered and dried under vacuum.

\section{Substrates synthesis}

\section{3,4-Dihydro-2H-pyridine-1-carboxylic acid benzyl ester (19)}

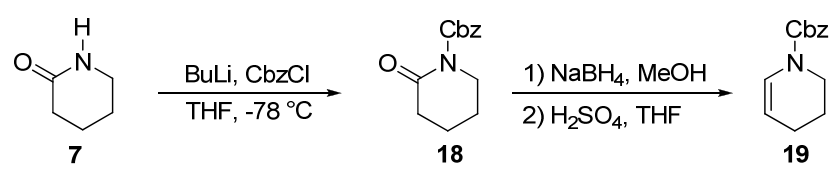

Following a reported procedure, ${ }^{[3]} \delta$-valerolactam (7) (3.00 g, $30.2 \mathrm{mmol}, 1.00$ equiv) was dissolved in THF $(130 \mathrm{~mL})$. The reaction mixture was cooled to $-78{ }^{\circ} \mathrm{C}$ and ${ }^{n} \mathrm{BuLi}(2.5 \mathrm{M}$ in hexane, $12 \mathrm{~mL}, 30 \mathrm{mmol}$, 1.0 equiv) was added dropwise to the resulting suspension. After $30 \mathrm{~min}$ at $-78{ }^{\circ} \mathrm{C}$, a solution of benzylchloroformate $(4.3 \mathrm{~mL}, 30 \mathrm{mmol}, 1.0$ equiv) in THF $(30 \mathrm{~mL})$ was added dropwise. After $4 \mathrm{~h}$ at -78 ${ }^{\circ} \mathrm{C}$, the reaction mixture was quenched with sat. $\mathrm{NH}_{4} \mathrm{Cl}(40 \mathrm{~mL})$ and warmed to $23{ }^{\circ} \mathrm{C}$. The reaction mixture was extracted with $\mathrm{Et}_{2} \mathrm{O}(3 \times 50 \mathrm{~mL})$. The combined organic layers were washed with brine $(50 \mathrm{~mL})$, dried over $\mathrm{MgSO}_{4}$ and the solvent was removed under reduced pressure to give the crude $\mathrm{Cbz}$ protected lactam 18 (6.95 g, $29.8 \mathrm{mmol}, 99 \%$ ), which was used without further purification.

$R_{f} 0.30$ (PET/AcOEt 2:1, Anisaldehyde);

${ }^{1} \mathrm{H}$ NMR $\left(\mathrm{CDCl}_{3}, 400 \mathrm{MHz}\right) \delta$ 7.47-7.28 (m, $\left.5 \mathrm{H} ; \mathrm{PhH}\right), 5.28\left(\mathrm{~s}, 2 \mathrm{H} ; \mathrm{OCH}_{2}\right), 3.74\left(\mathrm{~m}, 2 \mathrm{H} ; \mathrm{CH}_{2} \mathrm{~N}\right), 2.54$ (m, $\left.2 \mathrm{H} ; \mathrm{CH}_{2} \mathrm{CO}\right), 1.83$ (m, $4 \mathrm{H} ; \mathrm{CH}_{2}$ ).

Following a reported procedure ${ }^{[4]}$ protected lactame 18 (2.82 g, $12.1 \mathrm{mmol}, 1.00$ equiv) was dissolved in methanol $(52 \mathrm{~mL})$ at $0{ }^{\circ} \mathrm{C}$ and sodium borohydride $(0.46 \mathrm{~g}, 12 \mathrm{mmol}, 1.0$ equiv) was added portionswise. After the end of the addition, the reaction mixture was stirred at $0{ }^{\circ} \mathrm{C}$ for $15 \mathrm{~min}$ and poured onto ice-water $(150 \mathrm{~mL})$. The reaction mixture was extracted with AcOEt $(3 \times 100 \mathrm{~mL})$. The combined organic layers were washed with brine $(50 \mathrm{~mL})$, the combined water layers were extracted with AcOEt $(100 \mathrm{~mL})$ and the combined organic layers were dried over $\mathrm{MgSO}_{4}$ and the solvent was removed under reduced pressure.

The residues were dried in HV for $15 \mathrm{~min}$ and dissolved in THF $(25 \mathrm{~mL})$. Conc. sulfuric acid $(0.13 \mathrm{~mL})$ was added and the reaction was monitored via TLC (PET/AcOEt 10:1-2:1). After $1 \mathrm{~h}$ the reaction mixture was poured onto sat. $\mathrm{NaHCO}_{3}(100 \mathrm{~mL})$ and extracted with AcOEt $(3 \times 100 \mathrm{~mL})$. The combined organic layers were dried over $\mathrm{MgSO}_{4}$ and the solvent was removed under reduced pressure. The crude product was purified by flash column chromatography (PET/AcOEt 10:1-2:1) to yield enamide 19 (1.76 g, $8.11 \mathrm{mmol}$, $67 \%$ ) as a colorless oil.

[3] A. Giovannini, D. Savoia, A. Umani-Ronchi, J. Org. Chem. 1989, 54, 228.

[4] Y. Takeuchi, K. Azuma, M. Oshige, H. Abe, H. Nishioka, K. Sasaki, T. Harayama, Tetrahedron 2003, 59 , 1639. 
$R_{f} 0.80$ (PET/AcOEt 2:1, Anisaldehyde).

${ }^{1} \mathrm{H}$ NMR $\left(\mathrm{CDCl}_{3}, 400 \mathrm{MHz}\right) \delta$ 7.43-7.27 (m, $\left.5 \mathrm{H} ; \mathrm{PhH}\right), 6.89(\mathrm{~d}, J=8.6 \mathrm{~Hz}, 0.4 \mathrm{H}$; alkene H), $6.80(\mathrm{~d}, J$ $=8.6 \mathrm{~Hz}, 0.6 \mathrm{H}$; alkene H), $5.18\left(\mathrm{~s}, 2 \mathrm{H} ; \mathrm{CH}_{2} \mathrm{O}\right), 4.97(\mathrm{~m}, 0.4 \mathrm{H}$; alkene H), $4.86(\mathrm{~m}, 0.6 \mathrm{H}$; alkene H), 3.63 (m, $\left.2 \mathrm{H} ; \mathrm{CH}_{2} \mathrm{~N}\right), 2.04\left(\mathrm{~m}, 2 \mathrm{H} ; \mathrm{CH}_{2}\right), 1.83\left(\mathrm{~m}, 2 \mathrm{H} ; \mathrm{CH}_{2}\right)$.

${ }^{13} \mathrm{C} \mathrm{NMR}\left(\mathrm{CDCl}_{3}, 100 \mathrm{MHz}\right)$ (rotamers!) $\delta 151.7,136.3,128.5,128.5,128.2,128.0,128.0,125.3,124.8$, $106.7,106.4,67.4,67.3,42.3,42.2,21.6,21.4,21.2$.

IR v $2951(\mathrm{w}), 1703$ (s), 1653 (m), 1409 (s), 1347 (s), 1256 (s), 1227 (m), 1183 (w), 1108 (s), $1054(\mathrm{~m})$, $912(\mathrm{~m}), 764(\mathrm{~m}), 731(\mathrm{~s}), 697(\mathrm{~s})$.

${ }^{1} \mathrm{H}$ NMR corresponded to the literature values. ${ }^{[4]}$

2-Benzyl 7-ethyl 2-azabicyclo[4.1.0]heptane-2,7-dicarboxylate (20) and 2-(benzyloxycarbonyl)-2azabicyclo[4.1.0]heptane-7-carboxylic acid (21)

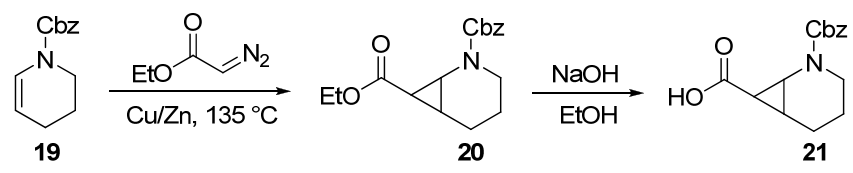

Following a reported procedure, ${ }^{[5]}$ a mixture of enamide 19 (1.70 g, $7.82 \mathrm{mmol}, 1.00$ equiv) and copper/bronze $(67 \mathrm{mg}$, prepared freshly as following: Zinc powder $(0.20 \mathrm{~g})$ was added to a solution of $\mathrm{CuSO}_{4} \cdot 5 \mathrm{H}_{2} \mathrm{O}(1.0 \mathrm{~g})$ in water $(2 \mathrm{~mL})$ over $10 \mathrm{~min}$ at RT. The resulting suspension was filtered, the solid was washed with water $(3 \times 5 \mathrm{~mL})$, ethanol $(3 \times 5 \mathrm{~mL})$ and $\mathrm{Et}_{2} \mathrm{O}(2 \times 5 \mathrm{~mL})$ and dried $1 \mathrm{~h}$ in $\left.\mathrm{HV}\right)$ was heated to 135 ${ }^{\circ} \mathrm{C}$. Ethyl diazoacetate $(4.2 \mathrm{~mL}, 40 \mathrm{mmol}, 5.0$ equiv) was added via syringe pump over $90 \mathrm{~min}$, and the reaction was stirred for further $30 \mathrm{~min}$ before cooling down to RT. The crude product was purified by two successive flash column chromatography (PET/AcOEt 10:1-2:1) to yield cyclopropane 20 (1.18 g, 3.89 $\mathrm{mmol}, 50 \%, \mathrm{R}_{\mathrm{f}}=0.30$ (PET/AcOEt 3:1), still containing some polymeric impurities) as a colorless oil.

The crude ester 20 (1.18 g, 3.89 mmol, 1.00 equiv) was dissolved in ethanol $(4 \mathrm{~mL})$ at $0{ }^{\circ} \mathrm{C}$ and $\mathrm{NaOH}$ ( $2.2 \mathrm{~g}, 55 \mathrm{mmol}, 14$ equiv) was added. The reaction mixture was stirred $1 \mathrm{~h}$ at $0{ }^{\circ} \mathrm{C}$ and $12 \mathrm{~h}$ at $\mathrm{RT}$, diluted with water $(10 \mathrm{~mL})$, acidified to $\mathrm{pH}=1$ with $1 \mathrm{M} \mathrm{HCl}$ and extracted with $\mathrm{CH}_{2} \mathrm{Cl}_{2}(3 \times 20 \mathrm{~mL})$. The combined organic layers were dried over $\mathrm{MgSO}_{4}$ and the solvent was removed under reduced pressure to give nearly pure acid 21 (0.89 g, $3.2 \mathrm{mmol}, 84 \%, 42 \%$ from enamide 19) as a colorless oil.

${ }^{1} \mathrm{H}$ NMR $\left(\mathrm{CDCl}_{3}, 400 \mathrm{MHz}\right) \delta$ 7.39-7.26 (m, $5 \mathrm{H}$; CH-Ar), 5.26-5.11 (m, $2 \mathrm{H} ; \mathrm{OCH}_{2}$ ), 3.88 (dt, $J=12.5$ $\mathrm{Hz}, 3.2 \mathrm{~Hz}, 0.7 \mathrm{H} ; \mathrm{NCH}_{2}$ or $\mathrm{NCH}$ rotamer A), 3.74 (s, $0.3 \mathrm{H} ; \mathrm{NCH}_{2}$ or $\mathrm{NCH}$ rotamer B), 3.57 (m, $0.3 \mathrm{H}$; $\mathrm{NCH}_{2}$ or $\mathrm{NCH}$ rotamer B), $3.50\left(\mathrm{dd}, J=8.6 \mathrm{~Hz}, 2.6 \mathrm{~Hz}, 0.7 \mathrm{H} ; \mathrm{NCH}_{2}\right.$ or $\mathrm{NCH}$ rotamer A), 2.77 (dd, $J=1.3$

[5] P. A. Grieco, M. D. Kaufman, J. Org. Chem. 1999, 64, 7586. 
$\mathrm{Hz}, 0.6 \mathrm{~Hz}, 0.3 \mathrm{H} ; \mathrm{NCH}_{2}$ or $\mathrm{NCH}$ rotamer B), 2.67 (td, $J=12.5 \mathrm{~Hz}, 2.2 \mathrm{~Hz}, 0.7 \mathrm{H} ; \mathrm{NCH}_{2}$ or $\mathrm{NCH}$ rotamer A), 2.08-1.78 (m, $3 \mathrm{H}$; $\mathrm{CHCO}$ and $\left.\mathrm{CH}_{2}\right), 1.77-1.58\left(\mathrm{~m}, 2 \mathrm{H}\right.$; $\mathrm{CH}$ or $\left.\mathrm{CH}_{2}\right), 1.40-1.20\left(\mathrm{~m}, 1 \mathrm{H}\right.$; $\mathrm{CH}$ or $\left.\mathrm{CH}_{2}\right)$.

${ }^{13} \mathrm{C} \mathrm{NMR}\left(\mathrm{CDCl}_{3}, 100 \mathrm{MHz}\right)$ (rotamers!) $\delta$ 178.0, 177.0, 156.4, 136.5, 136.3, 128.8, 128.6, 128.5, 128.3, $127.7,127.6,127.5,127.2,126.7,126.4,68.4,67.0,39.8,39.7,38.1,38.0,26.5,26.4,25.5,25.4,22.8,22.6$, 19.6, 19.4 .

IR v $3062(\mathrm{w}), 2946(\mathrm{w}), 2870(\mathrm{w}), 1689(\mathrm{~m}), 1448(\mathrm{w}), 1423(\mathrm{~m}), 1350(\mathrm{w}), 1302(\mathrm{w}), 1266(\mathrm{~m}), 1195$ (m), $1135(\mathrm{w}), 1098(\mathrm{w}), 1041(\mathrm{w}), 1002(\mathrm{w}), 909(\mathrm{w}), 731(\mathrm{~s}), 699(\mathrm{~s})$.

HRMS(ESI) calcd for $\mathrm{C}_{15} \mathrm{H}_{18} \mathrm{NO}_{4}{ }^{+}(\mathrm{M}+\mathrm{H}) 276.1236$, found 276.1232.

Benzyl 7-(methoxy(methyl)carbamoyl)-2-azabicyclo[4.1.0]heptane-2-carboxylate (22) and benzyl 7(1-methyl-1H-indole-2-carbonyl)-2-azabicyclo[4.1.0]heptane-2-carboxylate (4)

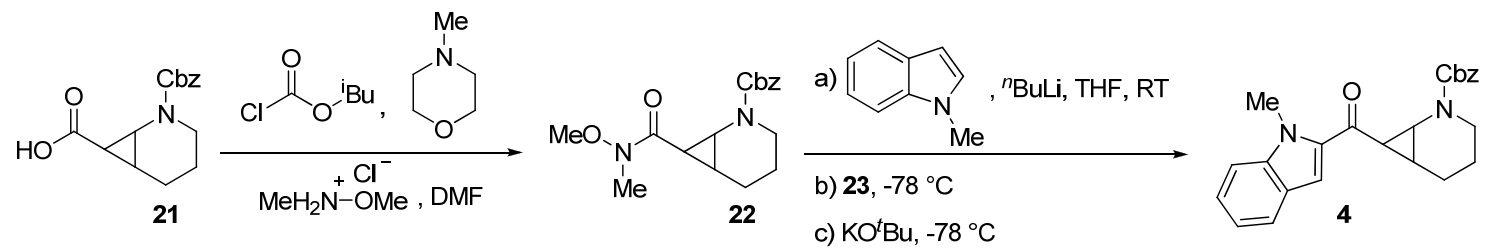

Following a reported procedure, ${ }^{[6]} \mathrm{N}$-methylmorpholine $(0.40 \mathrm{~mL}, 3.6 \mathrm{mmol}, 1.1$ equiv) was added to a solution of acid 21 (0.90 g, $3.3 \mathrm{mmol}, 1.0$ equiv) in DMF $(3.3 \mathrm{~mL})$ at $0{ }^{\circ} \mathrm{C}$. After $25 \mathrm{~min}$, isobutylchloroformate $\left(0.47 \mathrm{~mL}, 3.6 \mathrm{mmol}, 1.1\right.$ equiv) was added dropwise at $0{ }^{\circ} \mathrm{C}$. After $10 \mathrm{~min}, \mathrm{~N}, \mathrm{O}$ dimethylhydroxylamine hydrochloride $(0.35 \mathrm{~g}, 3.6 \mathrm{mmol}, 1.1$ equiv) was added, followed by $N$ methylmorpholine $\left(0.46 \mathrm{~mL}, 4.2 \mathrm{mmol}, 1.3\right.$ equiv) and the reaction mixture was warmed to $23{ }^{\circ} \mathrm{C}$. After 12 $\mathrm{h}$, the reaction was quenched with $0.5 \mathrm{M} \mathrm{HCl}(6 \mathrm{~mL})$ and extracted with $\mathrm{CH}_{2} \mathrm{Cl}_{2}(3 \times 10 \mathrm{~mL})$. The combined organic layers were washed with $0.5 \mathrm{M} \mathrm{NaOH}(2 \times 10 \mathrm{~mL})$, brine $(10 \mathrm{~mL})$, dried over $\mathrm{MgSO}_{4}$ and the solvent was removed under reduced pressure. The crude product was purified by flash column chromatography (PET/AcOEt 4:1-1:1) to yield Weinreb amide 22 (665 g, $2.09 \mathrm{mmol}, 64 \%, \mathrm{R}_{\mathrm{f}}=0.30$ (PET/AcOEt 1:1) as a colorless oil, which was used directly in the next step.

$N$-Methyl indole $\left(0.16 \mathrm{~mL}, 1.2 \mathrm{mmol}, 1.2\right.$ equiv) was diluted in THF $(4 \mathrm{~mL})$ at $0{ }^{\circ} \mathrm{C}$ and ${ }^{n} \mathrm{BuLi}(0.47 \mathrm{~mL}$, $1.2 \mathrm{mmol}, 1.2$ equiv) was added dropwise. The reaction mixture was stirred $1 \mathrm{~h}$ at RT and cooled to $-78{ }^{\circ} \mathrm{C}$. Weinreb amide 22 (dried through 3 co-evaporation with toluene, $0.31 \mathrm{~g}, 0.97 \mathrm{mmol}, 1.0$ equiv) was added dropwise via cannula as a cooled $\left(-78{ }^{\circ} \mathrm{C}\right)$ solution in THF $(2 \mathrm{~mL})$. After further stirring $2 \mathrm{~h}$ at $-78{ }^{\circ} \mathrm{C}$, a solution of $\mathrm{KO}^{t} \mathrm{Bu}(0.22 \mathrm{~g}, 2.0 \mathrm{mmol}, 2.0$ equiv) in THF $(2 \mathrm{~mL})$ was added. After $2 \mathrm{~h}$, the reaction mixture

[6] S. R. Nagarajan, H. F. Lu, A. F. Gasiecki, I. K. Khanna, M. D. Parikh, B. N. Desai, T. E. Rogers, M. Clare, B. B. Chen, M. A. Russell, J. L. Keene, T. Duffin, V. W. Engleman, M. B. Finn, S. K. Freeman, J. A. Klover, G. A. Nickols, M. A. Nickols, K. E. Shannon, C. A. Steininger, W. F. Westlin, M. M. Westlin, M. L. Williams, Bioorg. Med. Chem. 2007, 15, 3390. 
was warmed to $0{ }^{\circ} \mathrm{C}$, whereas the yellow suspension became an orange solution. After 5 min at $0{ }^{\circ} \mathrm{C}$, the reaction mixture was quenched with sat. $\mathrm{NaHCO}_{3}(10 \mathrm{~mL})$ and extracted with $\mathrm{Et}_{2} \mathrm{O}(3 \times 10 \mathrm{~mL})$. The combined organic layers were washed with brine $(10 \mathrm{~mL})$, dried over $\mathrm{MgSO}_{4}$ and the solvent was removed under reduced pressure. The crude product was purified by flash column chromatography (PET/AcOEt 5:13:1) to yield indole 4 (251 $\mathrm{mg}, 0.646 \mathrm{mmol}, 67 \%)$ as a colorless solid.

$\boldsymbol{R}_{\boldsymbol{f}} 0.30$ (PET/AcOEt 3:1, Anisaldehyde).

$\operatorname{Mp} 119-121^{\circ} \mathrm{C}$.

${ }^{1} \mathrm{H}$ NMR $\left(\mathrm{CDCl}_{3}, 400 \mathrm{MHz}\right) \delta 7.70$ (d, J=8.0 Hz, $1 \mathrm{H}$; CH-Ar), 7.43-7.28 (m, $4 \mathrm{H}$; CH-Ar), 7.22-7.14 (m, 1 H; CH-Ar), 7.10-7.02 (m, 2 H; CH-Ar), 6.99-6.89 (m, 2 H; CH-Ar), 5.16 (d, J=12.5 Hz, 1 H; OCH $)$, $4.99\left(\mathrm{~d}, J=12.5 \mathrm{~Hz}, 1 \mathrm{H} ; \mathrm{OCH}_{2}\right), 4.06\left(\mathrm{~s}, 0.8 \mathrm{H} ; \mathrm{CH}_{3}\right.$ rotamer B), 4.00 (s, $2.2 \mathrm{H} ; \mathrm{CH}_{3}$ rotamer A), 3.95 (t, $J$ $=3.8 \mathrm{~Hz}, 0.8 \mathrm{H} ; \mathrm{NCH}$ or $\mathrm{NCH}_{2}$ rotamer $\left.\mathrm{A}\right), 3.84\left(\mathrm{~m}, 0.2 \mathrm{H} ; \mathrm{NCH}\right.$ or $\left.\mathrm{NCH}_{2} \operatorname{rotamer} \mathrm{B}\right), 3.67(\mathrm{~m}, 0.2 \mathrm{H}$; $\mathrm{NCH}$ or $\mathrm{NCH}_{2}$ rotamer B), $3.55\left(\mathrm{dd}, J=8.3 \mathrm{~Hz}, 2.2 \mathrm{~Hz}, 0.8 \mathrm{H} ; \mathrm{NCH}\right.$ or $\left.\mathrm{NCH}_{2} \operatorname{rotamer} \mathrm{A}\right), 2.85$ (t, $J=12.5$ $\mathrm{Hz}, 0.2 \mathrm{H}$; $\mathrm{NCH}$ or $\mathrm{NCH}_{2}$ rotamer B), 2.76 (td, $J=12.5 \mathrm{~Hz}, 2.2 \mathrm{~Hz}, 0.8 \mathrm{H}$; $\mathrm{NCH}$ or $\mathrm{NCH}_{2}$ rotamer B), 2.63 (dd, $J=5.8 \mathrm{~Hz}, 2.2 \mathrm{~Hz}, 1 \mathrm{H} ; \mathrm{COCH}), 2.28$ (m, $1 \mathrm{H} ; \mathrm{CH}_{2} \mathrm{CH}$ ), 2.06-1.90 (m, $2 \mathrm{H} ; \mathrm{CH}_{2}$ ), 1.81-1.71 (m, $1 \mathrm{H}$; $\left.\mathrm{CH}_{2}\right), 1.48\left(\mathrm{~m}, 1 \mathrm{H} ; \mathrm{CH}_{2}\right)$.

${ }^{13} \mathrm{C} \mathrm{NMR}\left(\mathrm{CDCl}_{3}, 100 \mathrm{MHz}\right)$ (rotamers!) $\delta 189.9,156.4,140.0,136.2,135.4,128.4,128.0,127.5,127.5$, $126.8,125.9,125.7,125.5,122.8,122.6,120.6,120.3,111.3,111.1,110.2,67.1,65.1,41.6,41.0,33.5,32.8$, $32.0,29.6,23.8,21.8,19.9$.

IR v $2941(\mathrm{w}), 2866(\mathrm{w}), 1702$ (s), $1639(\mathrm{~s}), 1615(\mathrm{w}), 1513(\mathrm{~m}), 1428$ (s), 1407 (s), 1347 (s), 1299 (m), 1264 (m), 1195 (s), 1152 (m), 1129 (s), 1095 (m), 1034 (s), 908 (m), 794 (w), 769 (w), 752 (s), 731 (s), 698 (s), $648(\mathrm{w})$.

HRMS(ESI) calcd for $\mathrm{C}_{24} \mathrm{H}_{25} \mathrm{~N}_{2} \mathrm{O}_{3}{ }^{+}(\mathrm{M}+\mathrm{H}) 389.1865$, found 389.1854.

Benzyl 7-methyl-6-oxo-2,3,4,4a,5,6,7,11c-octahydro-1H-pyrido[3,2-c]carbazole-1-carboxylate (5) and 7-methyl-3,4,4a,5,7,11c-hexahydro-1H-pyrido[3,2-c]carbazol-6(2H)-one (6)
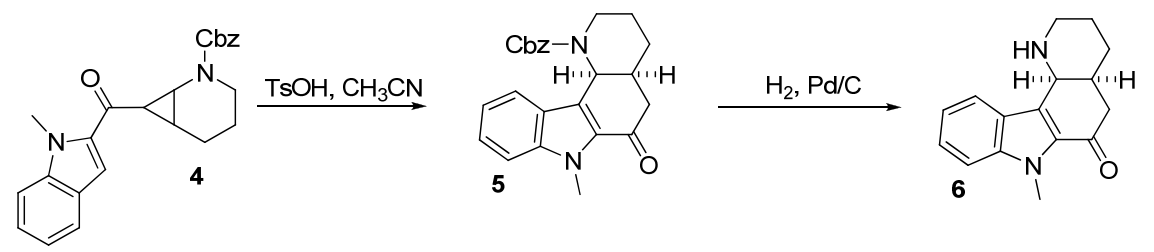
The reaction was performed following GP1, starting from cyclopropane 4 ( $50 \mathrm{mg}, 0.13 \mathrm{mmol}, 1.0$ equiv) and tosic acid ( $4.9 \mathrm{mg}, 0.030 \mathrm{mmol}, 0.20$ equiv). The reaction was quenched after $12 \mathrm{~h}$. Purification by flash chromatography (PET/AcOEt, 3:7) afforded 5 (45 mg, $0.12 \mathrm{mmol}, 90 \%$ ) as yellow oil. $R_{f} 0.60$ (PET/AcOEt 7:3, Anisaldehyde). The indole derivative $5(35 \mathrm{mg}, 0.090 \mathrm{mmol}, 1.0$ equiv) was deprotected following general procedure GP4, using $\mathrm{Pd} / \mathrm{C}(10 \mathrm{mg}, 10 \% \mathrm{w} / \mathrm{w})$ in $\mathrm{Et}_{2} \mathrm{O}(3.5 \mathrm{~mL})$ and a $\mathrm{H}_{2}$ balloon. The suspension was filtered on celite and washed with DCM to afford 6 as yellow oil in quantitative yield ( $23 \mathrm{mg}, 0.090$ mmol, 1.0 equiv).

$R_{f} 0.80\left(\mathrm{DCM} / \mathrm{MeOH} / \mathrm{Et}_{3} \mathrm{~N} 3: 1: 2 \%\right.$, Anisaldehyde).

${ }^{1} \mathrm{H}$ NMR $\left(\mathrm{CDCl}_{3}, 400 \mathrm{MHz}\right) 7.88\left(\mathrm{~d}, J=8.1 \mathrm{~Hz}, 1 \mathrm{H} ; \mathrm{H}_{\mathrm{m}}\right.$ or $\left.\mathrm{H}_{\mathrm{h}}\right), 7.43-7.31\left(\mathrm{~m}, 2 \mathrm{H} ; \mathrm{H}_{\mathrm{m}}\right.$ or $\mathrm{H}_{\mathrm{h}}$ and $\mathrm{H}_{\mathrm{i}}$ or $\left.\mathrm{H}_{\mathrm{l}}\right), 7.18\left(\mathrm{t}, J=7.4 \mathrm{~Hz}, 1 \mathrm{H} ; \mathrm{H}_{\mathrm{i}}\right.$ or $\left.\mathrm{H}_{\mathrm{l}}\right), 4.35\left(\mathrm{~d}, J=3.3 \mathrm{~Hz}, 1 \mathrm{H} ; \mathrm{H}_{\mathrm{f}}\right), 4.06\left(\mathrm{~s}, 3 \mathrm{H} ; 3 \mathrm{H}_{\mathrm{g}}\right), 3.23(\mathrm{dd}, J=16.5$ $\mathrm{Hz}, 12.0 \mathrm{~Hz}, 1 \mathrm{H} ; \mathrm{H}^{1}$ ) $) 2.96\left(\mathrm{~m}, 1 \mathrm{H} ; \mathrm{H}^{1}{ }_{\mathrm{a}}\right), 2.83\left(\mathrm{~m}, 1 \mathrm{H} ; \mathrm{H}^{2}{ }_{\mathrm{a}}\right), 2.52\left(\mathrm{~m}, 1 \mathrm{H} ; \mathrm{H}_{\mathrm{d}}\right), 2.41(\mathrm{dd}, J=16.5 \mathrm{~Hz}, 4.0$ $\mathrm{Hz}, 1 \mathrm{H} ; \mathrm{H}^{2}{ }_{\mathrm{e}}$ ), 1.87-1.60 ( $\mathrm{m}, 4 \mathrm{H} ; \mathrm{H}^{1}{ }_{\mathrm{c}}$ and/or $\mathrm{H}_{\mathrm{c}}{ }_{\mathrm{c}}$ and/or $\mathrm{H}_{\mathrm{b}}{ }_{\mathrm{b}}$ and/or $\mathrm{H}^{2}{ }_{\mathrm{b}}$ and $\left.\mathrm{NH}\right), 1.57-1.45\left(\mathrm{~m}, 1 \mathrm{H} ; \mathrm{H}_{\mathrm{b}}{ }_{\mathrm{b}}\right.$ or $\mathrm{H}_{\mathrm{b}}^{2}$ or $\mathrm{H}^{1}{ }_{\mathrm{c}}$ ).

${ }^{13} \mathrm{C} \mathrm{NMR}\left(\mathrm{CDCl}_{3}, 100 \mathrm{MHz}\right) \delta 192.2,139.7,130.2,126.6,123.9,121.4120 .7,110.3,110.3,51.4,45.3$, $41.0,35.9,31.5,29.7,28.5$.

IR v 3299 (w), 2926 (s), 2855 (m), 2149 (w), 1662 (s), 1616 (w), 1469 (m), 1432 (m), 1419 (w), 1386 (w), $1245(\mathrm{~m}), 1062(\mathrm{w}), 758(\mathrm{~s}), 746(\mathrm{~s}), 732(\mathrm{w}), 655(\mathrm{~m})$.

HRMS(ESI) calcd for $\mathrm{C}_{16} \mathrm{H}_{19} \mathrm{~N}_{2} \mathrm{O}^{+}(\mathrm{M}+\mathrm{H})$ 255.1497, found 255.1490.

Important correlations ROESY: $\mathrm{H}_{\mathrm{f}}-\mathrm{H}_{\mathrm{a}}{ }^{2} ; \mathrm{H}_{\mathrm{f}}-\mathrm{H}_{\mathrm{c}}{ }^{2} ; \mathrm{H}_{\mathrm{f}}-\mathrm{H}_{\mathrm{d}} ; \mathrm{H}_{\mathrm{f}}-\mathrm{H}_{\mathrm{e}}{ }^{2}$ (see the 2D spectra in section 6)

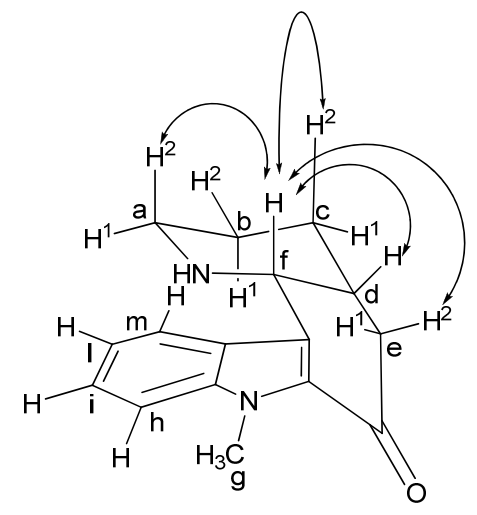

3,4-Dihydro-2H-3-ethylpyridine-1-carboxylic acid benzyl ester (8)

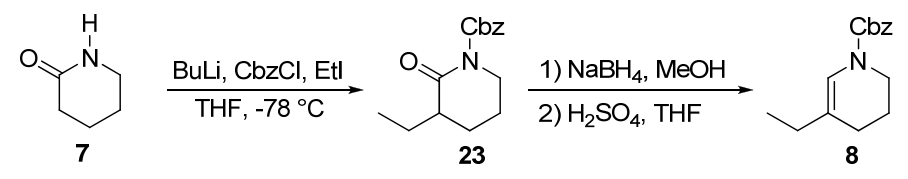


Following a reported procedure, ${ }^{[5]} \mathrm{a} 2.5 \mathrm{M}$ solution of ${ }^{n} \mathrm{BuLi}$ in pentane $(88.0 \mathrm{~mL}, 220 \mathrm{mmol} 2.20$ equiv) was added dropwise to a solution of $\delta$-valerolactam (7) (10.0 g, $100 \mathrm{mmol}, 1.00$ equiv) in THF (200 mL) at $0^{\circ} \mathrm{C}$. The reaction mixture was stirred during $30 \mathrm{~min}$ and distilled ethyl iodide $(12.2 \mathrm{~mL}, 150 \mathrm{mmol}, 1.50$ equiv) was added. The solution was stirred for additional 20 minutes before benzylchloroformate $(14.9 \mathrm{~mL}$, $105 \mathrm{mmol}, 1.05$ equiv) in THF $(50 \mathrm{~mL})$ was added. The reaction was stirred further 20 minutes, diluted with ether $(250 \mathrm{~mL})$ and washed with brine $(2 \times 50 \mathrm{~mL})$. The organic layer was dried over magnesium sulfate and concentrated under reduced pressure. The crude product was purified by flash column chromatography (PET/AcOEt 5:1) to yield lactame 23 (17.4 g, $66.5 \mathrm{mmol}, 67 \%)$ as colorless oil.

\section{$R_{f} 0.25$ (PET/AcOEt 5:1, Anisaldehyde).}

${ }^{1} \mathrm{H}$ NMR ( $\left.\mathrm{CDCl}_{3}, 400 \mathrm{MHz}\right) \delta 7.43$ (m, 2H; CH-Ar), 7.39-7.28 (m, $\left.3 \mathrm{H} ; \mathrm{CH}-\mathrm{Ar}\right), 5.27$ (s, $2 \mathrm{H} ; \mathrm{OCH}_{2}$ ), 3.81 (m, $\left.1 \mathrm{H} ; \mathrm{NCH}_{2}\right), 3.69$ (m, $1 \mathrm{H} ; \mathrm{NCH}_{2}$ ), 2.35 (m, $\left.1 \mathrm{H} ; \mathrm{CHCO}\right), 2.07-1.73$ (m, $4 \mathrm{H} ; \mathrm{CH}_{2} \mathrm{CH}_{2}$ ), 1.58-1.46 $\left(\mathrm{m}, 2 \mathrm{H} ; \mathrm{CH}_{2} \mathrm{CH}_{3}\right), 0.96\left(\mathrm{t}, J=7.5 \mathrm{~Hz}, 3 \mathrm{H} ; \mathrm{CH}_{3}\right)$.

Following a reported procedure, ${ }^{[4]}$ sodium borohydride $(829 \mathrm{mg}, 21.9 \mathrm{mmol}, 1.05$ equiv) was added portionswise into a solution of lactame $23\left(5.45 \mathrm{~g}, 20.9 \mathrm{mmol}, 1.00\right.$ equiv) in methanol $(100 \mathrm{~mL})$ at $0{ }^{\circ} \mathrm{C}$. After the end of the addition, the reaction mixture was stirred at $0{ }^{\circ} \mathrm{C}$ for $15 \mathrm{~min}$ and poured onto ice-water $(150 \mathrm{~mL})$. The reaction mixture was extracted with AcOEt $(3 \times 100 \mathrm{~mL})$. The combined organic layers were washed with brine $(50 \mathrm{~mL})$, the combined water layers were extracted with AcOEt $(100 \mathrm{~mL})$ and the combined organic layers were dried over $\mathrm{MgSO}_{4}$ and the solvent was removed under reduced pressure. The residue was dissolved in $\mathrm{Et}_{2} \mathrm{O}(50 \mathrm{~mL})$ and $0.5 \mathrm{~mL}$ of concentrated sulfuric acid was added dropwise. The reaction was stirred at RT for 1 hour then quenched with $\mathrm{K}_{2} \mathrm{CO}_{3}$ and dried on $\mathrm{Na}_{2} \mathrm{SO}_{4}$. The suspension was filtered and concentrated to afford 8 (4.75 g, $19.4 \mathrm{mmol}$, 93\% overall) without further purification as a colorless oil.

$R_{f} 0.36$ (PET/AcOEt 9:1, Anisaldehyde).

${ }^{1} \mathrm{H} \mathrm{NMR}\left(\mathrm{CDCl}_{3}, 400 \mathrm{MHz}\right) \delta$ 7.42-7.28 (m, $5 \mathrm{H}$; CH-Ar), 6.70 (s, $0.45 \mathrm{H}$; alkene-H rotamer A), 6.60 (s, $0.55 \mathrm{H}$; alkene-H rotamer B), 5.19 (s, $1.1 \mathrm{H} ; \mathrm{OCH}_{2}$ rotamer B), 5.17 (s, $0.9 \mathrm{H} ; \mathrm{OCH}_{2}$ rotamer A), 3.57 (m, 2 $\mathrm{H} ; \mathrm{NCH}_{2}$ ), 2.07-1.94 (m, $4 \mathrm{H}$; allylic $\left.\mathrm{CH}_{2}\right), 1.82$ (m, $\left.2 \mathrm{H} ; \mathrm{CH}_{2} \mathrm{CH}_{2}\right), 1.06-0.97$ (m, $\left.3 \mathrm{H} ; \mathrm{CH}_{3}\right)$.

${ }^{13} \mathrm{C} \mathrm{NMR}\left(\mathrm{CDCl}_{3}, 100 \mathrm{MHz}\right)$ (rotamers!) $\delta 153.4,153.0,136.5,136.4,128.3,127.9,127.8,121.0,120.6$, 119.1, 118.7, 67.1, 67.0, 41.9, 41.8, 28.1, 28.1, 24.8, 24.7, 21.6, 21.6, 12.6, 12.4 .

IR $v 2962$ (w), 2934 (w), 2880 (w), 1703 (s), 1499 (w), 1409 (s), 1345 (m), 1313 (m), 1258 (s), 1202 (m), 1176 (m), $1111(\mathrm{~m}), 1041$ (m), 988 (m), 914 (m), 882 (m), 761 (m), 738 (m), 698 (m), 635 (m), 607 (m). 
HRMS(ESI) calcd for $\mathrm{C}_{15} \mathrm{H}_{20} \mathrm{NO}_{2}^{+}(\mathrm{M}+\mathrm{H})$ 246.1494, found 246.1496.

1-Benzyloxycarbonyl-5-ethyl-1-azabicyclo[4.1.0]heptan-7-carboxylic acid ethyl ester (9) 1Benzyloxycarbonyl-5-ethyl-1-azabicyclo[4.1.0]heptan-7-carboxylic acid (10)

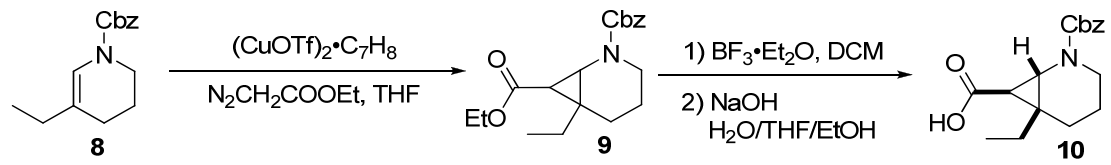

Following a slight modification of a reported procedure, ${ }^{[7]}$ a solution of ethyl diazoacetate $(6.2 \mathrm{~mL}, 59$ mmol, 4.0 equiv) in DCM (15 mL) was added to a solution of enamine 8 (3.64 g, $14.9 \mathrm{mmol}, 1.00$ equiv) and copper triflate (I) toluene complex (192 mg, $0.370 \mathrm{mmol}, 0.0200$ equiv) in DCM (15mL) over $18 \mathrm{~h}(1.3$ $\mathrm{mL} / \mathrm{h}$ ) via syringe pump. After the addition was complete, the reaction was concentrated and purified by flash column chromatography (PET/AcOEt 15:1 until PET/AcOEt 9:1) to yield 9 (3.74 g, $11.3 \mathrm{mmol}, 76 \%$ ) as colorless oil. Following a reported procedure, ${ }^{[5]}$ the mixture of exo and endo esters $(1.50 \mathrm{~g}, 4.53 \mathrm{mmol}$, 1.00 equiv) in dichlorometane $(20 \mathrm{~mL})$ at $-20^{\circ} \mathrm{C}$ was treated with $\mathrm{BF}_{3} \cdot \mathrm{Et}_{2} \mathrm{O}(96 \mathrm{mg}, 0.68 \mathrm{mmol}, 0.15$ equiv $)$. The reaction was allowed to warm at $0^{\circ} \mathrm{C}$ and stirred at the same temperature until the isomerization was finished (from $R_{f}$ 0.28-0.32 PET/AcOEt 9:1 to $R_{f}$ 0.30 PET/AcOEt 9:1, Anisaldehyde). Triethylamine (1 $\mathrm{mL})$ was added dropwise to the reaction and the mixture was diluted with $\mathrm{Et}_{2} \mathrm{O}(50 \mathrm{~mL})$ and washed with water and brine. The organic layer was dried over $\mathrm{Na}_{2} \mathrm{SO}_{4}$ and evaporated under reduced pressure to give a pale yellow oil $(1.50 \mathrm{~g})$ which was used directly in the next step.

The crude oil (1.50 g, $4.53 \mathrm{mmol}, 1.00$ equiv) was dissolved in a solution of water/THF/EtOH 1/1/3 (25 $\mathrm{mL}$ total) at $0^{\circ} \mathrm{C}$ and $\mathrm{NaOH}(1.63 \mathrm{~g}, 40.3 \mathrm{mmol}, 9.00$ equiv) was added portionswise. The reaction was heated to $60^{\circ} \mathrm{C}$ and stirred during 2 hours. The solution was concentrated, then diluted with water $(30 \mathrm{~mL})$ and washed with $\mathrm{Et}_{2} \mathrm{O}(3 \times 20 \mathrm{~mL})$. The aqueous layer was acidified with $\mathrm{HCl}(1 \mathrm{M}$ aqueous solution) until pH 2 and extracted with DCM $(3 \times 20 \mathrm{~mL})$ to give $\mathbf{1 0}$ as a colorless oil which turns solid upon storage (1.25 g, $4.12 \mathrm{mmol}, 91 \%$ overall). No further purification was needed.

${ }^{1} \mathrm{H}$ NMR $\left(\mathrm{CDCl}_{3}, 400 \mathrm{MHz}\right) \delta$ 7.39-7.21 (m, $5 \mathrm{H}$; CH-Ar), 5.23-5.09 (m, $\left.2 \mathrm{H} ; \mathrm{OCH}_{2}\right), 3.86(\mathrm{dt}, J=12.5$ $\mathrm{Hz}, 3.1 \mathrm{~Hz}, 0.7 \mathrm{H} ; \mathrm{NCH}_{2}$ rotamer A), 3.73 (m, $0.3 \mathrm{H} ; \mathrm{NCH}_{2}$ rotamer B), 3.55 (d, J=3.5 Hz, 0.3 H; $\mathrm{NCH}$ rotamer B), 3.49 (d, $J=3.6 \mathrm{~Hz}, 0.7 \mathrm{H}$; $\mathrm{NCH}$ rotamer A), 2.77 (dt, $J=12.5 \mathrm{~Hz}, 1.7 \mathrm{~Hz}, 0.3 \mathrm{H}$; $\mathrm{NCH}_{2}$ rotamer B), $2.66\left(\mathrm{dt}, J=12.5 \mathrm{~Hz}, 3.4 \mathrm{~Hz}, 0.7 \mathrm{H} ; \mathrm{NCH}_{2}\right.$ rotamer A), 2.05 (m, $1 \mathrm{H}$; CHCO), 1.93-1.12 (m, $\left.6 \mathrm{H} ; \mathrm{CH}_{2}\right)$, 0.99 (t, $J=7.2 \mathrm{~Hz}, 1 \mathrm{H} ; \mathrm{CH}_{3}$ rotamer B), 0.93 (t, $J=7.4 \mathrm{~Hz}, 2 \mathrm{H} ; \mathrm{CH}_{3}$ rotamer A).

[7] R. Beumer, C. Bubert, C. Cabrele, O. Vielhauer, M. Pietzsch, O. Reiser, J. Org. Chem. 2000, 65, 8960. 
${ }^{13} \mathrm{C} \mathrm{NMR}\left(\mathrm{CDCl}_{3}, 100 \mathrm{MHz}\right)$ (rotamers!) $\delta 177.2,176.9,156.2,136.5,128.5,128.4,128.1,127.7,127.2$, $67.1,67.0,44.9,44.8,41.5,41.1,34.7,34.0,31.1,30.6,25.9,25.7,21.5,21.3,20.9,10.3,9.9$.

IR v 2956 (w), 2939 (w), 2864 (w), 1704 (s), 1584 (w), 1456 (s), 1423 (s), 1348 (m), 1270 (m), 1240 (m), 1214 (s), 1129 (m), 1036 (m), 1017 (w), 948 (w), 909 (m), 883 (m), 752 (m), 736 (s), 698 (m), 676 (m), $668(\mathrm{~m}), 635(\mathrm{~m})$.

HRMS(ESI) calcd for $\mathrm{C}_{17} \mathrm{H}_{22} \mathrm{NO}_{4}{ }^{+}(\mathrm{M}+\mathrm{H}) 304.1549$, found 304.1548 .

\section{$N$-Methoxy- $N$-methylcarbamo-6-yl-5-ethyl-1-azabicyclo[4.1.0]heptanes-1-benzylcarboxylate (11)}

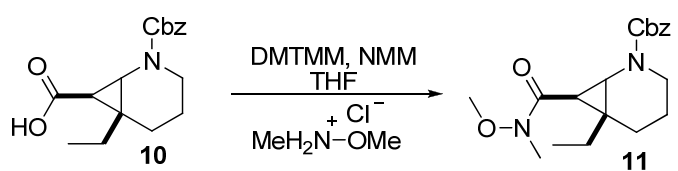

Dimethoxytriazin- $N$-methylmorpholinium chloride ${ }^{[8]}$ (DMTMM, $900 \mathrm{mg}, 3.24 \mathrm{mmol}, 1.50$ equiv) was suspended into a solution of acid $10(655 \mathrm{mg}, 2.16 \mathrm{mmol}, 1.00$ equiv) in THF $(7.5 \mathrm{~mL})$ and the reaction mixture was stirred at RT during $60 \mathrm{~min}$. $\mathrm{N}, \mathrm{O}$-dimethylhydroxylamine hydrochloride $(97.5 \mathrm{mg}, 2.16 \mathrm{mmol}$, 1.00 equiv) was added, followed by $N$-methylmorpholine ( $475 \mu \mathrm{L}, 4.32 \mathrm{mmol}, 2.00$ equiv) and the reaction mixture was stirred during 36 hours. The reaction was quenched with a 5\% aqueous solution of citric acid and extracted with $\mathrm{Et}_{2} \mathrm{O}$. The combined organic layers were dried over $\mathrm{MgSO}_{4}$ and the solvent was removed under reduced pressure. The crude reaction mixture was purified by flash column chromatography (AcOEt/PET 3:7) to afford 11 (695 mg, $2.00 \mathrm{mmol}$, 93\%) as colorless oil.

$R_{f} 0.30$ (PET/AcOEt 7:3, Anisaldehyde).

${ }^{1} \mathrm{H}$ NMR $\left(\mathrm{CDCl}_{3}, 400 \mathrm{MHz}\right) \delta$ 7.35-7.19 (m, $5 \mathrm{H}$; CH-Ar), $5.23\left(\mathrm{~d}, J=12.9 \mathrm{~Hz}, 0.8 \mathrm{H} ; \mathrm{OCH}_{2}\right.$ rotamer A), $5.14\left(\mathrm{~d}, J=12.5 \mathrm{~Hz}, 0.2 \mathrm{H} ; \mathrm{OCH}_{2} \operatorname{rotamer~B}\right), 5.07$ (d, $\left.J=13.0 \mathrm{~Hz}, 1 \mathrm{H} ; \mathrm{OCH}_{2}\right), 3.84(\mathrm{dt}, J=12.5 \mathrm{~Hz}$, $3.4 \mathrm{~Hz}, 0.8 \mathrm{H} ; \mathrm{NCH}_{2}$ rotamer A), $3.71\left(\mathrm{~m}, 0.2 \mathrm{H} ; \mathrm{NCH}_{2}\right.$, rotamer B), 3.64 (s, 0.6 H; $\mathrm{OCH}_{3}$ rotamer B), 3.58$3.52\left(\mathrm{~m}, 2.4 \mathrm{H} ; \mathrm{OCH}_{3}\right.$ rotamer A and $\mathrm{NCH}$ rotamer B), 3.49 (d, $J=3.7 \mathrm{~Hz}, 0.8 \mathrm{H}$; NCH rotamer A), 3.15 (s, $0.6 \mathrm{H} ; \mathrm{NCH}_{3}$ rotamer B), $3.12\left(\mathrm{~s}, 2.4 \mathrm{H} ; \mathrm{NCH}_{3} \operatorname{rotamer} \mathrm{A}\right), 2.73\left(\mathrm{t}, J=11.7 \mathrm{~Hz}, 0.2 \mathrm{H} ; \mathrm{NCH}_{2}\right.$ rotamer B), $2.64\left(\mathrm{dt}, J=12.5 \mathrm{~Hz}, 2.2 \mathrm{~Hz}, 0.8 \mathrm{H} ; \mathrm{NCH}_{2}\right.$ rotamer A), 2.09-1.87 (m, $2 \mathrm{H} ; \mathrm{CHCO}$ and $\left.\mathrm{CH}_{2}\right), 1.77-1.54(\mathrm{~m}$, $\left.4 \mathrm{H} ; \mathrm{CH}_{2}\right), 1.36$ (m, $\left.1 \mathrm{H} ; \mathrm{CH}_{2}\right), 0.85$ (t, $\left.J=7.4 \mathrm{~Hz}, 3 \mathrm{H} ; \mathrm{CH}_{3}\right)$.

[8] M. Kunishima, C. Kawachi, F. Iwasaki, K. Terao, S. Tani, Tetrahedron Letters 1999, 40, 5327. 
${ }^{13} \mathrm{C} \mathrm{NMR}\left(\mathrm{CDCl}_{3}, 100 \mathrm{MHz}\right) \delta$ (rotamers!) 171.2, 156.2, 136.8, 128.2, 128.1, 127.8, 127.8, 127.4, 127.0, $66.8,66.5,61.2,42.9,42.7,41.5,41.0,33.0,32.4,29.1,28.5,26.0,25.9,25.2,21.6,10.3$.

IR v 2964 (w), 2937 (w), 2876 (w), 1703 (s), 1651 (s), 1458 (m), 1417 (s), 1384 (m), 1358 (m), 1344 (m) (m), 1295 (m), 1266 (m), 1209 (m), 1180 (m), 1123 (m), 1016 (m), 913 (m), 769 (w), 734 (s), 700 (w).

HRMS(ESI) calcd for $\mathrm{C}_{19} \mathrm{H}_{27} \mathrm{~N}_{2} \mathrm{O}_{4}{ }^{+}(\mathrm{M}+\mathrm{H}) 347.1971$, found 347.1978.

\section{6-(1-Methyl-1H-indol-2-carbonyl)-5-ethyl-1-azabicyclo[4.1.0]heptane-1-benzylcarboxylate (12)}

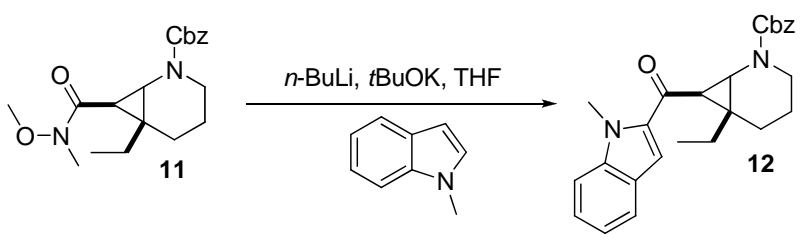

A solution of $N$-methylindole ( $13 \mu \mathrm{L}, 0.10 \mathrm{mmol}, 1.3$ equiv) in THF $(0.5 \mathrm{~mL})$ was stirred at $0^{\circ} \mathrm{C}$. Then a solution of ${ }^{n} \mathrm{BuLi}(2.5 \mathrm{M}$ in pentane, $38 \mu \mathrm{L}, 0.10 \mathrm{mmol}, 1.2$ equiv) diluted in $\mathrm{THF}(0.2 \mathrm{~mL})$ was added dropwise. The solution was warmed to RT, stirred for 1 hour and then cooled to $-78^{\circ} \mathrm{C}$. From a separate flask, a solution of Weinreb amide $11(27 \mathrm{mg}, 0.080 \mathrm{mmol}, 1.0$ equiv) in THF (0.4 mL) was added via cannula into the solution. The mixture was stirred 2 hours at $-78^{\circ} \mathrm{C}$, then a solution of ${ }^{t} \mathrm{BuOK}$ in $\mathrm{THF}(0.3$ $\mathrm{mL}$ ) was added dropwise. The reaction was stirred 2 hours at $-78^{\circ} \mathrm{C}$ and $5 \mathrm{~min}$ at $0^{\circ} \mathrm{C}$, quenched with $\mathrm{NaHCO}_{3}$ and extracted with $\mathrm{Et}_{2} \mathrm{O}(5 \times 3 \mathrm{~mL})$. The organic layers were dried over $\mathrm{Na}_{2} \mathrm{SO}_{4}$, evaporated on reduced pressure and purified on flash chromatography (AcOEt/PET 1:5) to give 12 (16 mg, $0.038 \mathrm{mmol}$ $48 \%$ ) as a yellow oil.

$R_{f} 0.60$ (PET/AcOEt 7:3, Anisaldehyde).

${ }^{1} \mathrm{H}$ NMR $\left(\mathrm{CDCl}_{3}, 400 \mathrm{MHz}\right) \delta 7.70$ (d, $J=8.1 \mathrm{~Hz}, 1 \mathrm{H}$; CH-Ar), 7.45-7.29 (m, $4 \mathrm{H}$; CH-Ar), 7.25-7.00 (m, $5 \mathrm{H}$; CH-Ar), 5.19 (d, $\left.J=12.7 \mathrm{~Hz}, 1 \mathrm{H} ; \mathrm{OCH}_{2}\right), 5.04$ (d, $\left.J=12.7 \mathrm{~Hz}, 1 \mathrm{H} ; \mathrm{OCH}_{2}\right), 4.08$ (s, $0.6 \mathrm{H} ; \mathrm{CH}_{3}$ rotamer B), 3.97 (s, $2.4 \mathrm{H} ; \mathrm{CH}_{3}$ rotamer A), 3.95 (dt, $J=13.2 \mathrm{~Hz}, 3.4 \mathrm{~Hz}, 0.8 \mathrm{H} ; \mathrm{NCH}_{2}$, rotamer A), 3.88$3.82\left(\mathrm{~m}, 0.2 \mathrm{H} ; \mathrm{NCH}_{2}\right.$, rotamer B), 3.77 (d, $\left.J=3.4 \mathrm{~Hz}, 1 \mathrm{H} ; \mathrm{NCH}\right), 2.83$ (t, $J=11.9 \mathrm{~Hz}, 0.2 \mathrm{H} ; \mathrm{NCH}_{2}$, rotamer B), $2.74\left(\mathrm{dt}, J=12.0 \mathrm{~Hz}, 1.9 \mathrm{~Hz}, 0.8 \mathrm{H} ; \mathrm{NCH}_{2}\right.$, rotamer A), $2.68(\mathrm{~d}, J=3.1 \mathrm{~Hz}, 0.2 \mathrm{H}$; $\mathrm{COCH}$; rotamer B), $2.65\left(\mathrm{~d}, J=3.6 \mathrm{~Hz}, 0.8 \mathrm{H}\right.$; $\mathrm{COCH}$; rotamer A), $2.18\left(\mathrm{~m}, 1 \mathrm{H} ; \mathrm{CH}_{2}\right), 1.90-1.48\left(\mathrm{~m}, 5 \mathrm{H} ; \mathrm{CH}_{2}\right)$, $0.85\left(\mathrm{t}, J=7.4 \mathrm{~Hz}, 3 \mathrm{H} ; \mathrm{CH}_{3}\right)$. 
${ }^{13} \mathrm{C} \mathrm{NMR}\left(\mathrm{CDCl}_{3}, 100 \mathrm{MHz}\right)$ (rotamers!) $\delta 190.8,157.3,140.9,137.6,137.5,129.4,129.1,128.5,128.2$, $126.9,126.6,126.3,123.7,121.7,111.7,111.3,67.9,46.2,45.9,42.3,42.6,39.2,38.8,38.4,33.0,27.3$, $27.0,26.0,23.0,22.811 .7,11.5$.

IR v 3064 (w), 3033 (w), 2938 (w), 2876 (w), 1703 (s), 1646 (s), 1614 (w), 1512 (m), 1464 (s), 1428 (s), 1408 (s), 1348 (s), 1268 (m), 1211 (s), 1196 (s), 1163 (m), 1129 (m), 1049 (m), 1027 (m), 910 (m), 769 (m), $737(\mathrm{~s}), 698(\mathrm{~m})$.

HRMS(ESI) calcd for $\mathrm{C}_{26} \mathrm{H}_{29} \mathrm{~N}_{2} \mathrm{O}_{3}{ }^{+}(\mathrm{M}+\mathrm{H})$ 417.2178, found 417.2181.

Benzyl 4a-ethyl-7-methyl-6-oxo-2,3,4,4a,5,6,7,11c-octahydro-1H-pyrido[3,2-c]carbazole-1carboxylate (24) and 4a-ethyl-7-methyl-3,4,4a,5,7,11c-hexahydro-1H-pyrido[3,2-c]carbazol-6(2H)-one (13)
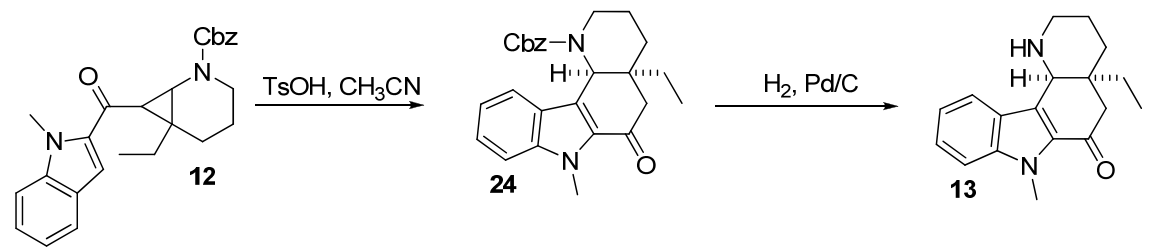

The reaction was performed following general procedure GP1, starting from cyclopropane 12 (14 mg, 0.034 mmol, 1.0 equiv) and tosic acid ( $1 \mathrm{mg}, 7 \mu \mathrm{mol}, 0.2$ equiv). The reaction was quenched after $5 \mathrm{~min}$ to give 24 (14 mg, $0.034 \mathrm{mmol}$, quant) without further purification as yellow oil $\left(R_{f} 0.65\right.$ (PET/AcOEt 7:3, Anisaldehyde)). The indole derivative 24 (14 mg, $34 \mu \mathrm{mol}, 1.0$ equiv) was deprotected following general procedure GP2, using $\mathrm{Pd} / \mathrm{C}(3 \mathrm{mg} 10 \% \mathrm{w} / \mathrm{w})$ in $\mathrm{Et}_{2} \mathrm{O}(1.5 \mathrm{~mL})$ and $\mathrm{H}_{2}$ balloon. The suspension was filtered on celite and washed with DCM to afford 13 as green oil in quantitative yield (9.6 mg, $34 \mu \mathrm{mol})$.

$R_{f} 0.75\left(\mathrm{DCM} / \mathrm{MeOH} / \mathrm{Et}_{3} \mathrm{~N}\right.$ 3:1:2\%, Anisaldehyde).

${ }^{1} \mathrm{H}$ NMR $\left(\mathrm{CDCl}_{3}, 400 \mathrm{MHz}\right) \delta 8.01$ (d, $\left.J=8.2 \mathrm{~Hz}, 1 \mathrm{H} ; \mathrm{CH}-\mathrm{Ar}\right), 7.45-7.32$ (m, $2 \mathrm{H}$; CH-Ar), 7.22 (t, $J=$ $7.2 \mathrm{~Hz}, 1 \mathrm{H}$; CH-Ar), 4.16 (s, $1 \mathrm{H} ; \mathrm{CHN}), 4.08$ (s, $\left.3 \mathrm{H} ; \mathrm{NCH}_{3}\right), 3.53$ (d, J=17.0 Hz, $\left.1 \mathrm{H} ; \mathrm{CH}_{2} \mathrm{CO}\right), 2.52$ (m, $\left.J=14.6 \mathrm{~Hz}, 2 \mathrm{H} ; \mathrm{CH}_{2} \mathrm{~N}\right), 2.26\left(\mathrm{~d}, J=16.8 \mathrm{~Hz}, 1 \mathrm{H} ; \mathrm{CH}_{2} \mathrm{CO}\right), 1.87-1.21\left(\mathrm{~m}, 7 \mathrm{H} ; \mathrm{CH}_{2}\right.$ and $\left.\mathrm{NH}\right), 0.75(\mathrm{t}, J=$ $\left.7.4 \mathrm{~Hz}, 3 \mathrm{H} ; \mathrm{CH}_{3}\right)$.

${ }^{13} \mathrm{C} \mathrm{NMR}\left(\mathrm{CDCl}_{3}, 100 \mathrm{MHz}\right) \delta 191.6,139.8,129.9,126.6,124.3,121.5,120.8,110.3,110.3,55.6,45.0$, $44.0,40.2,32.6,31.6,31.2,29.7,27.5$.

IR v 3300 (w), 2960 (m), 2935 (m), 2902 (w), 1702 (s), 1662 (s), 1615 (w), 1601 (w), 1542 (w), 1448 (m), 1411 (m), 1314 (m), 1252 (m), 1179 (w), 1117 (w), 1070 (w), 751 (m), 698 (m), 641 (m), 627 (m), 607 (m). 
HRMS(ESI) calcd for $\mathrm{C}_{18} \mathrm{H}_{23} \mathrm{~N}_{2} \mathrm{O}^{+}(\mathrm{M}+\mathrm{H})$ 283.1810, found 283.1813.

\section{6-(1H-indol-2-carbonyl)-5-ethyl-1-azabicyclo[4.1.0]heptane-1-benzylcarboxylate (1a)}

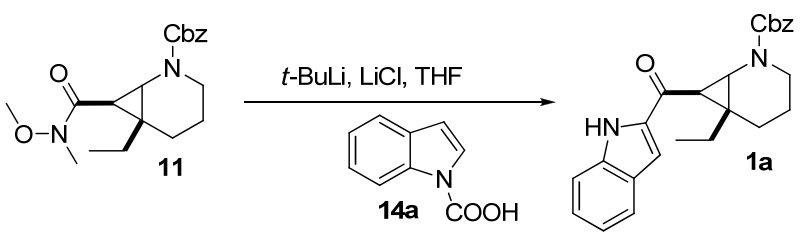

${ }^{t} \mathrm{BuLi}(1.6 \mathrm{M}$ in pentane, $38 \mu \mathrm{L}, 0.80 \mathrm{mmol}, 2.2$ equiv) was added dropwise into a solution of carboxylindole 14a prepared following GP5 (64 mg, $0.40 \mathrm{mmol}, 1.1$ equiv) and $\mathrm{LiCl}^{[9]}$ (17 mg, $0.40 \mathrm{mmol}$, 1.1 equiv) in THF $(1 \mathrm{~mL})$ at $-78^{\circ} \mathrm{C}$. The solution was stirred for 3 hours and then transferred via cannula into a solution of amide $11\left(125 \mathrm{mg}, 360 \mu \mathrm{mol}, 1.00\right.$ equiv) in THF $(1 \mathrm{~mL})$ at $-78^{\circ} \mathrm{C}$. The reaction was allowed to warm to $-20^{\circ} \mathrm{C}$ over 5 hours then transferred via cannula into saturated aqueous $\mathrm{NaHCO}_{3}$ solution $(10 \mathrm{~mL})$ at $0^{\circ} \mathrm{C}$. The aqueous phase was extracted with $\mathrm{Et}_{2} \mathrm{O}(5 \times 10 \mathrm{~mL})$. The organic layers were dried over $\mathrm{Na}_{2} \mathrm{SO}_{4}$, evaporated under reduced pressure and purified on flash chromatography with deactivated silica (AcOEt/PET 1:9) to give 1a (97 mg, $24 \mathrm{mmol}, 67 \%$ ) as yellow oil.

$R_{f} 0.70$ (PET/AcOEt 7:3, Anisaldehyde).

${ }^{1} \mathrm{H}$ NMR $\left(\mathrm{CDCl}_{3}, 400 \mathrm{MHz}\right) \delta 9.39(\mathrm{~d}, J=0.4 \mathrm{~Hz}, 0.2 \mathrm{H}$; NH rotamer B), $9.29(\mathrm{~m}, 0.8 \mathrm{H}$; NH rotamer A), 7.72 (d, $J=8.0 \mathrm{~Hz}, 1 \mathrm{H}$; CH-Ar), 7.45 (m, $1 \mathrm{H}$; CH-Ar), 7.36 (m, $2 \mathrm{H}$; CH-Ar), 7.16 (m, 4 H; CH-Ar), 7.02 (m, $2 \mathrm{H}$; CH-Ar), 5.21-5.08 (m, $0.4 \mathrm{H} ; \mathrm{OCH}_{2}$ rotamer B), 5.15 (d, $\left.J=12.7 \mathrm{~Hz}, 0.8 \mathrm{H} ; \mathrm{OCH}_{2} \operatorname{rotamer} \mathrm{A}\right)$ $5.02\left(\mathrm{~d}, J=12.7 \mathrm{~Hz}, 0.8 \mathrm{H} ; \mathrm{OCH}_{2} \operatorname{rotamer} \mathrm{A}\right), 3.93\left(\mathrm{~m}, 1 \mathrm{H} ; \mathrm{NCH}_{2}\right), 3.81(\mathrm{~d}, J=3.3 \mathrm{~Hz}, 1 \mathrm{H} ; \mathrm{NCH}), 2.84(\mathrm{t}$, $\left.J=12.2 \mathrm{~Hz}, 0.2 \mathrm{H} ; \mathrm{NCH}_{2} \operatorname{rotamer} \mathrm{B}\right), 2.75\left(\mathrm{t}, J=12.1 \mathrm{~Hz}, 0.8 \mathrm{H} ; \mathrm{NCH}_{2} \operatorname{rotamer} \mathrm{A}\right), 2.61(\mathrm{~d}, J=3.3 \mathrm{~Hz}, 1$ $\mathrm{H} ; \mathrm{COCH}), 2.15$ (m, $\left.1 \mathrm{H} ; \mathrm{CH}_{2}\right), 1.77\left(\mathrm{~m}, 4 \mathrm{H} ; \mathrm{CH}_{2}\right), 1.54\left(\mathrm{~m}, 1 \mathrm{H} ; \mathrm{CH}_{2}\right), 0.91\left(\mathrm{~m}, 0.6 \mathrm{H} ; \mathrm{CH}_{3}\right.$ rotamer B), $0.82\left(\mathrm{t}, J=7.3 \mathrm{~Hz}, 2.4 \mathrm{H} ; \mathrm{CH}_{3}\right.$ rotamer A).

${ }^{13} \mathrm{C} \mathrm{NMR}\left(\mathrm{CDCl}_{3}, 100 \mathrm{MHz}\right)$ (rotamers!) $\delta$ 188.6, 156.3, 137.1, 136.6, 136.5, 128.5, 128.1, 128.1, 127.7, $127.5,127.3$, 126.1, 122.9, 122.7, 120.9, 120.7, 112.1, 108.6, 108.4, 66.9, 46.0, 45.6, 41.7, 41.3, 38.3, 36.7, $36.0,26.3,26.1,25.0,21.9,21.8,10.7,10.6$.

IR v $3309(\mathrm{w}), 2960$ (w), 2936 (w), 2876 (w), 1700 (s), 1629 (s), 1521 (m), 1446 (m), 1409 (s), 1349 (s), 1313 (w), 1299 (w), 1268 (m), 1232 (w), 1210 (m), 1192 (m), 1164 (m), 1140 (s), 1080 (w), 1034 (w), 1010 (w), $978(w), 911(\mathrm{~m}), 799(\mathrm{w}), 746(\mathrm{~s}), 736(\mathrm{~s}), 698(\mathrm{~m}), 606(\mathrm{w})$.

[9] $\mathrm{LiCl}$ was dried under $\mathrm{HV}(<0.05 \mathrm{Torr})$, warmed at $600^{\circ} \mathrm{C}(\mathrm{Mp})$ and stirred. It was then cooled down to RT and dissolved into $1 \mathrm{~mL}$ of dry THF. 
HRMS(ESI) calcd for $\mathrm{C}_{25} \mathrm{H}_{27} \mathrm{~N}_{2} \mathrm{O}_{3}{ }^{+}(\mathrm{M}+\mathrm{H})$ 403.2022, found 403.2034.

Benzyl 4a-ethyl-6-oxo-2,3,4,4a,5,6,7,11c-octahydro-1H-pyrido[3,2-c]carbazole-1-carboxylate (15a); 4a-ethyl-7-3,4,4a,5,7,11c-hexahydro-1 $\mathrm{H}$-pyrido[3,2-c]carbazol-6(2H)-one (25a); benzyl 4a-ethyl-6-oxo2,3,4,4a,5,6-hexahydroindolo[1,2-a][1,8]naphthyridine-1(12aH)-carboxylate (16a); 4a-ethyl-

\section{1,2,3,4,4a,5-hexahydroindolo[1,2-a][1,8]naphthyridin-6(12aH)-one (26a)}
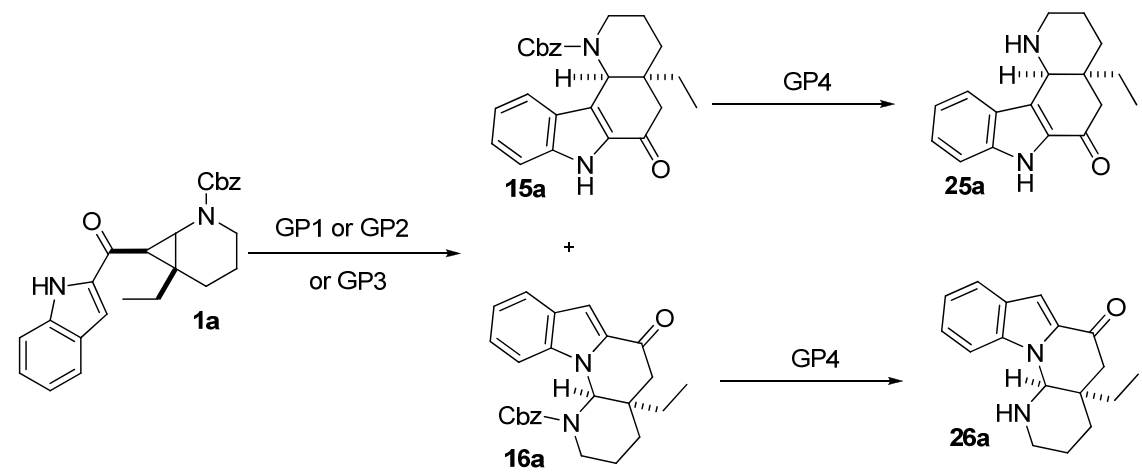

The reaction was performed following general procedure GP1, starting from cyclopropane 1a (35 mg, $0.087 \mathrm{mmol}, 1.0$ equiv) and tosic acid ( $3.0 \mathrm{mg}, 16 \mu \mathrm{mol}, 0.20$ equiv). The reaction was quenched after 15 min and the crude oil was purified by column chromatography (PET/AcOEt 9:1, Anisaldehyde) to give the product 15a $(R f \quad 0.65,16 \mathrm{mg}, 39 \mu \mathrm{mol}, 45 \%)$ and the product 16a $(R f 0.75,10 \mathrm{mg}, 25 \mu \mathrm{mol}, 29 \%)$. The tetracyclic compound 15a (16 mg, $16 \mu \mathrm{mol}, 1.0$ equiv) was deprotected following the general procedure GP4 to give 25a (10 mg, $38 \mu \mathrm{mol}, 97 \%)$ as a yellow oil without further purification. Product 16a (10 mg, $25 \mu \mathrm{mol}, 1.0$ equiv) was deprotected following the general procedure GP4 to give 26a $(6.7 \mathrm{mg}, 25 \mu \mathrm{mol}, 99$ $\%)$ as a yellow oil without further purification.

The reaction was performed using general procedure GP2, starting from cyclopropane 1a (100 mg, 0.250 mmol, 1.00 equiv) and copper (II) triflate ( $9.0 \mathrm{mg}, 25 \mu \mathrm{mol}, 0.10$ equiv). The reaction was quenched after 15 min and the crude oil was purified by column chromatography (PET/AcOEt 7:3, Anisaldehyde) to give the product 15a $(R f \quad 0.65,80 \mathrm{mg}, 0.20 \mathrm{mmol}, 80 \%)$ and the product 16a $(R f 0.75,11 \mathrm{mg}, 30 \mu \mathrm{mol}, 11 \%)$. The tetracyclic compound 15a (70 $\mathrm{mg}, 0.17 \mathrm{mmol}, 1.0$ equiv) was deprotected following the general procedure GP4 to give 25a (45 mg, $0.17 \mathrm{mmol}$, quant.) as a yellow oil without further purification.

The reaction was performed following general procedure GP3, starting from cyclopropane $1 \mathrm{a}(0.10 \mathrm{~g}, 0.25$ mmol, 1.0 equiv) and tosic acid ( $4.7 \mathrm{mg}, 25 \mu \mathrm{mol}, 0.1$ equiv). The reaction was quenched after $15 \mathrm{~min}$ and the crude oil was purified by column chromatography (PET/AcOEt 7:3, Anisaldehyde) to give the product 15a $(R f 0.65,4.0 \mathrm{mg}, 10 \mu \mathrm{mol}, 4 \%)$ and the product 16a $(R f 0.75,85 \mathrm{mg}, 0.22 \mathrm{mmol}, 85 \%)$. The tetracyclic compound 16a (50 mg, $0.12 \mathrm{mmol}, 1.0$ equiv) was deprotected following the general procedure GP4 to give 26a (33 mg, $0.12 \mathrm{mmol}$, quant.) as a yellow oil without further purification. 
$R_{f} 0.0$ (AcOEt/PET 6:4, Anisaldehyde).

${ }^{1} \mathrm{H}$ NMR (CD $\left.\mathrm{OD}, 400 \mathrm{MHz}\right) \delta 7.91$ (d, $J=8.2 \mathrm{~Hz}, 1 \mathrm{H}$; CH-Ar), 7.51 (d, $J=8.4 \mathrm{~Hz}, 1 \mathrm{H}$; CH-Ar), 7.40 (d, $J=7.2 \mathrm{~Hz}, 1 \mathrm{H}$; CH-Ar), 7.23 (t, $J=7.5 \mathrm{~Hz}, 1 \mathrm{H} ; \mathrm{CH}-\mathrm{Ar}), 4.61(\mathrm{~s}, 1 \mathrm{H} ; \mathrm{CHN}), 3.40-3.11$ (m, $3 \mathrm{H} ; \mathrm{CH}_{2} \mathrm{~N}$ and $\mathrm{CH}_{2} \mathrm{CO}$ ), 2.37 (d, $\left.J=17.6 \mathrm{~Hz}, 1 \mathrm{H} ; \mathrm{CH}_{2} \mathrm{CO}\right), 2.16(\mathrm{~s}, 1 \mathrm{H} ; \mathrm{NH}), 1.92\left(\mathrm{~m}, 2 \mathrm{H} ; \mathrm{CH}_{2}\right), 1.80(\mathrm{~m}, 2 \mathrm{H}$; $\left.\mathrm{CH}_{2}\right), 1.45\left(\mathrm{~m}, 2 \mathrm{H} ; \mathrm{CH}_{2}\right), 0.83\left(\mathrm{t}, J=7.5 \mathrm{~Hz}, 3 \mathrm{H} ; \mathrm{CH}_{3}\right)$.

${ }^{13} \mathrm{C}$ NMR $\left(\mathrm{CD}_{3} \mathrm{OD}, 100 \mathrm{MHz}\right) \delta 191.2,140.0,132.6,128.2,126.1,122.4,122.0,121.6,114.1,55.6$, $45.3,42.7,41.5,33.0,32.7,20.4,7.8$.

IR v 2961 (s), 2943 (s), 2866 (s), 2142 (m), 1660 (w), 1543 (w), 1493 (m), 1464 (m), 1366 (w), 1263 (w), 1167 (m), 1105 (m), 1073 (m), 1021 (m), 884 (m), 804 (m), 751 (m), 673 (s), 635 (s).

HRMS(ESI) calcd for $\mathrm{C}_{17} \mathrm{H}_{21} \mathrm{~N}_{2} \mathrm{O}^{+}(\mathrm{M}+\mathrm{H})$ 269.1654, found 269.1655 .

In order to obtain a crystalline compound, a solution of 25a was treated with an excess of $\mathrm{HCl}$ (4 M in dioxane). The solvents were removed under reduced pressure and the colorless solid obtained was recrystallized by slow diffusion of hexane into a solution in dichloromethane. For X-ray image see the spectra in section 6 (CCDC number: 777832).

26a

$R_{f}$ 0.4 (AcOEt/PET 3:7 Anisaldehyde).

${ }^{1} \mathrm{H}$ NMR $\left(\mathrm{CD}_{3} \mathrm{OD}, 400 \mathrm{MHz}\right) \delta 7.71\left(\mathrm{~d}, J=8.1 \mathrm{~Hz}, 1 \mathrm{H} ; \mathrm{H}_{\mathrm{h}}\right), 7.63\left(\mathrm{~d}, J=8.4 \mathrm{~Hz}, 1 \mathrm{H} ; \mathrm{H}_{\mathrm{m}}\right), 7.43(\mathrm{t}, J=$ $7.9 \mathrm{~Hz}, 1 \mathrm{H} ; \mathrm{H}_{\mathrm{i}}$ or $\left.\mathrm{H}_{\mathrm{l}}\right), 7.28\left(\mathrm{~s}, 1 \mathrm{H} ; \mathrm{H}_{\mathrm{g}}\right), 7.18\left(\mathrm{t}, J=7.7 \mathrm{~Hz}, 1 \mathrm{H} ; \mathrm{H}_{\mathrm{i}}\right.$ or $\left.\mathrm{H}_{1}\right), 5.22\left(\mathrm{~s}, 1 \mathrm{H} ; \mathrm{H}_{\mathrm{f}}\right), 3.36(\mathrm{~d}, J=$ $\left.17.6 \mathrm{~Hz}, 1 \mathrm{H} ; \mathrm{H}_{\mathrm{e}}{ }^{2}\right), 3.12\left(\mathrm{~m}, 1 \mathrm{H} ; \mathrm{H}_{\mathrm{a}}{ }^{2}\right), 2.97\left(\mathrm{~m}, 1 \mathrm{H} ; \mathrm{H}_{\mathrm{a}}{ }^{1}\right), 2.32\left(\mathrm{~d}, J=17.6 \mathrm{~Hz}, 1 \mathrm{H} ; \mathrm{H}_{\mathrm{e}}{ }^{1}\right), 1.87-1.60(\mathrm{~m}, 5 \mathrm{H}$; $\mathrm{H}_{\mathrm{b}}{ }^{1} ; \mathrm{H}_{\mathrm{b}}{ }^{2} ; \mathrm{H}_{\mathrm{c}}{ }^{1} ; \mathrm{H}_{\mathrm{c}}{ }^{2}$ and $\left.\mathrm{NH}\right), 1.31\left(\mathrm{q}, J=7.6 \mathrm{~Hz}, 2 \mathrm{H} ; 2 \mathrm{H}_{\mathrm{n}}\right), 0.80\left(\mathrm{t}, J=7.5 \mathrm{~Hz}, 3 \mathrm{H} ; 3 \mathrm{H}_{\mathrm{o}}\right)$.

${ }^{13} \mathrm{C}$ NMR $\left(\mathrm{CD}_{3} \mathrm{OD}, 100 \mathrm{MHz}\right) \delta 192.9,138.5,133.1,128.6,127.5,124.3,122.8,111.7,107.4,70.7$, $45.9,41.4,40.2,33.6,31.6,22.1,7.4$.

IR v 2934 (w), 2861 (w), 1676 (s), 1532 (s), 1446 (w), 1407 (w), 1360 (w), 1322 (m), 1247 (w), 1196 (w), $1173(\mathrm{w}), 1146(\mathrm{w}), 1119(\mathrm{w}), 1044(\mathrm{w}), 929(\mathrm{w}), 894(\mathrm{w}), 872(\mathrm{w}), 846(\mathrm{w}), 811(\mathrm{w}), 740(\mathrm{~m}), 634$ (w).

HRMS(ESI) calcd for $\mathrm{C}_{17} \mathrm{H}_{21} \mathrm{~N}_{2} \mathrm{O}^{+}(\mathrm{M}+\mathrm{H}) 269.1654$, found 269.1652.

Important correlations NOESY: $\mathrm{H}_{\mathrm{f}}-\mathrm{H}_{\mathrm{a}}{ }^{1} ; \mathrm{H}_{\mathrm{f}}-2 \mathrm{H}_{\mathrm{n}} ; \mathrm{H}_{\mathrm{f}}-3 \mathrm{H}_{\mathrm{o}} ; \mathrm{H}_{\mathrm{f}}-\mathrm{H}_{\mathrm{e}}{ }^{1} ; \mathrm{H}_{\mathrm{f}}-\mathrm{H}_{\mathrm{m}}$ (see the $2 \mathrm{D}$ spectra in section 6) 


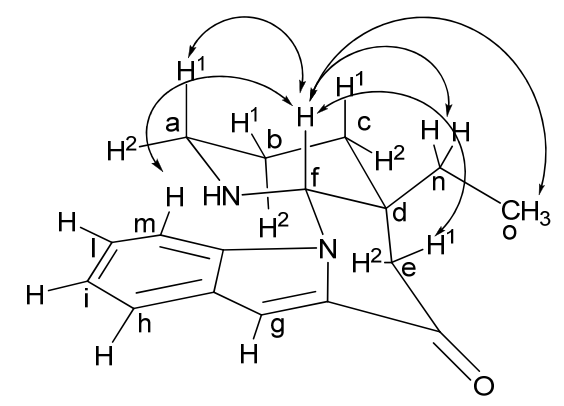

6-(5-methoxy-1H-indol-2-carbonyl)-5-ethyl-1-azabicyclo[4.1.0]heptane-1-benzylcarboxylate (1b)

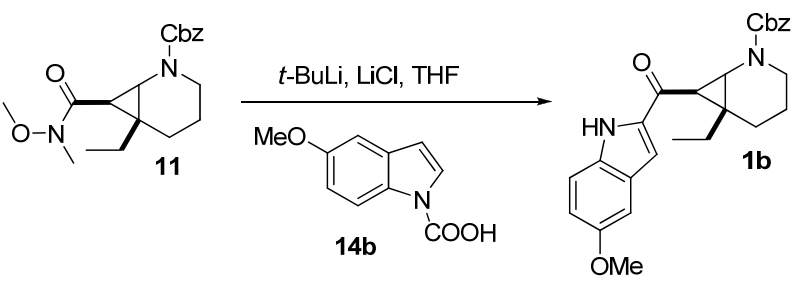

${ }^{t} \mathrm{BuLi}$ (1.6 M in pentane, $0.65 \mathrm{~mL}, 1.0 \mathrm{mmol}, 2.6$ equiv) was added dropwise into a solution of 5-metoxycarboxylindole 14b (100 mg, $0.520 \mathrm{mmol}, 1.30$ equiv) prepared following GP5 and $\mathrm{LiCl}^{[9]}$ (44.0 mg, 1.04 mmol, 2.60 equiv) in THF $(3.5 \mathrm{~mL})$ at $-78^{\circ} \mathrm{C}$. The solution was stirred over 3 hours then transferred via cannula into a solution of amide 11 (139 mg, $0.400 \mathrm{mmol}, 1.00$ equiv) in THF $(3.5 \mathrm{~mL})$ at $0^{\circ} \mathrm{C}$ over 20 minutes. The reaction was stirred at $0^{\circ} \mathrm{C}$ over 20 minutes then transferred via cannula into a saturated aqueous solution of $\mathrm{NaHCO}_{3}(10 \mathrm{~mL})$ at $0^{\circ} \mathrm{C}$. The aqueous layer was extracted with $\mathrm{Et}_{2} \mathrm{O}(5 \times 10 \mathrm{~mL})$. The organic layers were dried over $\mathrm{Na}_{2} \mathrm{SO}_{4}$, evaporated under reduced pressure and purified on flash chromatography with deactivated silica (AcOEt/toluene 1:9) to give $\mathbf{1 b}(99.8 \mathrm{mg}, 23.0 \mathrm{mmol}, 58 \%$ ) as a yellow oil with tendency to solidify and amide 11 (53 mg, $0.15 \mathrm{mmol}, 38 \%$ ).

$R_{f} 0.25$ (AcOEt/toluene 9:1, Anisaldehyde).

${ }^{1} \mathrm{H}$ NMR $\left(\mathrm{CDCl}_{3}, 400 \mathrm{MHz}\right) \delta 9.82(\mathrm{~s}, 0.8 \mathrm{H}$; NH rotamer A), 9.74 (s, 0.2 H; NH rotamer B), 7.58-6.92 (m, $9 \mathrm{H}$; CH-Ar), 5.32-5.10 (m, $0.4 \mathrm{H} ; \mathrm{OCH}_{2}$ rotamer B), 5.17 (d, $J=12.8 \mathrm{~Hz}, 0.8 \mathrm{H}$; $\mathrm{OCH}_{2}$ rotamer A), $5.03\left(\mathrm{~d}, J=12.6 \mathrm{~Hz}, 0.8 \mathrm{H} ; \mathrm{OCH}_{2}\right.$ rotamer A), $3.98\left(\mathrm{~m}, 1 \mathrm{H} ; \mathrm{NCH}_{2}\right), 3.92-3.83\left(\mathrm{~m}, 4 \mathrm{H} ; \mathrm{OCH}_{3}\right.$ and $\left.\mathrm{NCH}\right)$, 2.87 (t, $J=11.5 \mathrm{~Hz}, 0.2 \mathrm{H} ; \mathrm{NCH}_{2}$ rotamer B), 2.78 (t, $\left.J=11.9 \mathrm{~Hz}, 0.8 \mathrm{H} ; \mathrm{NCH}_{2} \operatorname{rotamer} \mathrm{A}\right), 2.64(\mathrm{~d}, J=3.2$ $\mathrm{Hz}, 1 \mathrm{H} ; \mathrm{COCH}), 2.17$ (m, $\left.1 \mathrm{H} ; \mathrm{CH}_{2}\right), 1.94-1.73$ (m, $\left.4 \mathrm{H} ; \mathrm{CH}_{2}\right), 1.58\left(\mathrm{~m}, 1 \mathrm{H} ; \mathrm{CH}_{2}\right), 0.85$ (t, J=6.9 Hz, $3 \mathrm{H}$; $\left.\mathrm{CH}_{3}\right)$

${ }^{13} \mathrm{C} \mathrm{NMR}\left(\mathrm{CDCl}_{3}, 100 \mathrm{MHz}\right)$ (rotamers!) $\delta 188.6,156.3,154.6,137.1,136.4,132.9,128.4,128.2,128.0$, $127.9,127.4,127.2,118.0,117.7,117.4,112.9,112.9,109.7,108.3,108.0,102.9,67.2,66.9,55.6,45.9$, $45.3,41.7,41.2,38.1,36.7,36.0,26.3,26.0,25.0,21.9,10.7$. 
IR v $3303(\mathrm{w}), 2941(\mathrm{w}), 2866(\mathrm{w}), 1778(\mathrm{w}), 1703(\mathrm{~m}), 1626(\mathrm{~m}), 1525(\mathrm{~s}), 1458$ (m), $1414(\mathrm{~m}), 1352$ (m), 1296 (w), 1269 (w), 1211 (s), 1171 (m), 1124 (m), 1029 (w), $911(w), 742(\mathrm{~m}), 677$ (w), $630(\mathrm{w})$.

HRMS(ESI) calcd for $\mathrm{C}_{26} \mathrm{H}_{29} \mathrm{~N}_{2} \mathrm{O}_{4}{ }^{+}(\mathrm{M}+\mathrm{H})$ 433.2127, found 433.2113 .

Benzyl ethyl-10-methoxy-6-oxo-2,3,4,4a,5,6,7,11c-octahydro-1H-pyrido[3,2-c]carbazole-1-carboxylate (15b); ethyl-10-methoxy-3,4,4a,5,7,11c-hexahydro-1H-pyrido[3,2-c]carbazol-6(2H)-one (25b); benzyl 4a-ethyl-9-methoxy-6-0xo-2,3,4,4a,5,6-hexahydroindolo[1,2-a][1,8]naphthyridine-1(12aH) carboxylate (16b) and 4a ethyl-9-methoxy-1,2,3,4,4a,5-hexahydroindolo[1,2-a][1,8]naphthyridin-6(12aH)-one (26b)

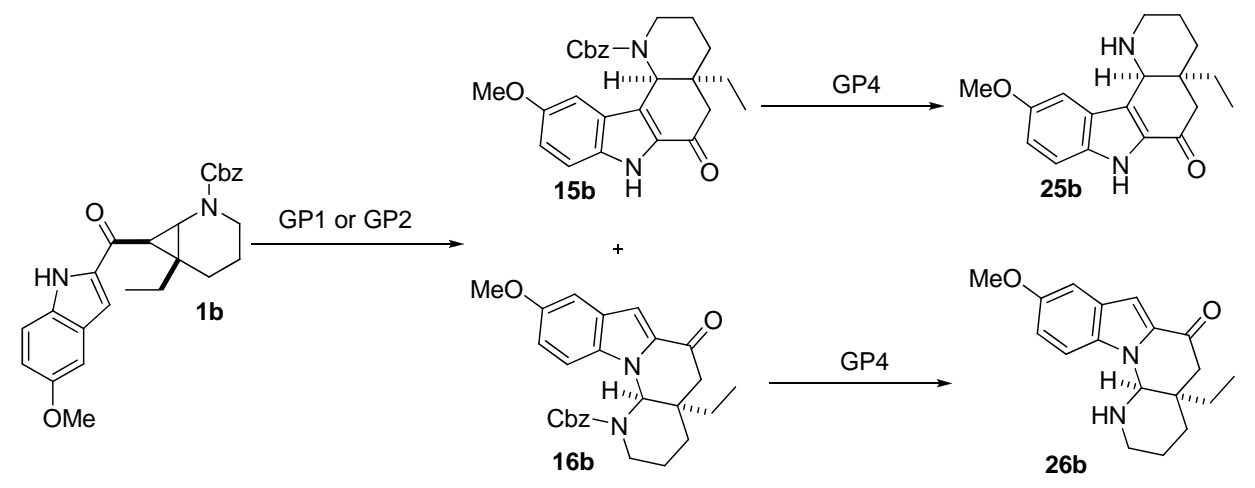

The reaction was performed using general procedure GP2, starting from cyclopropane $\mathbf{1 b}$ (74 $\mathrm{mg}, 0.17$ mmol, 1.0 equiv) and copper (II) triflate (9.2 mg, $25 \mu \mathrm{mol}, 0.15$ equiv). The reaction was quenched after 15 min and the crude oil was purified by column chromatography (PET/AcOEt 8:2, Anisaldehyde) to give the product 15b $(R f 0.3,62 \mathrm{mg}, 0.14 \mathrm{mmol}, 84 \%)$ and the product 16b ( $R f 0.4,8.0 \mathrm{mg}, 80 \mu \mathrm{mol}, 11 \%)$. The tetracyclic compound 15b (50 mg, $0.12 \mathrm{mmol}, 1.0$ equiv) was deprotected following the general procedure GP4 to give 25b (35 mg, $0.12 \mathrm{mmol}$, quant.) as a yellow oil without further purification.

The reaction was performed following general procedure GP3, starting from cyclopropane $1 \mathbf{b}$ (67 mg, 0.16 mmol, 1.0 equiv) and tosic acid ( $4.0 \mathrm{mg}, 23 \mu \mathrm{mol}, 0.15$ equiv). The reaction was quenched after $15 \mathrm{~min}$ and the crude oil was purified by column chromatography (PET/AcOEt 8:2, Anisaldehyde) to give the product 15b $(R f 0.3,3 \mathrm{mg}, 7 \mu \mathrm{mol}, 4 \%)$ and the product $\mathbf{1 6 b}(R f 0.4,55 \mathrm{mg}, 0.13 \mathrm{mmol}, 82 \%)$. The tetracyclic compound 16b (53 mg, $0.12 \mathrm{mmol}, 1.0$ equiv) was deprotected following the general procedure GP4 to give 26b (36 mg, $0.12 \mathrm{mmol}$, quant.) as a yellow oil without further purification.

25b

$R_{f} 0.0$ (AcOEt/PET 6:4 Anisaldehyde).

${ }^{1} \mathrm{H}$ NMR $\left(\mathrm{CDCl}_{3}, 400 \mathrm{MHz}\right) \delta 9.29$ (s, $1 \mathrm{H}$; NH indole), 7.30 (d, J=9.0 Hz, $\left.1 \mathrm{H} ; \mathrm{CH}-\mathrm{Ar}\right), 7.15$ (s, $1 \mathrm{H}$; CH-Ar), 7.01 (dd, $J=8.9 \mathrm{~Hz}, 1.9 \mathrm{~Hz}, 1 \mathrm{H}$; CH-Ar), 4.01 (s, $1 \mathrm{H} ; \mathrm{CHN}), 3.86$ (s, $\left.3 \mathrm{H} ; \mathrm{OCH}_{3}\right), 3.16$ (d, $J=$ 
$16.8 \mathrm{~Hz}, 1 \mathrm{H} ; \mathrm{CH}_{2} \mathrm{CO}$ ), 3.06 (m, $\left.1 \mathrm{H} ; \mathrm{CH}_{2} \mathrm{~N}\right), 2.83$ (m, $\left.1 \mathrm{H} ; \mathrm{CH}_{2} \mathrm{~N}\right), 2.27$ (d, J=16.8 Hz, $1 \mathrm{H} ; \mathrm{CH}_{2} \mathrm{CO}$ ), 1.92 (br s, $1 \mathrm{H}$; NH amine), 1.79-1.62 (m, $\left.2 \mathrm{H} ; \mathrm{CH}_{2}\right), 1.52$ (m, $\left.2 \mathrm{H} ; \mathrm{CH}_{2}\right), 1.42-1.17$ (m, $\left.2 \mathrm{H} ; \mathrm{CH}_{2}\right), 0.81$ (t, $J=$ $\left.7.5 \mathrm{~Hz}, 3 \mathrm{H} ; \mathrm{CH}_{3}\right)$.

${ }^{13} \mathrm{C} \mathrm{NMR}\left(\mathrm{CDCl}_{3}, 100 \mathrm{MHz}\right) \delta 191.4,154.7,133.6,131.0,127.5,125.9,118.5,113.6,100.9,56.4,55.7$, $45.4,42.8,40.7,33.0,31.0,22.7,7.7$.

IR v $3281(\mathrm{w}), 2936(\mathrm{w}), 2857$ (w), 1652 (s), $1540(\mathrm{w}), 1487$ (m), 1464 (w), 1439 (w), 1335 (w), 1297 (w), $1264(\mathrm{~m}), 1218(\mathrm{~s}), 1169(\mathrm{~m}), 1121(\mathrm{w}), 1105(\mathrm{w}), 1030(\mathrm{w}), 909(\mathrm{~m}), 809(\mathrm{w}), 733(\mathrm{~s}), 628(\mathrm{w})$.

HRMS(ESI) calcd for $\mathrm{C}_{18} \mathrm{H}_{23} \mathrm{~N}_{2} \mathrm{O}^{+}(\mathrm{M}+\mathrm{H}) 299.1760$, found 299.1755.

\section{6b}

$R_{f}$ 0.2 (AcOEt/PET 4:6 Anisaldehyde).

${ }^{1} \mathrm{H}$ NMR $\left(\mathrm{CDCl}_{3}, 400 \mathrm{MHz}\right) \delta 7.31$ (d, $J=9.0 \mathrm{~Hz}, 1 \mathrm{H}$; CH-Ar), 7.19 (s, $\left.1 \mathrm{H} ; \mathrm{CH}-\mathrm{Ar}\right), 7.10$ (d, $J=2.0$

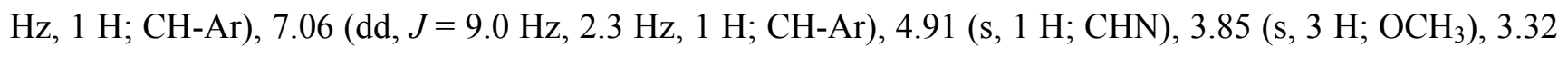
$\left(\mathrm{d}, J=17.0 \mathrm{~Hz}, 1 \mathrm{H} ; \mathrm{CH}_{2} \mathrm{CO}\right), 3.13\left(\mathrm{~m}, 1 \mathrm{H} ; \mathrm{CH}_{2} \mathrm{~N}\right), 2.87$ (td, $\left.J=11.6 \mathrm{~Hz}, 2.5 \mathrm{~Hz}, 1 \mathrm{H} ; \mathrm{CH}_{2} \mathrm{~N}\right), 2.30(\mathrm{~d}, J=$ $\left.17.0 \mathrm{~Hz}, 1 \mathrm{H} ; \mathrm{CH}_{2} \mathrm{CO}\right), 1.88-1.50\left(\mathrm{~m}, 5 \mathrm{H} ; \mathrm{CH}_{2}\right.$ and $\left.\mathrm{NH}\right), 1.32\left(\mathrm{~m}, 2 \mathrm{H} ; \mathrm{CH}_{2}\right), 0.81$ (t, J=7.5 Hz, $3 \mathrm{H} ; \mathrm{CH}_{3}$ ).

${ }^{13} \mathrm{C} \mathrm{NMR}\left(\mathrm{CDCl}_{3}, 100 \mathrm{MHz}\right) \delta 190.7,155.0,132.6,132.1,127.6,118.0,110.6,105.3,103.0,70.7,55.6$, $45.4,40.7,39.2,33.0,30.3,21.8,7.2$.

IR v $2931(\mathrm{w}), 2862(\mathrm{w}), 2834(\mathrm{w}), 1672$ (s), 1531 (s), 1447 (m), $1406(\mathrm{w}), 1351(\mathrm{w}), 1316(\mathrm{w}), 1267$ (w), $1223(\mathrm{~s}), 1179(\mathrm{~m}), 1120(\mathrm{w}), 1032(\mathrm{w}), 918(\mathrm{w}), 860(\mathrm{w}), 845(\mathrm{w}), 799(\mathrm{~m}), 740(\mathrm{~s})$.

HRMS(ESI) calcd for $\mathrm{C}_{18} \mathrm{H}_{23} \mathrm{~N}_{2} \mathrm{O}_{2}^{+}(\mathrm{M}+\mathrm{H}) 299.1760$, found 299.1749.

\section{6-(4-methoxy-1H-indol-2-carbonyl)-5-ethyl-1-azabicyclo[4.1.0]heptane-1-benzylcarboxylate (1c)}

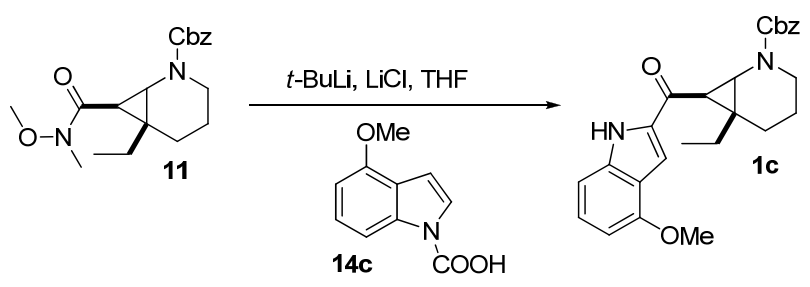

${ }^{t} \mathrm{BuLi}$ (1.6 M in pentane, $0.65 \mathrm{~mL}, 1.0 \mathrm{mmol}, 2.6$ equiv) was added dropwise into a solution of 4-metoxycarboxylindole 14c (100 mg, $0.520 \mathrm{mmol}, 1.30$ equiv) prepared following GP5 and $\mathrm{LiCl}^{[9]}$ (44 mg, 1.0 
mmol, 2.6 equiv) in THF $(3.5 \mathrm{~mL})$ at $-78^{\circ} \mathrm{C}$. The solution was stirred over 3 hours then transferred via cannula into a solution of amide 11 (139 mg, $0.400 \mathrm{mmol}, 1.00$ equiv) in THF $(3.5 \mathrm{~mL})$ at $0^{\circ} \mathrm{C}$ over 25 minutes. The reaction was stirred at $0^{\circ} \mathrm{C}$ over 25 minutes then transferred via cannula into a saturated aqueous solution of $\mathrm{NaHCO}_{3}(10 \mathrm{~mL})$ at $0^{\circ} \mathrm{C}$. The aqueous layer was extracted with $\mathrm{Et}_{2} \mathrm{O}(5 \times 10 \mathrm{~mL})$. The organic layers were dried over $\mathrm{Na}_{2} \mathrm{SO}_{4}$, evaporated under reduced pressure and purified on flash chromatography with deactivated silica (AcOEt/toluene 1:9) to give 1c (86 mg, $20 \mathrm{mmol}, 50 \%)$ as a yellow oil with tendency to solidify.

$R_{f} 0.20$ (AcOEt/PET 3:7, Anisaldehyde).

${ }^{1} \mathrm{H}$ NMR $\left(\mathrm{CDCl}_{3}, 400 \mathrm{MHz}\right) \delta 9.36$ (s, $\left.1 \mathrm{H} ; \mathrm{NH}\right), 7.44-6.96$ (m, $\left.8 \mathrm{H} ; \mathrm{CH}-\mathrm{Ar}\right), 6.52$ (m, $1 \mathrm{H}$; CH-Ar), 5.21-5.07 (m, 0.4 H; $\mathrm{OCH}_{2}$ rotamer B), $5.14\left(J=12.9 \mathrm{~Hz}, 0.8 \mathrm{H} ; \mathrm{OCH}_{2} \operatorname{rotamer} \mathrm{A}\right), 5.02(\mathrm{~d}, J=12.7 \mathrm{~Hz}, 0.8$ $\mathrm{H} ; \mathrm{OCH}_{2}$ rotamer A), $3.96\left(\mathrm{~m}, 4 \mathrm{H} ; \mathrm{OCH}_{3}\right.$ and $\left.\mathrm{NCH}_{2}\right), 3.81(\mathrm{~d}, J=3.1 \mathrm{~Hz}, 1 \mathrm{H} ; \mathrm{CHN}), 2.83(\mathrm{t}, J=12.0 \mathrm{~Hz}$; $0.2 \mathrm{H} ; \mathrm{NCH}_{2}$ rotamer B), 2.74 (t, $\left.J=12.1 \mathrm{~Hz}, 0.8 \mathrm{H} ; \mathrm{NCH}_{2} \operatorname{rotamer} \mathrm{A}\right), 2.63(\mathrm{~d}, J=3.1 \mathrm{~Hz}, 1 \mathrm{H} ; \mathrm{COCH})$, 2.15 (d, $\left.J=14.1 \mathrm{~Hz}, 1 \mathrm{H} ; \mathrm{CH}_{2}\right), 1.89-1.64\left(\mathrm{~m}, 4 \mathrm{H} ; \mathrm{CH}_{2}\right), 1.57$ (m, $\left.1 \mathrm{H} ; \mathrm{CH}_{2}\right), 0.81$ (t, J=7.0 Hz, $3 \mathrm{H} ; \mathrm{CH}_{3}$ ).

${ }^{13} \mathrm{C} \mathrm{NMR}\left(\mathrm{CDCl}_{3}, 100 \mathrm{MHz}\right)$ (rotamers!) $\delta 188.3,156.3,154.8,138.4,136.5,135.6,128.4,128.1,128.0$, $127.5,127.2,127.2,126.9,119.2,106.3,106.0,105.2,105.1,99.6,99.5,67.2,66.9,55.3,45.8,45.5,41.7$, $41.3,38.0,36.5,35.8,26.3,26.1,25.1,21.9,21.8,10.8$.

IR v $3304(\mathrm{w}), 2959(\mathrm{w}), 2936(\mathrm{w}), 2875(\mathrm{w}), 1703$ (s), 1620 (m), 1580 (m), 1517 (s), 1457 (m), 1410 (s), 1364 (s), 1305 (w), 1259 (s), 1239 (w), 1208 (m), 1191 (m), 1162 (s), 1126 (m), 1108 (m), 1079 (w), $1030(\mathrm{w}), 1010(\mathrm{w}), 981(\mathrm{w}), 911(\mathrm{~m}), 824(\mathrm{w}), 776(\mathrm{~m}), 735(\mathrm{~s}), 699(\mathrm{w})$.

HRMS(ESI) calcd for $\mathrm{C}_{26} \mathrm{H}_{29} \mathrm{~N}_{2} \mathrm{O}_{4}{ }^{+}(\mathrm{M}+\mathrm{H})$ 433.2127, found 433.2110.

\author{
Benzyl ethyl-11-methoxy-6-oxo-2,3,4,4a,5,6,7,11c-octahydro-1H-pyrido[3,2-c]carbazole-1-carboxylate \\ (15c); ethyl-11-methoxy-3,4,4a,5,7,11c-hexahydro-1H-pyrido[3,2-c]carbazol-6(2H)-one (25c); benzyl \\ 4a-ethyl-8-methoxy-6-oxo-2,3,4,4a,5,6-hexahydroindolo[1,2-a][1,8]naphthyridine-1(12aH) carboxylate
} (16c) and 4a-ethyl-8-methoxy-1,2,3,4,4a,5-hexahydroindolo[1,2-a][1,8]naphthyridin-6(12aH)-one (26c) 

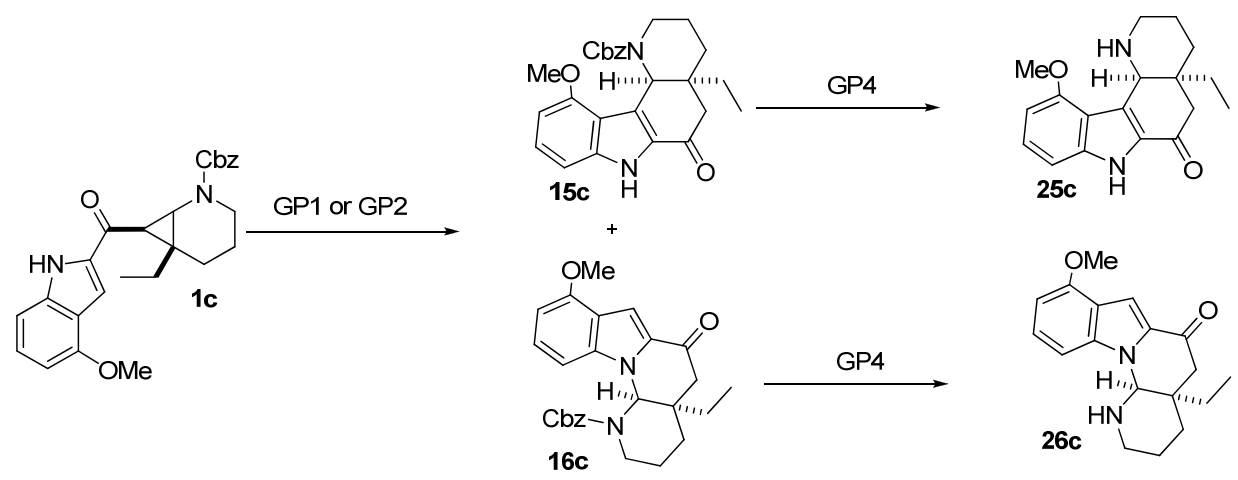

The reaction was performed using general procedure GP2, starting from cyclopropane 1c (40 mg, 92 $\mu \mathrm{mol}, 1.0$ equiv) and copper (II) triflate ( $5.0 \mathrm{mg}, 14 \mu \mathrm{mol}, 0.15$ equiv). The reaction was quenched after 15 min and the crude oil was purified by column chromatography (PET/AcOEt 7:3, Anisaldehyde) to give the product 15c $(R f 0.18,31 \mathrm{mg}, 72 \mu \mathrm{mol}, 78 \%$ ) and the product 16c $(R f 0.20,4 \mathrm{mg}, 9 \mu \mathrm{mol}, 10 \%)$. The tetracyclic compound 15c (30 mg, $70 \mu \mathrm{mol}, 1.0$ equiv) was deprotected following the general procedure GP4 to give 25c (20 mg, $70 \mu \mathrm{mol}$, quant.) as a pale yellow oil without further purification.

The reaction was performed following general procedure GP3, starting from cyclopropane 1c (40 mg, 92 $\mu \mathrm{mol}, 1.0$ equiv) and tosic acid ( $2.6 \mathrm{mg}, 14 \mu \mathrm{mol}, 0.15$ equiv). The reaction was quenched after $15 \mathrm{~min}$ and the crude oil was purified by column chromatography (PET/AcOEt 8:2, Anisaldehyde) to give the product 15c $(R f 0.18,1.7 \mathrm{mg}, 4 \mu \mathrm{mol}, 4 \%)$ and the product 16c $(R f 0.2,35 \mathrm{mg}, 81 \mu \mathrm{mol}, 88 \%)$. The tetracyclic compound 16c (30 mg, $70 \mu \mathrm{mol}, 1.0$ equiv) was deprotected following the general procedure GP4 to give 26c (20 mg, $68 \mu \mathrm{mol}$, quant) as a yellow oil without further purification.

\section{5c}

$R_{f} 0.0$ (AcOEt/PET 6:4 Anisaldehyde).

${ }^{1} \mathrm{H}$ NMR $\left(\mathrm{CDCl}_{3}, 400 \mathrm{MHz}\right) \delta 9.44$ (s br, $1 \mathrm{H} ; \mathrm{NH}$ indole), 7.18 (t, $\left.J=8.0 \mathrm{~Hz}, 1 \mathrm{H} ; \mathrm{CH}-\mathrm{Ar}\right), 6.92(\mathrm{~d}, J=$ $8.2 \mathrm{~Hz}, 1 \mathrm{H}$; CH-Ar), 6.47 (d, J=7.8 Hz, $1 \mathrm{H}$; CH-Ar), 4.24 (s, $1 \mathrm{H}$; CHN), 3.97 (s, $3 \mathrm{H} ; \mathrm{OCH}_{3}$ ), 3.29 (d, $J$ $\left.=16.9 \mathrm{~Hz}, 1 \mathrm{H} ; \mathrm{CH}_{2} \mathrm{CO}\right), 3.19\left(\mathrm{~m}, 1 \mathrm{H} ; \mathrm{CH}_{2} \mathrm{~N}\right), 2.85\left(\mathrm{~d}, J=11.3 \mathrm{~Hz}, 1 \mathrm{H} ; \mathrm{CH}_{2} \mathrm{~N}\right), 2.24(\mathrm{~d}, J=16.9 \mathrm{~Hz}, 1 \mathrm{H}$; $\left.\mathrm{CH}_{2} \mathrm{CO}\right), 1.75\left(\mathrm{~m}, 2 \mathrm{H} ; \mathrm{CH}_{2}\right), 1.62-1.41\left(\mathrm{~m}, 3 \mathrm{H} ; \mathrm{CH}_{2}\right.$ and $\mathrm{NH}$ amine $), 1.30\left(\mathrm{~m}, 2 \mathrm{H} ; \mathrm{CH}_{2}\right), 0.80(\mathrm{t}, J=7.5$ $\left.\mathrm{Hz}, 3 \mathrm{H} ; \mathrm{CH}_{3}\right)$.

${ }^{13} \mathrm{C} \mathrm{NMR}\left(\mathrm{CDCl}_{3}, 100 \mathrm{MHz}\right) \delta 191.1,155.4,139.5,129.5,127.8$ 116.6, 105.7, 99.9, 57.1, 55.5, 45.8, $42.1,40.3,33.4,31.5,21.8,7.7$. (1 $\mathrm{C}$ in aromatic region is not resolved)

IR v 3267 (w), 2960 (w), 2932 (w), 1650 (s), 1620 (w), 1582 (m), 1543 (w), 1518 (w), 1470 (m), 1424 (w), $1391(\mathrm{w}), 1354(\mathrm{~m}), 1266(\mathrm{w}), 1254(\mathrm{~m}), 1166(\mathrm{w}), 1108(\mathrm{~s}), 977$ (w), $919(\mathrm{w}), 873(\mathrm{w}), 785(\mathrm{~m}), 740$ (s), $637(\mathrm{~m}), 617(\mathrm{~s})$. 
HRMS(ESI) calcd for $\mathrm{C}_{18} \mathrm{H}_{23} \mathrm{~N}_{2} \mathrm{O}^{+}(\mathrm{M}+\mathrm{H}) 299.1760$, found 299.1754.

\section{6c}

$R_{f} 0.2$ (AcOEt/PET 4:6 Anisaldehyde).

${ }^{1} \mathrm{H}$ NMR $\left(\mathrm{CDCl}_{3}, 400 \mathrm{MHz}\right) \delta 7.41$ (s, $\left.1 \mathrm{H} ; \mathrm{CH}-\mathrm{Ar}\right), 7.30$ (t, $\left.J=8.1 \mathrm{~Hz}, 1 \mathrm{H} ; \mathrm{CH}-\mathrm{Ar}\right), 6.99$ (d, $J=8.3$ $\mathrm{Hz}, 1 \mathrm{H}$; CH-Ar), 6.51 (d, J=7.7 Hz, $1 \mathrm{H}$; CH-Ar), 4.92 (s, $1 \mathrm{H}$; CHN), 3.94 (s, $3 \mathrm{H}$; OCH $), 3.35$ (d, J= $17.1 \mathrm{~Hz}, 1 \mathrm{H} ; \mathrm{CH}_{2} \mathrm{CO}$ ), 3.13 (m, $\left.1 \mathrm{H} ; \mathrm{CH}_{2} \mathrm{~N}\right), 2.87$ (t, $\left.J=11.8 \mathrm{~Hz}, 1 \mathrm{H} ; \mathrm{CH}_{2} \mathrm{~N}\right), 2.29$ (d, J=17.1 Hz, $1 \mathrm{H}$; $\mathrm{CH}_{2} \mathrm{CO}$ ), 1.87-1.49 (m, $5 \mathrm{H} ; \mathrm{CH}_{2}$ and $\left.\mathrm{NH}\right), 1.33\left(\mathrm{~m}, 2 \mathrm{H} ; \mathrm{CH}_{2}\right), 0.80$ (t, J=7.5 Hz, $3 \mathrm{H} ; \mathrm{CH}_{3}$ ).

${ }^{13} \mathrm{C} \mathrm{NMR}\left(\mathrm{CDCl}_{3}, 100 \mathrm{MHz}\right) \delta 190.5,155.5,138.0,131.2,127.1,118.9,103.8,102.4,100.2,70.7,55.4$, $45.5,40.8,39.2,32.9,30.3,21.7,7.1$.

IR $v 2939$ (w), 2862 (w), 1673 (s), 1615 (w), 1575 (w), 1525 (s), 1505 (w), 1463 (w), 1431 (w), 1368 (w), 1307 (m), 1261 (s), 1182 (m), 1094 (w), 1038 (w), 983 (w), 924 (w), 911 (m), 773 (m), 735 (s).

HRMS(ESI) calcd for $\mathrm{C}_{18} \mathrm{H}_{23} \mathrm{~N}_{2} \mathrm{O}_{2}^{+}(\mathrm{M}+\mathrm{H})$ 299.1760, found 299.1762.

\section{Total synthesis of Goniomitine}

\section{3-Triisopropylyloxyethyl-1H-indole (28)}

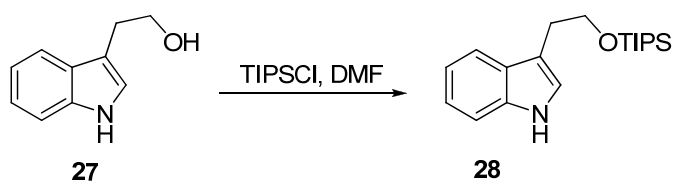

Following a reported procedure, ${ }^{[10]} \operatorname{TIPSCl}(630 \mathrm{mg}, 3.25 \mathrm{mmol}, 1.05$ equiv) was added to a solution of Tryptophol (27) (500 mg, $3.10 \mathrm{mmol}, 1.00$ equiv) and imidazole (440 mg, $6.50 \mathrm{mmol}, 2.10$ equiv) in DMF $(3.5 \mathrm{~mL})$ at RT. The reaction was stirred for 1 hour then quenched with a saturated aqueous solution of $\mathrm{NaHCO}_{3}(5 \mathrm{~mL})$. The organic layer was dried on $\mathrm{Na}_{2} \mathrm{SO}_{4}$ and concentrated under reduced pressure. The crude oil was purified by column chromatography (AcOEt/PET 1:9) to give the product 28 (990 mg, 3.10 mmol, quant.) in quantitative yield.

$R_{f} 0.85$ (AcOEt/PET 2:8 Anisaldehyde).

${ }^{1} \mathrm{H}$ NMR $\left(\mathrm{CDCl}_{3}, 400 \mathrm{MHz}\right) \delta 7.95$ (br s, 1H; NH ), 7.61 (d, J=7.9 Hz, 1H; CH-Ar), 7.35 (d, J=8.1 Hz, $1 \mathrm{H}$; CH-Ar), 7.18 (ddd, $J=8.0 \mathrm{~Hz}, 7.1 \mathrm{~Hz}, 1.1 \mathrm{~Hz}, 1 \mathrm{H}$; CH-Ar), 7.11 (ddd, J=8.0, 7.1, $1.1 \mathrm{~Hz}, 1 \mathrm{H}$; CH- 
Ar), 7.06 (d, $J=1.7 \mathrm{~Hz}, 1 \mathrm{H} ; \mathrm{CH}-\mathrm{Ar}), 3.95$ (t, $\left.J=7.4 \mathrm{~Hz}, 2 \mathrm{H} ; \mathrm{OCH}_{2}\right), 3.03\left(\mathrm{~m}, 2 \mathrm{H} ; \mathrm{CH}_{2}\right), 1.14-1.00$ (m, 21 $\left.\mathrm{H} ;{ }^{i} \mathrm{Pr}\right)$.

The ${ }^{1} \mathrm{H}$ NMR corresponded with literature. ${ }^{[10]}$

\section{6-(3-Triisopropyloxyethyl-1H-indol-2-carbonyl)-5-ethyl-1-azabicyclo[4.1.0]heptane-1-}

\section{benzylcarboxylate (1d)}

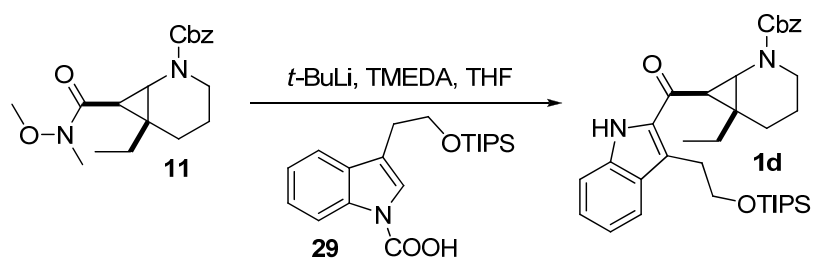

${ }^{t}$ BuLi (1.6 $\mathrm{M}$ in pentane, $0.17 \mathrm{~mL}, 0.28 \mathrm{mmol}, 3.0$ equiv) was added dropwise into a solution of 3triisopropyloxyethyl- $N$-carboxylindole $29(50 \mathrm{mg}, 0.14 \mathrm{mmol}, 1.5$ equiv) prepared following GP5 and TMEDA (21 mg, $0.18 \mathrm{mmol}, 2.0$ equiv) in THF $(1 \mathrm{~mL})$ at $-78^{\circ} \mathrm{C}$. The solution was stirred over 3 hours then transferred via cannula into a solution of amide $11\left(32 \mathrm{mg}, 90 \mu \mathrm{mol}, 1.0\right.$ equiv) in $\mathrm{THF}(1 \mathrm{~mL})$ at $0^{\circ} \mathrm{C}$ over 20 minutes. The reaction was stirred at $0^{\circ} \mathrm{C}$ during 20 minutes then transferred via cannula into a saturated aqueous solution of $\mathrm{NaHCO}_{3}(2 \mathrm{~mL})$ at $0^{\circ} \mathrm{C}$. The aqueous layer was extracted with $\mathrm{Et}_{2} \mathrm{O}(5 \times 5 \mathrm{~mL})$. The organic layers were dried over $\mathrm{Na}_{2} \mathrm{SO}_{4}$, evaporated under reduced pressure and purified on flash chromatography with deactivated silica (AcOEt/PET 1:9) to give amide 11 (8.2 mg, $23 \mu \mathrm{mol}, 25 \%)$ and 1d (20 mg, $33 \mu \mathrm{mol}, 36 \%$ (48\% brsm)) as a yellow oil.

$R_{f} 0.40$ (AcOEt/PET 3:7, Anisaldehyde).

${ }^{1} \mathrm{H}$ NMR $\left(\mathrm{CDCl}_{3}, 400 \mathrm{MHz}\right) \delta 9.84(\mathrm{~s}, 0.2 \mathrm{H}$; NH rotamer B), 8.99 (s, $0.8 \mathrm{H}$; NH rotamer A), 7.70 (d, $J=$ 8.1 Hz, 1 H; CH-Ar), 7.45-7.30 (m, 4 H; CH-Ar), 7.25 (m, 1 H; CH-Ar), 7.20-7.08 (m, 3 H; CH-Ar), 5.28$5.14\left(\mathrm{~m}, 0.4 \mathrm{H} ; \mathrm{OCH}_{2} \operatorname{rotamer} \mathrm{B}\right), 5.18\left(\mathrm{~d}, J=12.7 \mathrm{~Hz}, 0.8 \mathrm{H} ; \mathrm{OCH}_{2} \operatorname{rotamer} \mathrm{A}\right), 5.09$ (d, $J=12.8 \mathrm{~Hz}, 0.8$ $\mathrm{H}$; $\mathrm{OCH}_{2}$ rotamer A), 4.06-3.81 (m, $4 \mathrm{H} ; \mathrm{CH}_{2} \mathrm{O}, \mathrm{NCH}_{2}$ and $\left.\mathrm{NCH}\right), 3.52-3.25\left(\mathrm{~m}, 2 \mathrm{H} ; \mathrm{CH}_{2} \mathrm{C}-\mathrm{Ar}\right), 2.84(\mathrm{~m}$, $0.2 \mathrm{H} ; \mathrm{NCH}_{2}$ rotamer B), 2.83 (d, $J=3.4 \mathrm{~Hz}, 0.8 \mathrm{H} ; \mathrm{CHCO}$ rotamer A), 2.75 (t, $J=11.5 \mathrm{~Hz}, 0.8 \mathrm{H} ; \mathrm{NCH}_{2}$ rotamer A), 2.66 (d, $J=3.2 \mathrm{~Hz}, 0.2 \mathrm{H}$; CHCO rotamer B), $2.29\left(\mathrm{~m}, 1 \mathrm{H} ; \mathrm{CH}_{2}\right), 1.91-1.55\left(\mathrm{~m}, 5 \mathrm{H} ; \mathrm{CH}_{2}\right)$, 1.10-0.97 (m, $\left.21 \mathrm{H} ;{ }^{i} \mathrm{Pr}\right), 0.86$ (m, $\left.3 \mathrm{H} ; \mathrm{CH}_{3}\right)$.

${ }^{13} \mathrm{C} \mathrm{NMR}\left(\mathrm{CDCl}_{3}, 100 \mathrm{MHz}\right)$ (rotamers!) $\delta 189.2,156.2,156.1,136.6,136.5,135.9,135.8,133.6,133.4$, $128.7,128.5,128.4,128.4,128.1,128.0,127.4,127.2,126.9,126.0,125.7,120.9,120.8,120.1,119.9$, $118.5,118.3,112.1,112.0,67.3,66.8,65.2,64.1,45.9,44.5,43.6,41.7,41.2,38.2,38.1,37.5,35.6,29.4$, $29.2,26.1,25.9,25.6,25.3,21.7,21.3,17.9,17.8,11.9,10.7,10.5$. 
IR v 2943 (s), 2866 (m), 2852 (m), 1698 (s), 1556 (w), 1460 (m), 1432 (m), 1386 (w), 1340 (w), 1305 (w), 1268 (m), 1193 (w), 1101 (s), 1013 (w), 919 (w), 884 (w), $820(w), 744$ (s), 695 (m), 633 (s).

HRMS(ESI) calcd for $\mathrm{C}_{36} \mathrm{H}_{51} \mathrm{~N}_{2} \mathrm{O}_{4} \mathrm{Si}^{+}(\mathrm{M}+\mathrm{H})$ 603.3618, found 603.3782 .

Benzyl 4a-ethyl-6-oxo-7-(2-(triisopropylsilyloxy)ethyl)-2,3,4,4a,5,6-hexahydroindolo[1,2a][1,8]naphthyridine-1(12aH)-carboxylate (17)
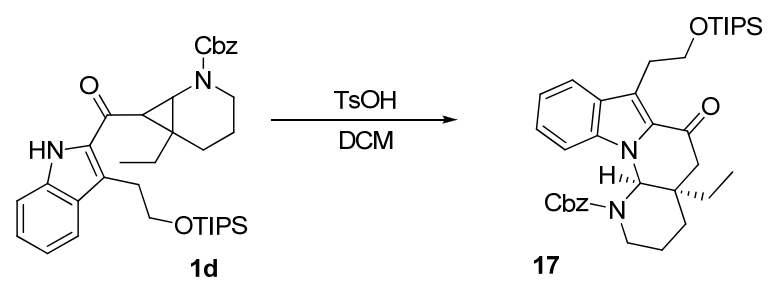

The reaction was performed following general procedure GP3, starting from cyclopropane 1d (60 mg, 0.10 mmol, 1.0 equiv) and tosic acid (3.8 $\mathrm{mg}, 20 \mu \mathrm{mol}, 0.2$ equiv). The reaction was quenched after $10 \mathrm{~min}$, extracted with $\mathrm{Et}_{2} \mathrm{O}(3 \times 10 \mathrm{~mL})$, washed with brine $(2 \times 10 \mathrm{~mL})$ and dried on $\mathrm{Na}_{2} \mathrm{SO}_{4}$. The organic layer was concentrate to give the cyclic product $17(56 \mathrm{mg}, 93 \mu \mathrm{mol}, 93 \%)$ as a yellow oil without further purification.

$R_{f} 0.75$ (AcOEt/PET 2:8 Anisaldehyde).

${ }^{1} \mathrm{H}$ NMR $\left(\mathrm{CDCl}_{3}, 400 \mathrm{MHz}\right) \delta 7.78(\mathrm{~d}, J=8.0 \mathrm{~Hz}, 1 \mathrm{H} ; \mathrm{CH}-\mathrm{Ar}), 7.51-7.34$ (m, $5 \mathrm{H}$; CH-Ar), 7.19-7.09 (m, $2 \mathrm{H}$; CH-Ar), 7.06 (d, J=7.9 Hz, $1 \mathrm{H}$; CH-Ar), 6.43 (br s, $1 \mathrm{H}$; CHN), 5.39 (d, J=11.3 Hz, $1 \mathrm{H}$; $\mathrm{CH}_{2^{-}}$ Ar), $5.31\left(\mathrm{~d}, J=11.6 \mathrm{~Hz}, 1 \mathrm{H} ; \mathrm{CH}_{2}-\mathrm{Ar}\right), 4.06\left(\mathrm{~m}, 1 \mathrm{H} ; \mathrm{CH}_{2} \mathrm{~N}\right), 3.95\left(\mathrm{~m}, 2 \mathrm{H} ; \mathrm{CH}_{2} \mathrm{O}\right), 3.40$ (m, $2 \mathrm{H} ; \mathrm{CH}_{2} \mathrm{C}-$ Ar), 2.67 (d, $J=16.0 \mathrm{~Hz}, 1 \mathrm{H}$; CHCO), 2.49 (d, $J=16.0 \mathrm{~Hz}, 1 \mathrm{H} ; \mathrm{CHCO}), 2.28$ (td, $J=12.9 \mathrm{~Hz}, 2.7 \mathrm{~Hz}, 1 \mathrm{H}$; $\left.\mathrm{CH}_{2} \mathrm{~N}\right), 1.84-1.52\left(\mathrm{~m}, 5 \mathrm{H} ; \mathrm{CH}_{2}\right), 1.30\left(\mathrm{~m}, 1 \mathrm{H} ; \mathrm{CH}_{2}\right), 1.13-0.83\left(\mathrm{~m}, 24 \mathrm{H} ;{ }^{i} \mathrm{Pr}\right.$ and $\left.\mathrm{CH}_{3}\right)$.

${ }^{13} \mathrm{C} \mathrm{NMR}\left(\mathrm{CDCl}_{3}, 100 \mathrm{MHz}\right)$ (rotamers!) $\delta$ 190.0, 155.4, 136.2, 135.6, 129.6, 128.8, 128.6, 128.4, 127.1, $126.7,122.5,122.0,121.1,121.0,120.5,112.2,111.8,68.9,68.0,63.6,47.5,39.0,38.6,28.6,28.2,27.4$, 20.0, 17.9, 17.7, 11.9, 7.5.

IR v 2927 (s), 2863 (m), 1707 (s), 1676 (s), 1545 (m), 1463 (m), 1426 (s), 1346 (m), 1322 (w), 1275 (s), 1217 (m), 1141 (w), $1114(\mathrm{w}), 1101$ (s), 1055 (w), 1020 (w), 964 (w), 885 (w), 742 (s), 694 (m), 633 (m).

HRMS(ESI) calcd for $\mathrm{C}_{18} \mathrm{H}_{23} \mathrm{~N}_{2} \mathrm{O}_{2}^{+}(\mathrm{M}+\mathrm{H})$ 603.3618, found 603.3621 .

2-(4a-Ethyl-1,2,3,4,4a,5,6,12a-octahydro-indolo[1,2-a][1,8]naphthyridin-7-yl)-ethanol (Goniomitine)(3) 

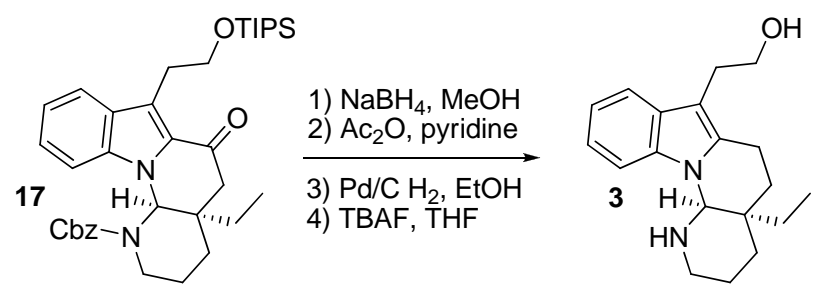

$\mathrm{NaBH}_{4}$ (49.0 mg, $1.30 \mathrm{mmol}, 14.2$ equiv) was added portionwise to a solution of tetracyclic compound 17 (55 mg, $91 \mu \mathrm{mol}, 1.0$ equiv) in $\mathrm{MeOH}(3 \mathrm{~mL})$ at $0^{\circ} \mathrm{C}$. The reaction was allowed to warm to $\mathrm{RT}$ and stirred during 3 hours before adding $5 \mathrm{~mL}$ of ice water and extracting with DCM $(5 \times 5 \mathrm{~mL})$. The organic layer was dried on $\mathrm{Na}_{2} \mathrm{SO}_{4}$ and concentrated under reduced pressure. The white solid obtained was solubilized with pyridine $(1.2 \mathrm{~mL})$ at $\mathrm{rt}$. and $\mathrm{Ac}_{2} \mathrm{O}(0.8 \mathrm{~mL})$ was added dropwise. The reaction was stirred overnight then quenched with $\mathrm{NaHCO}_{3}(5 \mathrm{~mL})$ and extracted with $\mathrm{DCM}(3 \times 5 \mathrm{~mL})$. The organic layer was dried with $\mathrm{Na}_{2} \mathrm{SO}_{4}$ and concentrated to give pale yellow oil which was dissolved in EtOH $(7 \mathrm{~mL})$ and deprotected following the general procedure GP4. The crude oil obtained was diluted in THF ( $5 \mathrm{~mL})$. The solution was stirred at RT and TBAF $(0.4 \mathrm{~mL}, 0.4 \mathrm{mmol}, 4.4$ equiv) was added dropwise. The reaction was stirred $30 \mathrm{~min}$ at RT then quenched with water and extracted with DCM $(3 \times 5 \mathrm{~mL})$. The organic layer was dried on $\mathrm{Na}_{2} \mathrm{SO}_{4}$, concentrated and purified on flash chromatography with deactivated silica (AcOEt/PET 1:1) to give goniomitine 3 ( $21 \mathrm{mg}, 70 \mu \mathrm{mol}, 77 \%$ overall).

$R_{f} 0.2\left(\mathrm{AcOEt} / \mathrm{Hexane} / \mathrm{Et}_{3} \mathrm{~N}\right.$ 29:70:1 Anisaldehyde).

${ }^{1} \mathrm{H}$ NMR $\left(\mathrm{CDCl}_{3}, 400 \mathrm{MHz}\right) \delta 7.52$ (d, $J=7.7 \mathrm{~Hz}, 1 \mathrm{H}$; CH-Ar), 7.30 (d, $J=8.0 \mathrm{~Hz}, 1 \mathrm{H}$; CH-Ar), 7.14 (t, $J=7.2 \mathrm{~Hz}, 1 \mathrm{H}$; CH-Ar), 7.08 (t, $J=7.5 \mathrm{~Hz}, 1 \mathrm{H}$; CH-Ar), 4.79 (s, $1 \mathrm{H}$; NCH), 3.83 (t, $J=6.4 \mathrm{~Hz}, 2 \mathrm{H}$; $\left.\mathrm{OCH}_{2}\right), 3.12-2.75\left(\mathrm{~m}, 6 \mathrm{H} ; \mathrm{CH}_{2}\right), 2.52$ (td, $\left.J=13.1 \mathrm{~Hz}, 6.7 \mathrm{~Hz}, 1 \mathrm{H} ; \mathrm{CH}_{2}\right), 1.89$ (d, $\left.J=13.8 \mathrm{~Hz}, 1 \mathrm{H} ; \mathrm{CH}_{2}\right)$, 1.80-1.41 (m, $7 \mathrm{H} ; \mathrm{CH}_{2} \mathrm{OH}$ and $\left.\mathrm{NH}\right), 1.21\left(\mathrm{~m}, 1 \mathrm{H} ; \mathrm{CH}_{2}\right), 0.88\left(\mathrm{t}, J=7.5 \mathrm{~Hz}, 3 \mathrm{H} ; \mathrm{CH}_{3}\right)$.

${ }^{13} \mathrm{C} \mathrm{NMR}\left(\mathrm{CDCl}_{3}, 125 \mathrm{MHz}\right) \delta 135.3,132.7,129.0,120.5,119.5,118.0,108.2,105.9,71.6,62.6,45.6$, $35.1,34.0,28.6,27.8,21.62,21.58,18.5,7.1$.

IR v $3317(\mathrm{w}), 2934$ (s), 2857 (m), 1735 (w), $1651(\mathrm{w}), 1564$ (w), 1463 (s), $1420(\mathrm{w}), 1360$ (m), 1313 (m), $1271(\mathrm{w}), 1240(\mathrm{w}), 1204(\mathrm{w}), 1118(\mathrm{w}), 1047(\mathrm{~m}), 1017(\mathrm{w}), 910(\mathrm{~m}), 872(\mathrm{w}), 740(\mathrm{~s}), 648(\mathrm{w})$.

HRMS(ESI) calcd for $\mathrm{C}_{19} \mathrm{H}_{27} \mathrm{~N}_{2} \mathrm{O}^{+}(\mathrm{M}+\mathrm{H})$ 299.2123, found 299.2119.

The obtained values for ${ }^{1} \mathrm{H}$ NMR fitted perfectly with the reported ones for natural ${ }^{[11]}$ and synthetic ${ }^{[12]}$ goniomitine. The obtained values for ${ }^{13} \mathrm{C}$ NMR fitted perfectly with the reported values for synthetic

[11] L. Randriambola, J. C. Quirion, C. Kanfan, H. P. Husson, Tetrahedron Lett. 1987, 28, 2123.

[12] a) S. Takano, T. Sato, K. Inomata, K. Ogasawara, J. Chem. Soc. Chem. Commun. 1991, 462; b) C. L. Morales, B. L. Pagenkopf, Org. Lett. 2008, 10, 157. We thank Prof. Pagenkopf for a copy of the original NMR data. 
goniomitine, but small differences were apparent when compared with natural goniomitine, probably due to different conditions of measurement. A comparison of the spectra is provided in section 6 .

\section{Cell Assays ${ }^{[13]}$}

The MCF-7 (breast cancer), HCT116 (colon carcinoma), A549 (lung cancer) and PC-3M (prostate cancer) cell lines originate from the American Type Culture (ATCC) and were obtained as a generous gift from Dr. Markus Wartmann, Novartis AG, Switzerland. Cells were seeded at $1.5 \times 10^{3}$ per well into 96 -well microtiter plates and incubated overnight. Compounds were added in serial dilutions on day 1. Subsequently, the plates were incubated for $72 \mathrm{~h}$ and then fixed with $3.3 \% \mathrm{v} / \mathrm{v}$ glutaraldehyde, washed with water and stained with $0.05 \%$ methylene blue. After washing, the dye was eluted with $3 \% \mathrm{v} / \mathrm{v} \mathrm{HCl}$ and the optical density measured at $665 \mathrm{~nm}$ with a photometer. $\mathrm{IC}_{50}$ values were determined by the GraphPad Prim software (San Diego, USA) using the formula $\left(\mathrm{OD}_{\text {treated }}-\mathrm{OD}_{\text {start }}\right) /\left(\mathrm{OD}_{\text {control }}-\mathrm{OD}_{\text {start }}\right) \times 100$. The $\mathrm{IC}_{50}$ is defined as the drug concentration which leads to $50 \%$ of cells per well as compared to control cultures (100\%) at the end of the incubation period. Experiments were performed three times on different days. The MDCK cells (canine kidney) were obtained as a gift from Dr. Stefanie Krämer, ETH Zurich, Switzerland. The MDR-1-MDCK cells are a stably-transfected cell line expressing human P-glycoprotein.

[13] J. Gertsch, F. Feyen, A. Bützberger, B. Gerber, B. Pfeiffer, K. H. Altmann, Chembiochem 2009, 10, 2513. 
6 Spectra of New Compounds

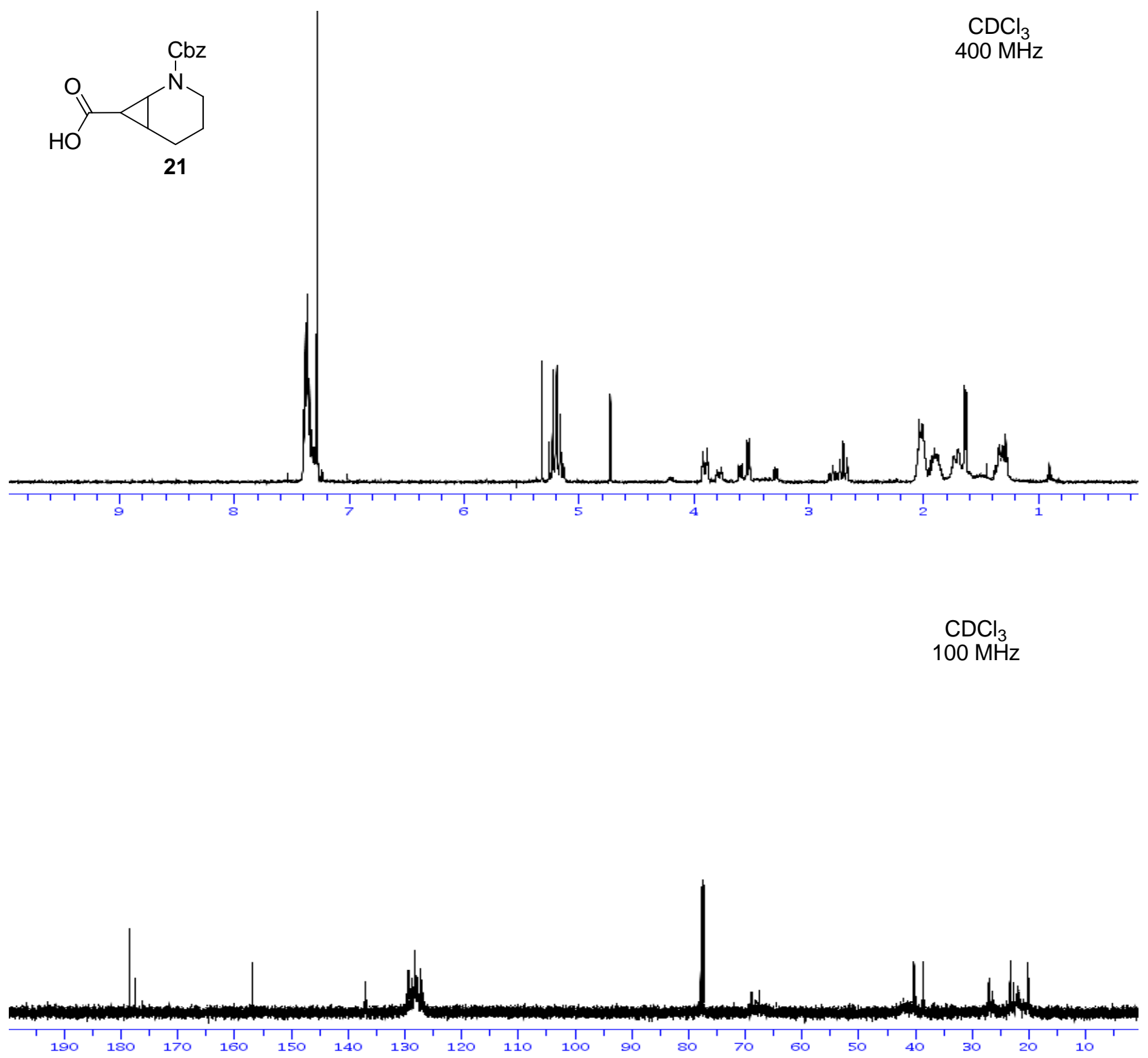



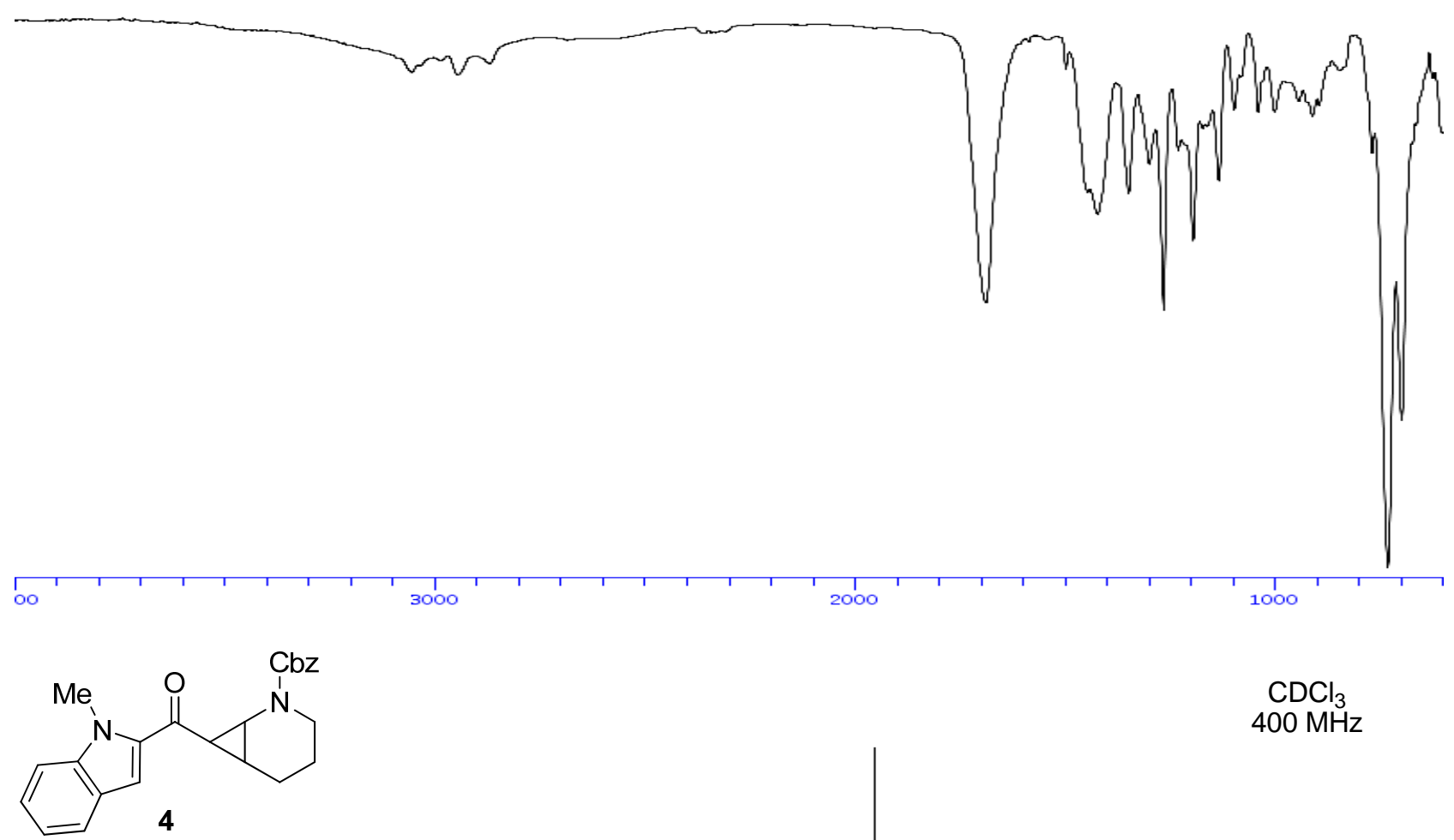

$\mathrm{CDCl}_{3}$

$400 \mathrm{MHz}$

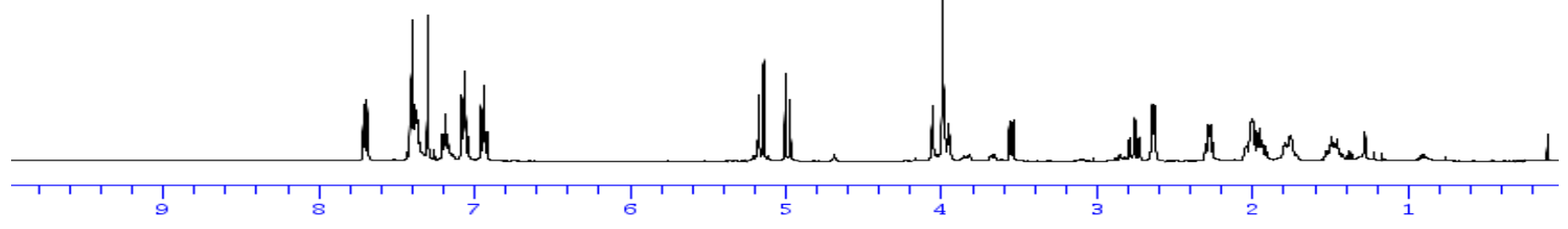

$\mathrm{CDCl}_{3}$

$100 \mathrm{MHz}$

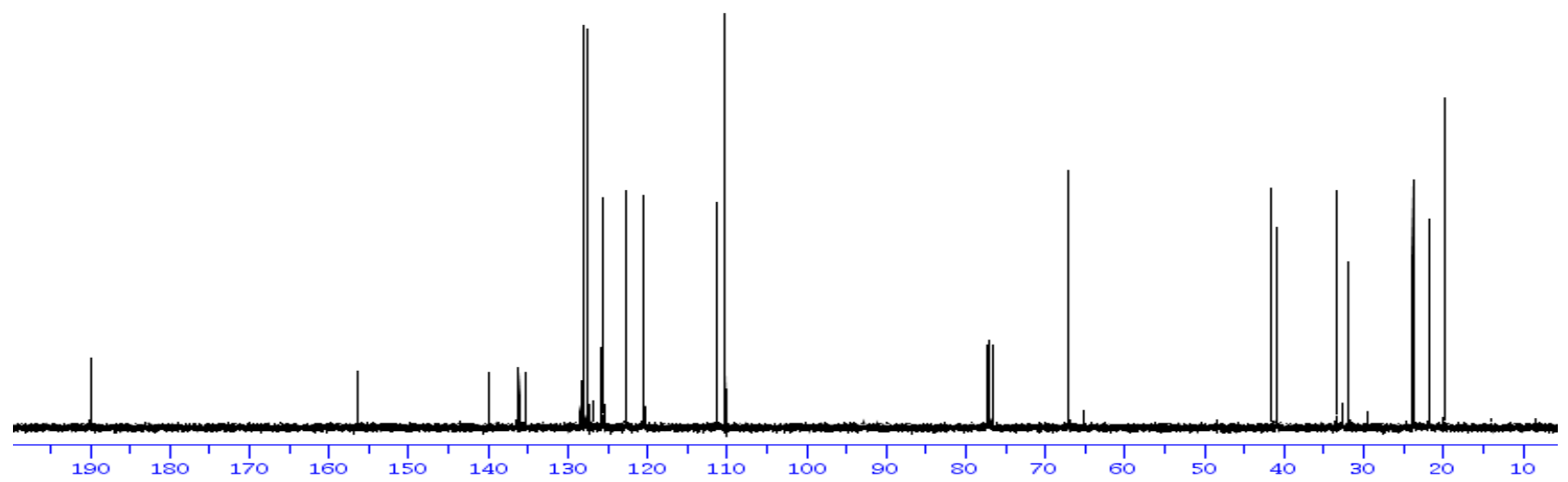



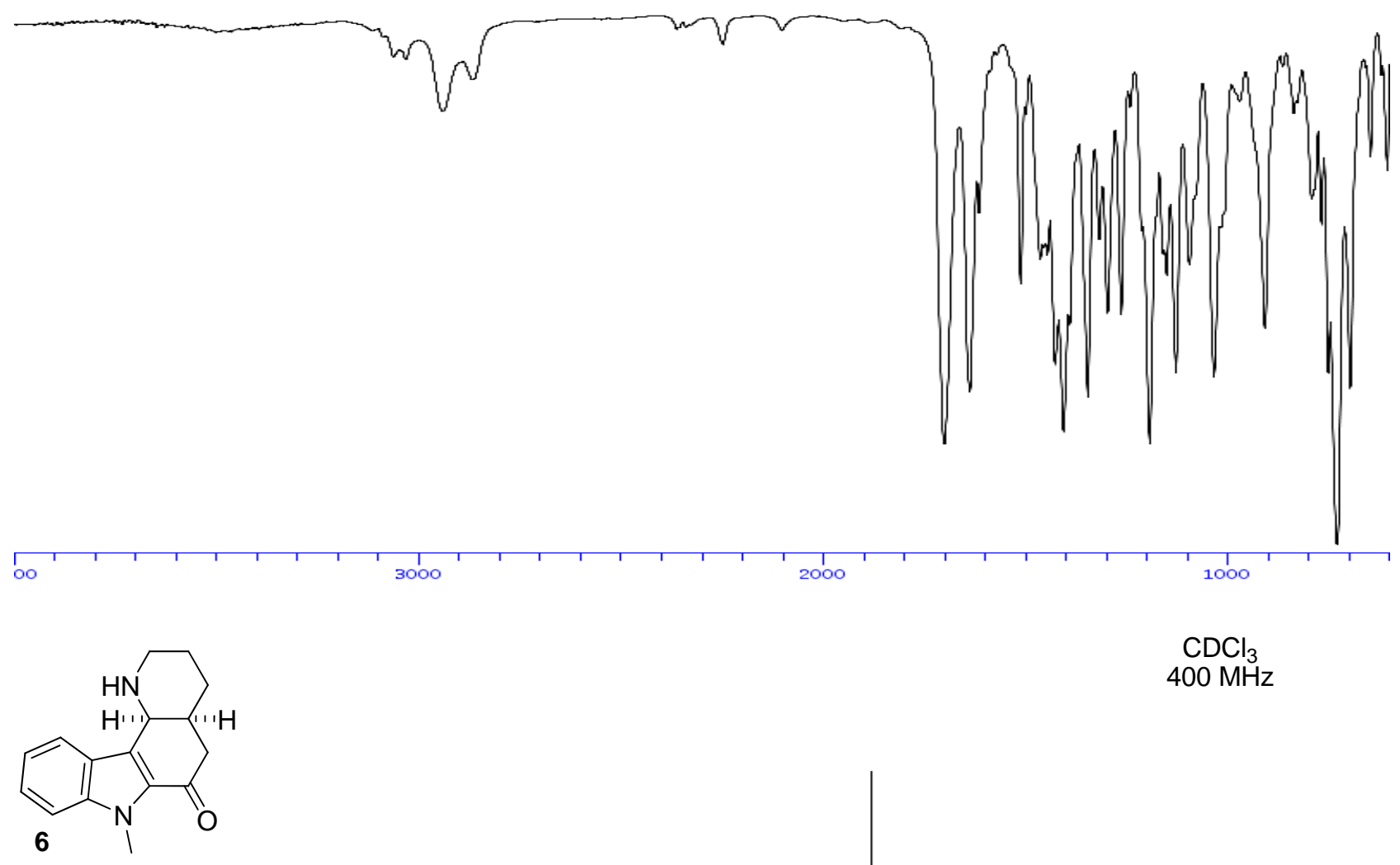

$\mathrm{CDCl}_{3}$ $400 \mathrm{MHz}$

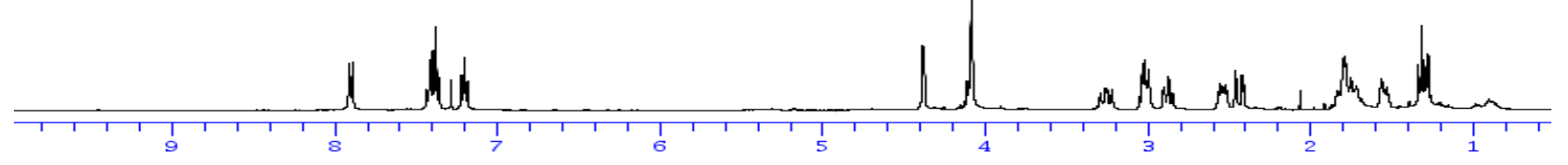

$\mathrm{CDCl}_{3}$

$100 \mathrm{MHz}$

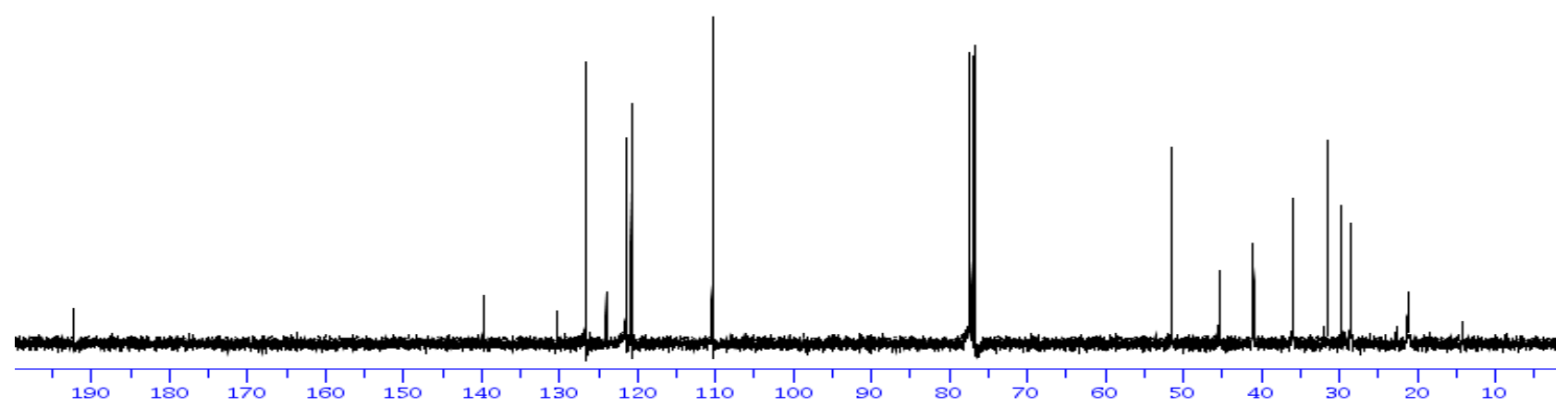



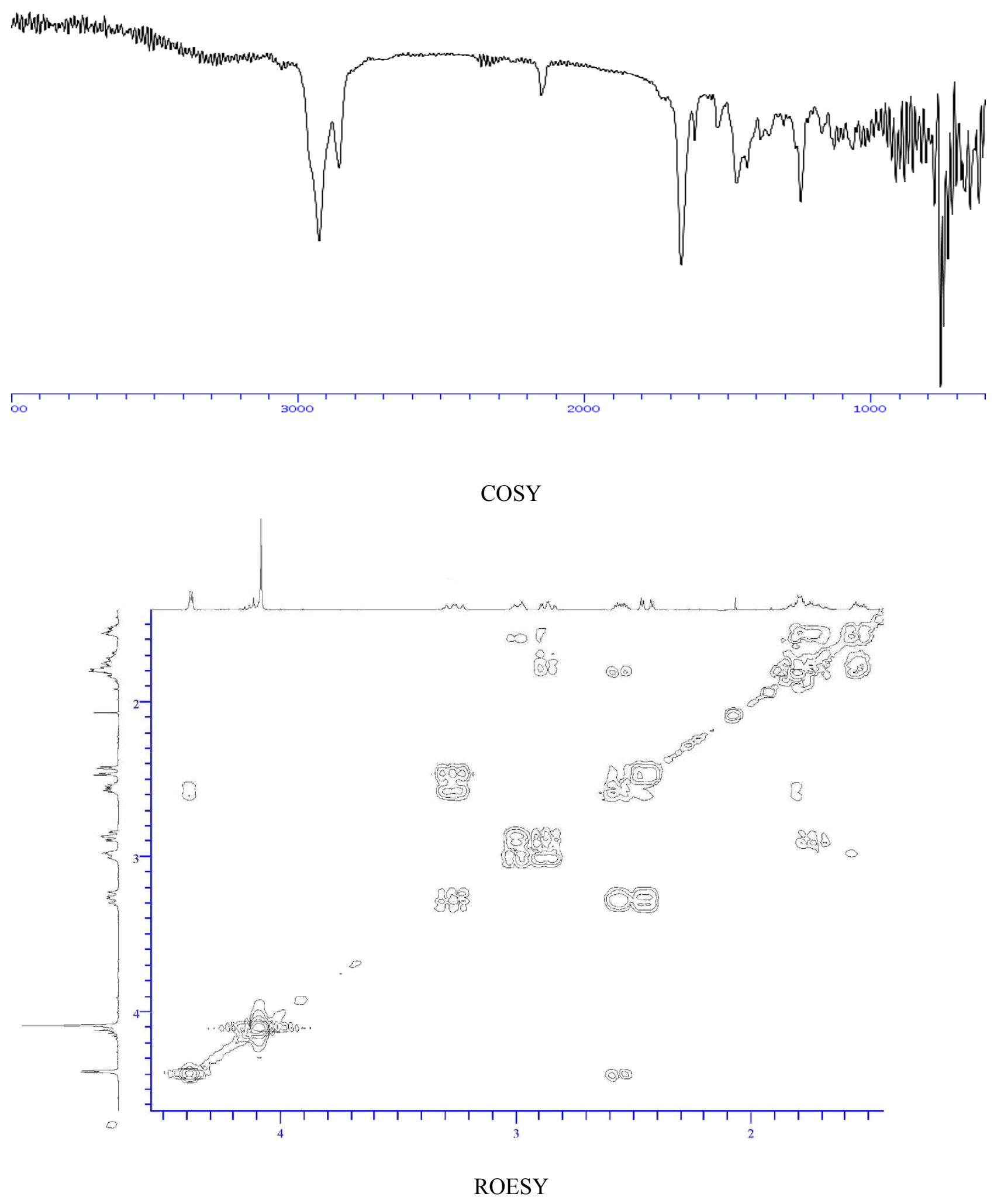


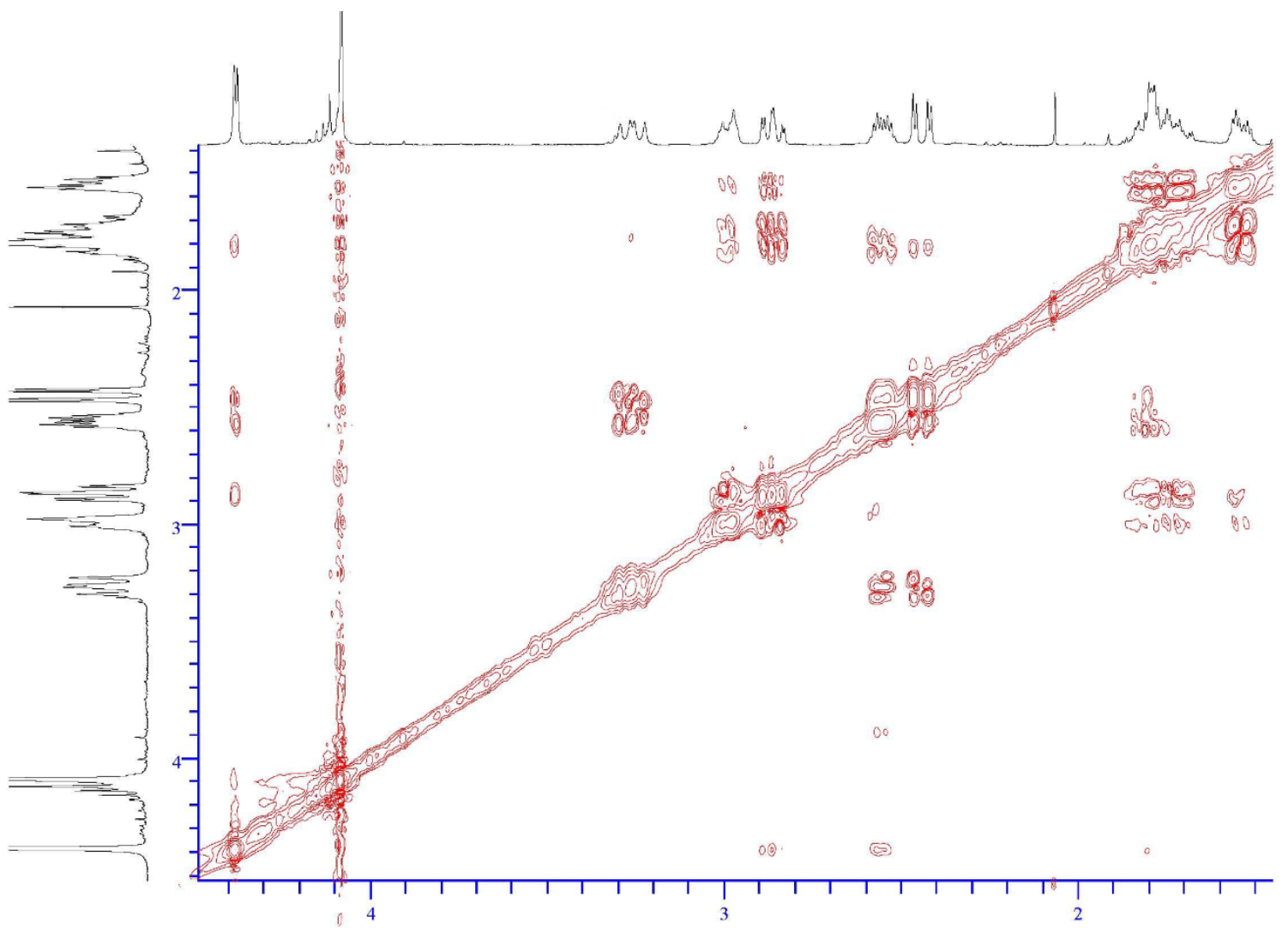


<smiles>CCC1=CN(C(=O)OCc2ccccc2)CC[B]1</smiles>
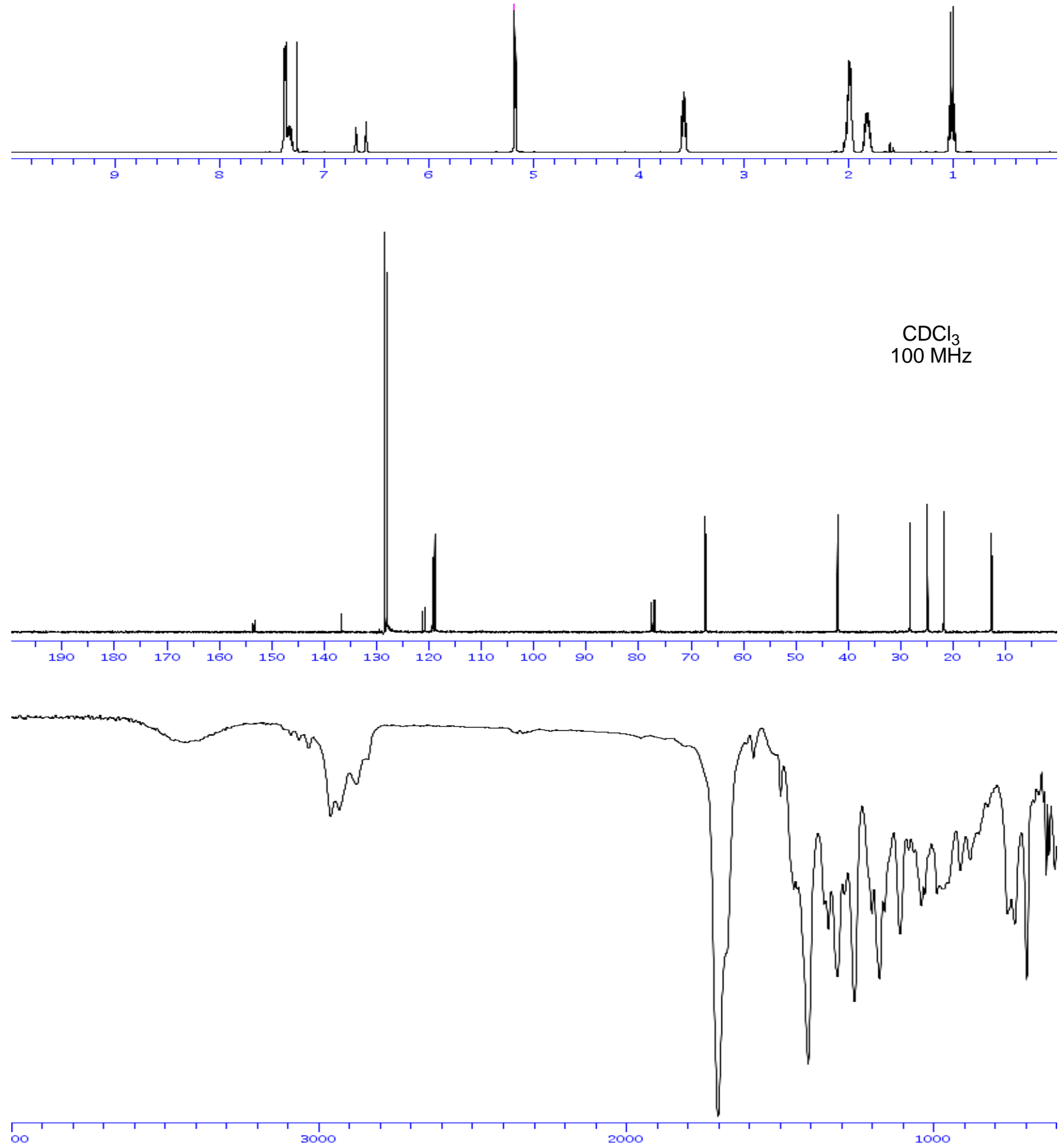
<smiles>CCC12CCCN(C(=O)OC(C)(C)C)C1C2C(=O)O</smiles>
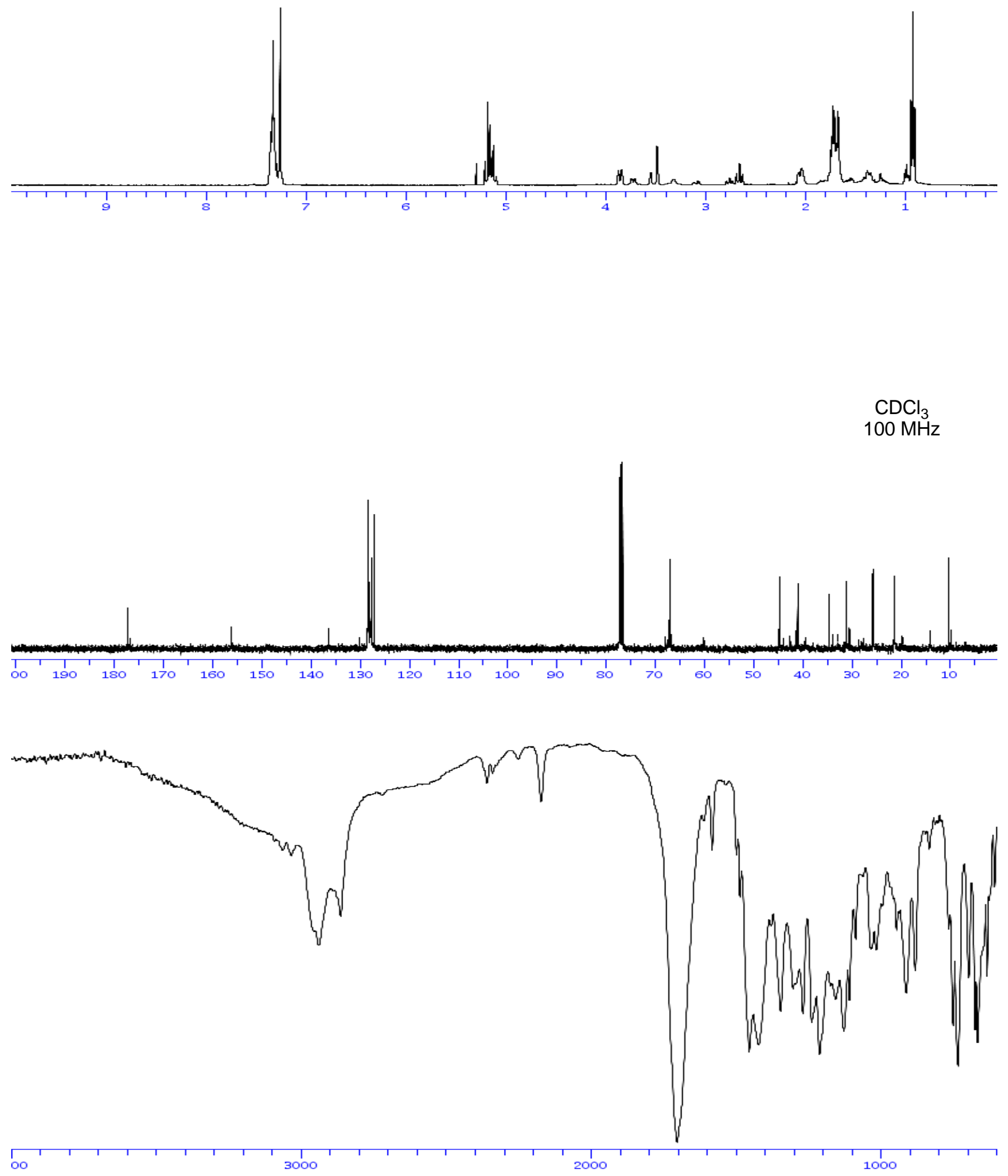

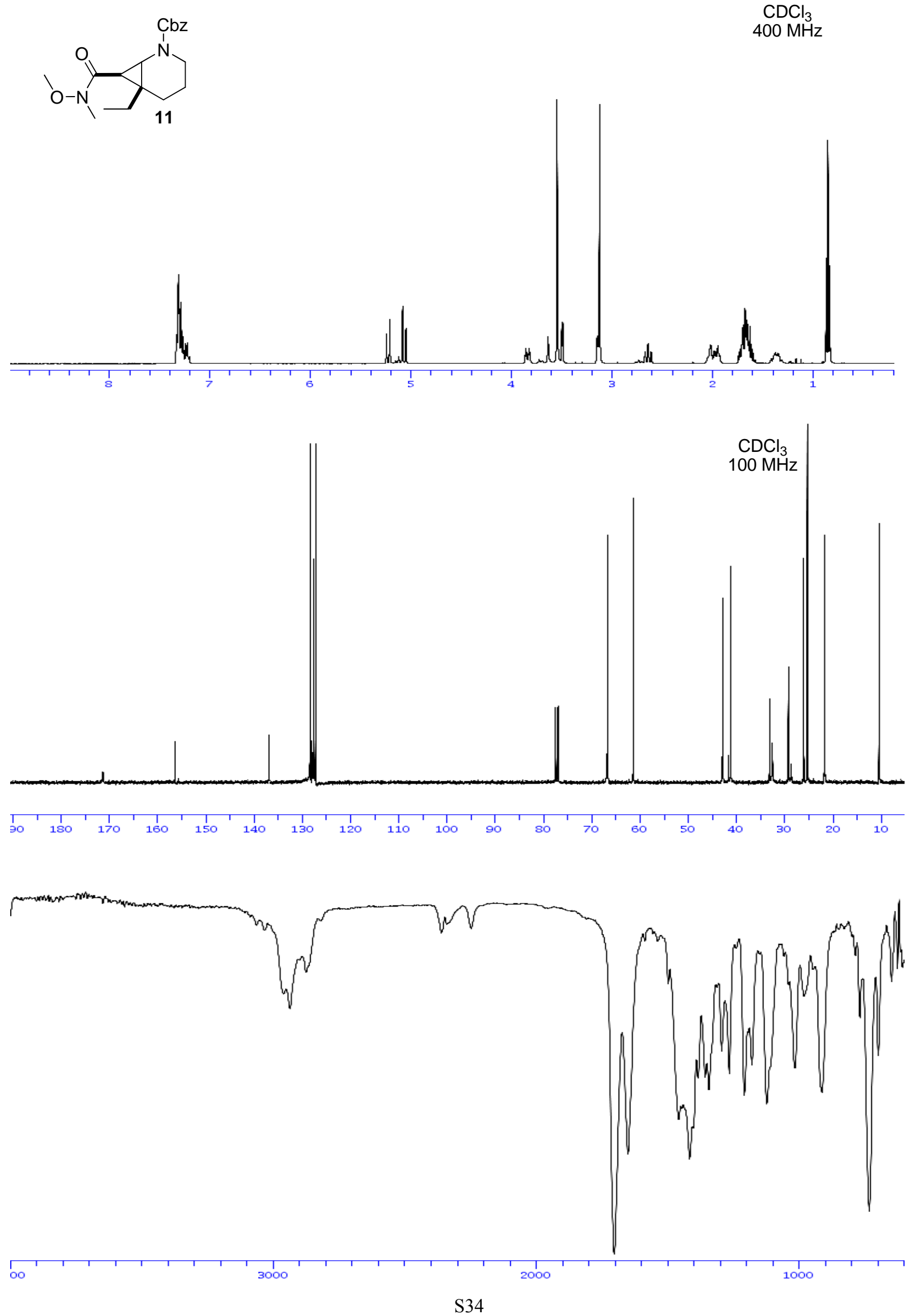

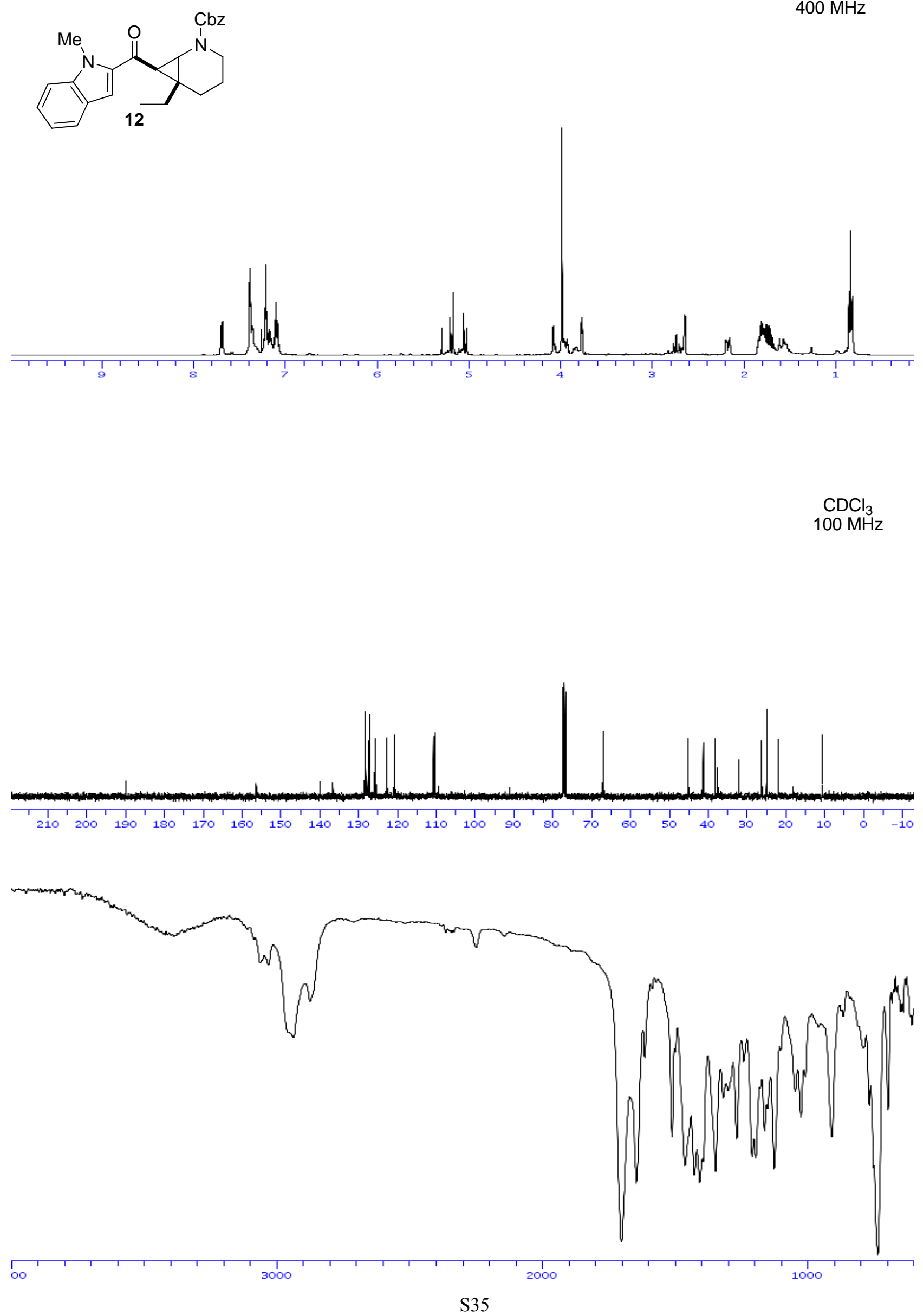


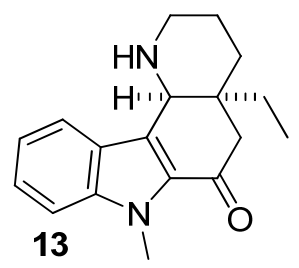

$$
\begin{gathered}
\mathrm{CDCl}_{3} \\
400 \mathrm{MHz}
\end{gathered}
$$
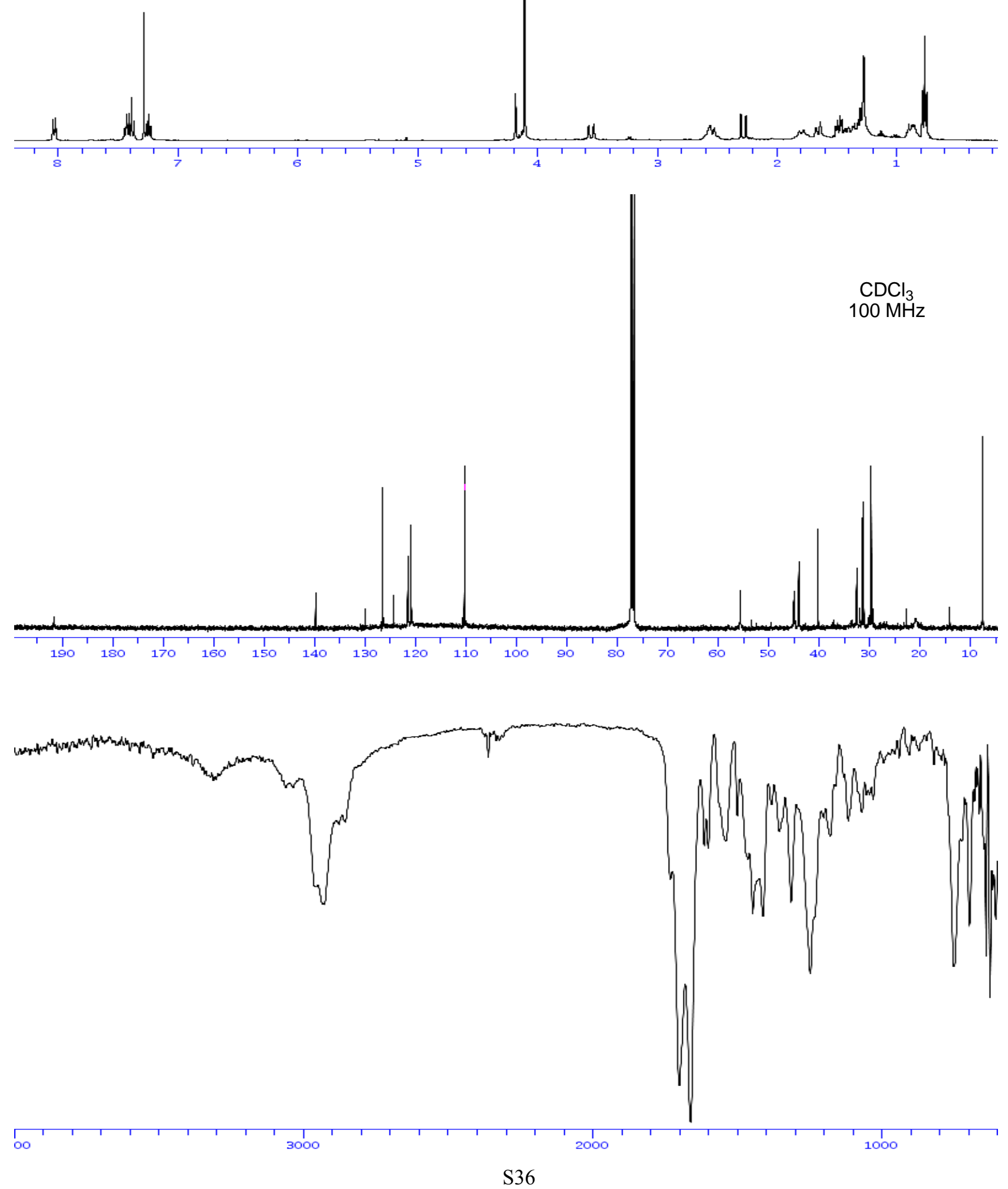
<smiles>CCC12CCCN(C(=O)OC(C)=O)C1C2C(=O)c1cc2ccccc2[nH]1</smiles>
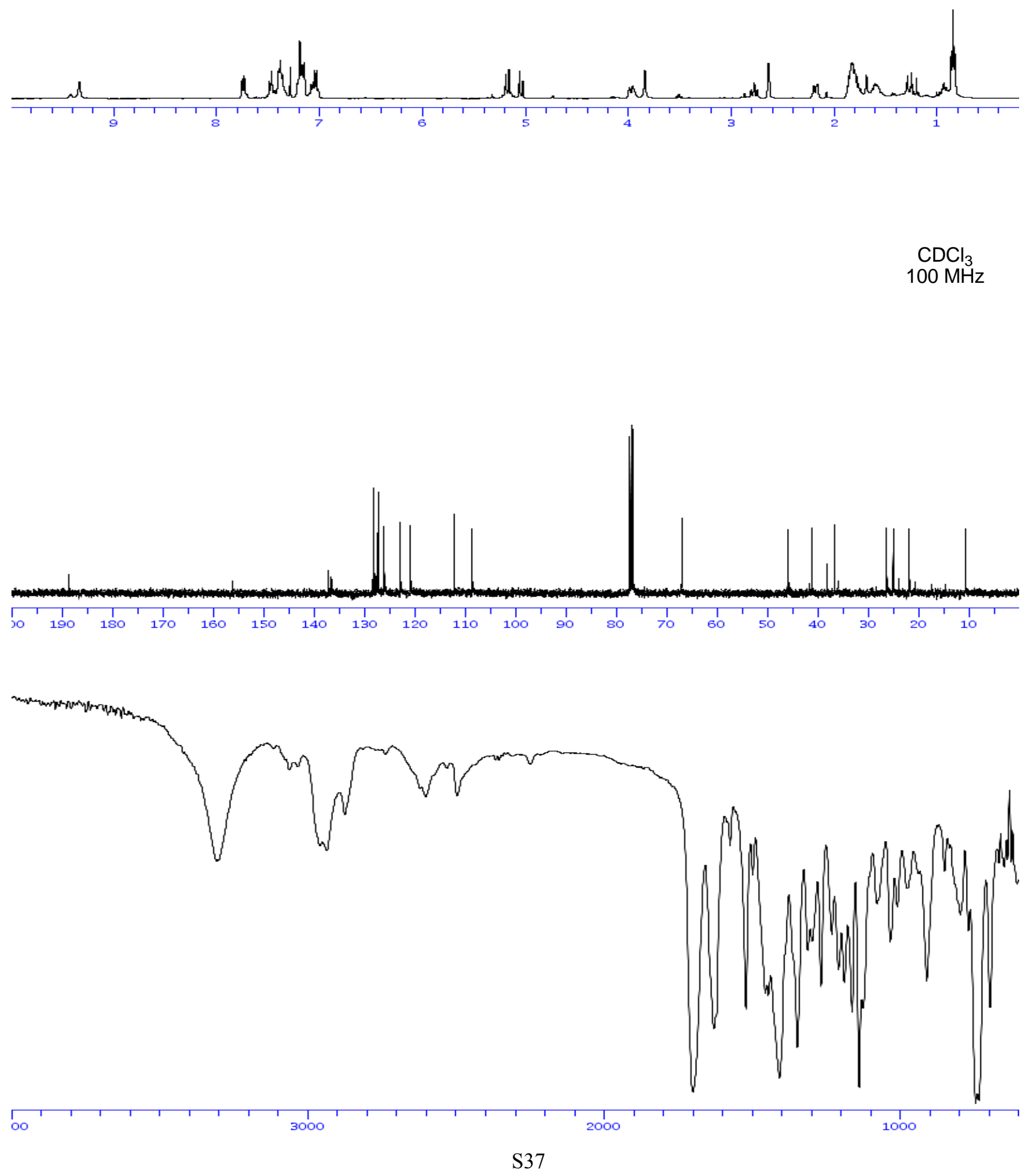


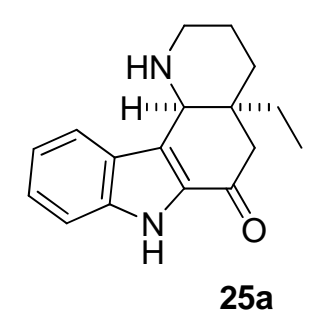

$\mathrm{CD}_{3} \mathrm{OD}$
$400 \mathrm{MHz}$
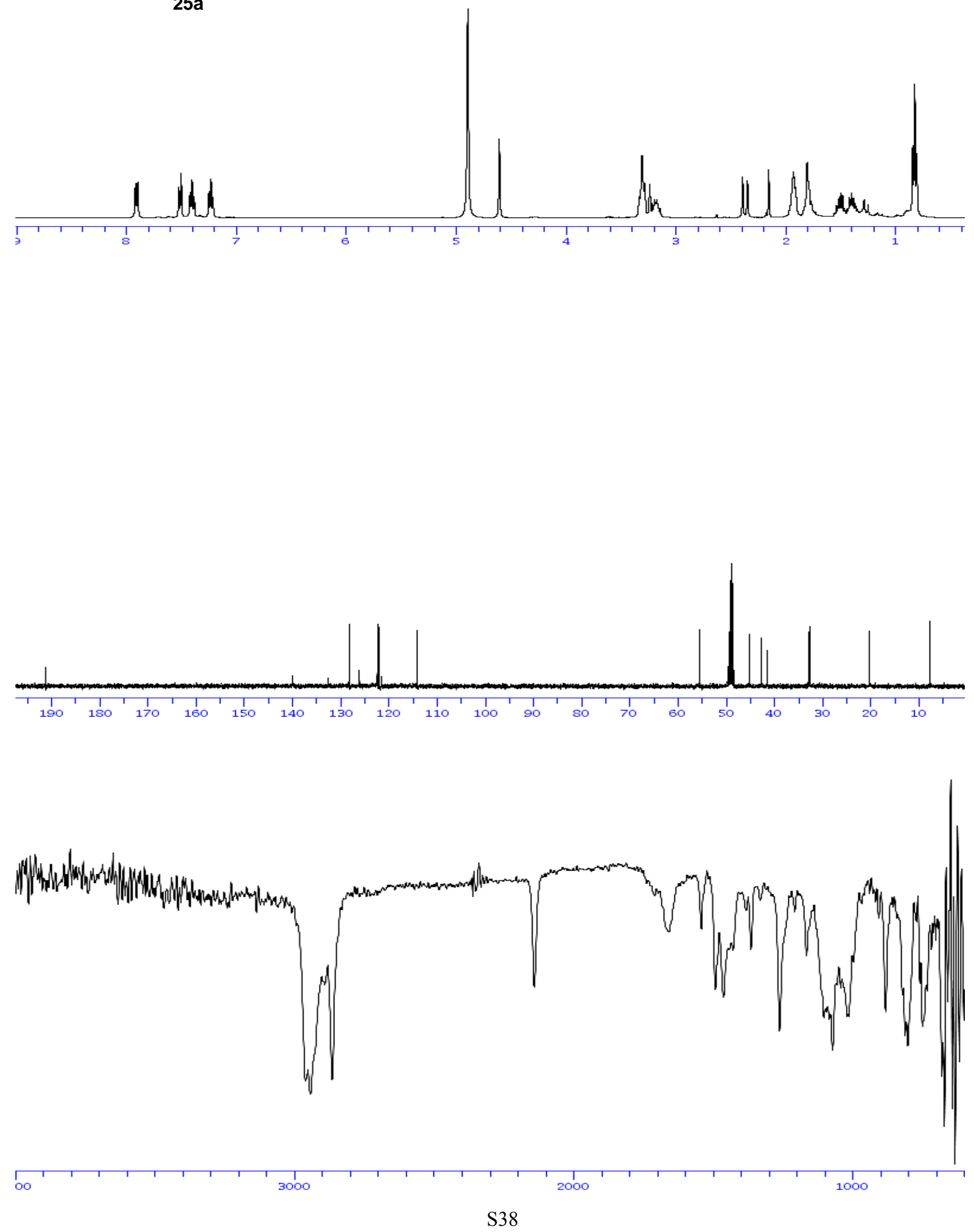


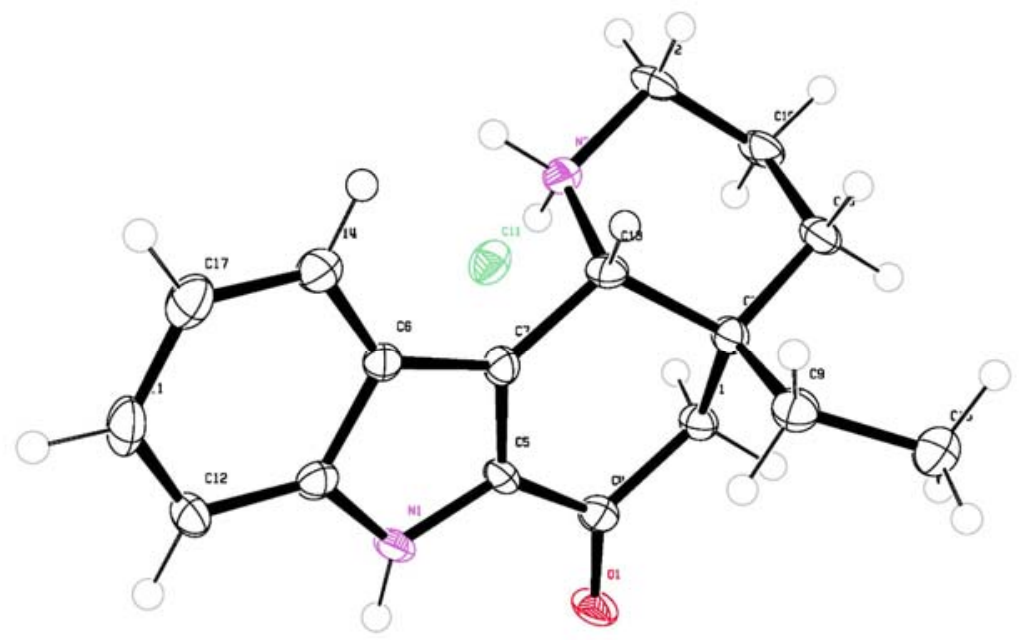

Table 1. Crystal data and structure refinement for fds330.

Identification code

Empirical formula

Formula weight

Temperature

Wavelength

Crystal system

Space group

Unit cell dimensions

Volume

Z

Density (calculated)

Absorption coefficient

$\mathrm{F}(000)$

Crystal size

Theta range for data collection

Index ranges

Reflections collected

Independent reflections

Completeness to theta $=26.37^{\circ}$

Absorption correction

Max. and min. transmission

Refinement method

Data / restraints / parameters fds330

$\mathrm{C} 17 \mathrm{H} 21 \mathrm{ClN} 2 \mathrm{O}$

304.81

140(2) K

$0.71073 \AA$

Monoclinic

$P 2{ }_{1} / c$

$\mathrm{a}=9.1506(10) \AA$

$\alpha=90^{\circ}$.

$\mathrm{b}=15.3882(14) \AA$

$\beta=103.618(12)^{\circ}$.

$\mathrm{c}=10.9477(12) \AA$ $\gamma=90^{\circ}$.

1498.2(3) $\AA^{3}$

4

$1.351 \mathrm{Mg} / \mathrm{m}^{3}$

$0.256 \mathrm{~mm}^{-1}$

648

$0.15 \times 0.11 \times 0.09 \mathrm{~mm}^{3}$

2.93 to $26.37^{\circ}$.

$-11<=\mathrm{h}<=11,-19<=\mathrm{k}<=19,-13<=\mathrm{l}<=13$

13092

$3040[\mathrm{R}(\mathrm{int})=0.0708]$

$99.1 \%$

Semi-empirical from equivalents

1.00000 and 0.92803

Full-matrix least-squares on $\mathrm{F}^{2}$

3040 / 0 / 274 
Goodness-of-fit on $\mathrm{F}^{2}$

Final R indices [I $>2 \operatorname{sigma}(\mathrm{I})]$

$\mathrm{R}$ indices (all data)

Largest diff. peak and hole
0.967

$$
\mathrm{R} 1=0.0546, \mathrm{wR} 2=0.0992
$$$$
\mathrm{R} 1=0.1022, \mathrm{wR} 2=0.1112
$$$$
0.380 \text { and }-0.237 \text { e. } \AA^{-3}
$$

Table 2. Atomic coordinates ( $\left.\times 10^{4}\right)$ and equivalent isotropic displacement parameters $\left(\AA^{2} \times 10^{3}\right)$ for fds330. $U(e q)$ is defined as one third of the trace of the orthogonalized $U^{i j}$ tensor.

\begin{tabular}{|c|c|c|c|c|}
\hline & $\mathrm{x}$ & $\mathrm{y}$ & z & $\mathrm{U}(\mathrm{eq})$ \\
\hline $\mathrm{Cl}(1)$ & $2806(1)$ & $4005(1)$ & $4618(1)$ & $27(1)$ \\
\hline $\mathrm{O}(1)$ & $-709(2)$ & $5221(1)$ & $1532(2)$ & $28(1)$ \\
\hline $\mathrm{N}(1)$ & $3748(3)$ & $5933(2)$ & $4836(2)$ & $19(1)$ \\
\hline $\mathrm{N}(2)$ & 2019(3) & $5647(2)$ & $754(2)$ & $20(1)$ \\
\hline $\mathrm{C}(1)$ & $2842(3)$ & $6581(2)$ & $3930(2)$ & $17(1)$ \\
\hline$C(2)$ & $3758(3)$ & $6081(2)$ & $6187(3)$ & $23(1)$ \\
\hline$C(3)$ & $2167(3)$ & $6082(2)$ & $6324(3)$ & $24(1)$ \\
\hline $\mathrm{C}(4)$ & $1247(3)$ & $6764(2)$ & $5501(3)$ & $21(1)$ \\
\hline $\mathrm{C}(5)$ & $1213(3)$ & $6668(2)$ & $4094(2)$ & $18(1)$ \\
\hline$C(6)$ & $273(3)$ & $5867(2)$ & $3555(2)$ & $18(1)$ \\
\hline$C(7)$ & $317(3)$ & $5612(2)$ & $2231(2)$ & $20(1)$ \\
\hline C(8) & $1700(3)$ & $5838(2)$ & $1889(2)$ & $17(1)$ \\
\hline $\mathrm{C}(9)$ & $2867(3)$ & $6294(2)$ & $2628(2)$ & $17(1)$ \\
\hline $\mathrm{C}(10)$ & 3988(3) & $6389(2)$ & $1933(2)$ & $17(1)$ \\
\hline $\mathrm{C}(11)$ & $3410(3)$ & $5976(2)$ & $759(2)$ & $18(1)$ \\
\hline $\mathrm{C}(12)$ & $4208(3)$ & $5956(2)$ & $-184(3)$ & $23(1)$ \\
\hline $\mathrm{C}(13)$ & $5577(3)$ & $6356(2)$ & $69(3)$ & $27(1)$ \\
\hline$C(14)$ & $6179(3)$ & $6768(2)$ & $1222(3)$ & $26(1)$ \\
\hline $\mathrm{C}(15)$ & $5402(3)$ & $6784(2)$ & 2151(3) & $22(1)$ \\
\hline$C(16)$ & $521(3)$ & $7495(2)$ & $3402(3)$ & $23(1)$ \\
\hline $\mathrm{C}(17)$ & $-898(3)$ & $7839(2)$ & $3712(3)$ & $28(1)$ \\
\hline
\end{tabular}

Table 3. Bond lengths $[\AA]$ and angles $\left[{ }^{\circ}\right]$ for fds330.

\begin{tabular}{ll}
\hline $\mathrm{O}(1)-\mathrm{C}(7)$ & $1.221(3)$ \\
$\mathrm{N}(1)-\mathrm{C}(2)$ & $1.495(3)$ \\
$\mathrm{N}(1)-\mathrm{C}(1)$ & $1.510(3)$ \\
$\mathrm{N}(1)-\mathrm{H}(1 \mathrm{~A})$ & $0.91(3)$ \\
$\mathrm{N}(1)-\mathrm{H}(1 \mathrm{~B})$ & $0.95(3)$ \\
$\mathrm{N}(2)-\mathrm{C}(11)$ & $1.369(3)$
\end{tabular}




\begin{tabular}{|c|c|}
\hline $\mathrm{N}(2)-\mathrm{C}(8)$ & $1.373(3)$ \\
\hline $\mathrm{N}(2)-\mathrm{H}(2)$ & $0.88(3)$ \\
\hline$C(1)-C(9)$ & $1.497(4)$ \\
\hline$C(1)-C(5)$ & $1.548(3)$ \\
\hline $\mathrm{C}(1)-\mathrm{H}(1)$ & $1.00(2)$ \\
\hline$C(2)-C(3)$ & $1.498(4)$ \\
\hline $\mathrm{C}(2)-\mathrm{H}(2 \mathrm{~A})$ & $0.94(3)$ \\
\hline $\mathrm{C}(2)-\mathrm{H}(2 \mathrm{~B})$ & $0.93(3)$ \\
\hline$C(3)-C(4)$ & $1.504(4)$ \\
\hline $\mathrm{C}(3)-\mathrm{H}(3 \mathrm{~A})$ & $0.88(3)$ \\
\hline $\mathrm{C}(3)-\mathrm{H}(3 \mathrm{~B})$ & $1.00(2)$ \\
\hline$C(4)-C(5)$ & $1.541(4)$ \\
\hline $\mathrm{C}(4)-\mathrm{H}(4 \mathrm{~A})$ & $0.98(2)$ \\
\hline $\mathrm{C}(4)-\mathrm{H}(4 \mathrm{~B})$ & $0.96(3)$ \\
\hline$C(5)-C(16)$ & $1.539(4)$ \\
\hline$C(5)-C(6)$ & $1.540(4)$ \\
\hline$C(6)-C(7)$ & $1.511(4)$ \\
\hline $\mathrm{C}(6)-\mathrm{H}(6 \mathrm{~A})$ & $0.97(3)$ \\
\hline $\mathrm{C}(6)-\mathrm{H}(6 \mathrm{~B})$ & $0.97(3)$ \\
\hline $\mathrm{C}(7)-\mathrm{C}(8)$ & $1.444(4)$ \\
\hline$C(8)-C(9)$ & $1.371(3)$ \\
\hline$C(9)-C(10)$ & $1.422(4)$ \\
\hline$C(10)-C(15)$ & $1.398(4)$ \\
\hline$C(10)-C(11)$ & $1.420(4)$ \\
\hline$C(11)-C(12)$ & $1.398(4)$ \\
\hline$C(12)-C(13)$ & $1.364(4)$ \\
\hline $\mathrm{C}(12)-\mathrm{H}(12)$ & $0.94(2)$ \\
\hline$C(13)-C(14)$ & $1.404(4)$ \\
\hline $\mathrm{C}(13)-\mathrm{H}(13)$ & $0.84(3)$ \\
\hline$C(14)-C(15)$ & $1.371(4)$ \\
\hline $\mathrm{C}(14)-\mathrm{H}(14)$ & $0.93(3)$ \\
\hline C(15)-H(15) & $0.99(3)$ \\
\hline$C(16)-C(17)$ & $1.513(4)$ \\
\hline $\mathrm{C}(16)-\mathrm{H}(16 \mathrm{~A})$ & $0.96(3)$ \\
\hline $\mathrm{C}(16)-\mathrm{H}(16 \mathrm{~B})$ & $0.95(3)$ \\
\hline $\mathrm{C}(17)-\mathrm{H}(17 \mathrm{~A})$ & $0.92(3)$ \\
\hline $\mathrm{C}(17)-\mathrm{H}(17 \mathrm{~B})$ & $0.95(3)$ \\
\hline $\mathrm{C}(17)-\mathrm{H}(17 \mathrm{C})$ & $0.96(3)$ \\
\hline $\mathrm{C}(2)-\mathrm{N}(1)-\mathrm{C}(1)$ & $115.0(2)$ \\
\hline $\mathrm{C}(2)-\mathrm{N}(1)-\mathrm{H}(1 \mathrm{~A})$ & $104.3(15)$ \\
\hline
\end{tabular}




\begin{tabular}{|c|c|}
\hline $\mathrm{C}(1)-\mathrm{N}(1)-\mathrm{H}(1 \mathrm{~A})$ & $112.1(16)$ \\
\hline $\mathrm{C}(2)-\mathrm{N}(1)-\mathrm{H}(1 \mathrm{~B})$ & $106.6(19)$ \\
\hline $\mathrm{C}(1)-\mathrm{N}(1)-\mathrm{H}(1 \mathrm{~B})$ & $108.9(19)$ \\
\hline $\mathrm{H}(1 \mathrm{~A})-\mathrm{N}(1)-\mathrm{H}(1 \mathrm{~B})$ & $110(2)$ \\
\hline $\mathrm{C}(11)-\mathrm{N}(2)-\mathrm{C}(8)$ & $108.3(2)$ \\
\hline $\mathrm{C}(11)-\mathrm{N}(2)-\mathrm{H}(2)$ & $123(2)$ \\
\hline $\mathrm{C}(8)-\mathrm{N}(2)-\mathrm{H}(2)$ & $129(2)$ \\
\hline $\mathrm{C}(9)-\mathrm{C}(1)-\mathrm{N}(1)$ & $107.5(2)$ \\
\hline C(9)-C(1)-C(5) & $111.5(2)$ \\
\hline $\mathrm{N}(1)-\mathrm{C}(1)-\mathrm{C}(5)$ & $112.1(2)$ \\
\hline $\mathrm{C}(9)-\mathrm{C}(1)-\mathrm{H}(1)$ & $108.7(12)$ \\
\hline $\mathrm{N}(1)-\mathrm{C}(1)-\mathrm{H}(1)$ & $104.7(12)$ \\
\hline $\mathrm{C}(5)-\mathrm{C}(1)-\mathrm{H}(1)$ & $111.9(12)$ \\
\hline $\mathrm{N}(1)-\mathrm{C}(2)-\mathrm{C}(3)$ & $108.6(2)$ \\
\hline $\mathrm{N}(1)-\mathrm{C}(2)-\mathrm{H}(2 \mathrm{~A})$ & $105.5(15)$ \\
\hline $\mathrm{C}(3)-\mathrm{C}(2)-\mathrm{H}(2 \mathrm{~A})$ & $111.8(15)$ \\
\hline $\mathrm{N}(1)-\mathrm{C}(2)-\mathrm{H}(2 \mathrm{~B})$ & $103.2(15)$ \\
\hline $\mathrm{C}(3)-\mathrm{C}(2)-\mathrm{H}(2 \mathrm{~B})$ & $111.5(16)$ \\
\hline $\mathrm{H}(2 \mathrm{~A})-\mathrm{C}(2)-\mathrm{H}(2 \mathrm{~B})$ & $115(2)$ \\
\hline $\mathrm{C}(2)-\mathrm{C}(3)-\mathrm{C}(4)$ & $111.1(2)$ \\
\hline $\mathrm{C}(2)-\mathrm{C}(3)-\mathrm{H}(3 \mathrm{~A})$ & $105(2)$ \\
\hline $\mathrm{C}(4)-\mathrm{C}(3)-\mathrm{H}(3 \mathrm{~A})$ & $109.8(19)$ \\
\hline $\mathrm{C}(2)-\mathrm{C}(3)-\mathrm{H}(3 \mathrm{~B})$ & $107.9(14)$ \\
\hline $\mathrm{C}(4)-\mathrm{C}(3)-\mathrm{H}(3 \mathrm{~B})$ & $110.2(14)$ \\
\hline $\mathrm{H}(3 \mathrm{~A})-\mathrm{C}(3)-\mathrm{H}(3 \mathrm{~B})$ & $113(2)$ \\
\hline$C(3)-C(4)-C(5)$ & $114.1(2)$ \\
\hline $\mathrm{C}(3)-\mathrm{C}(4)-\mathrm{H}(4 \mathrm{~A})$ & $109.8(13)$ \\
\hline $\mathrm{C}(5)-\mathrm{C}(4)-\mathrm{H}(4 \mathrm{~A})$ & $106.3(13)$ \\
\hline $\mathrm{C}(3)-\mathrm{C}(4)-\mathrm{H}(4 \mathrm{~B})$ & $107.8(16)$ \\
\hline $\mathrm{C}(5)-\mathrm{C}(4)-\mathrm{H}(4 \mathrm{~B})$ & $110.4(16)$ \\
\hline $\mathrm{H}(4 \mathrm{~A})-\mathrm{C}(4)-\mathrm{H}(4 \mathrm{~B})$ & $108(2)$ \\
\hline$C(16)-C(5)-C(6)$ & $110.2(2)$ \\
\hline$C(16)-C(5)-C(4)$ & $108.8(2)$ \\
\hline$C(6)-C(5)-C(4)$ & $109.7(2)$ \\
\hline$C(16)-C(5)-C(1)$ & $108.1(2)$ \\
\hline$C(6)-C(5)-C(1)$ & $110.6(2)$ \\
\hline $\mathrm{C}(4)-\mathrm{C}(5)-\mathrm{C}(1)$ & $109.4(2)$ \\
\hline$C(7)-C(6)-C(5)$ & $115.9(2)$ \\
\hline $\mathrm{C}(7)-\mathrm{C}(6)-\mathrm{H}(6 \mathrm{~A})$ & $103.7(14)$ \\
\hline $\mathrm{C}(5)-\mathrm{C}(6)-\mathrm{H}(6 \mathrm{~A})$ & $109.9(14)$ \\
\hline $\mathrm{C}(7)-\mathrm{C}(6)-\mathrm{H}(6 \mathrm{~B})$ & $109.7(14)$ \\
\hline
\end{tabular}




\begin{tabular}{|c|c|}
\hline $\mathrm{C}(5)-\mathrm{C}(6)-\mathrm{H}(6 \mathrm{~B})$ & $105.7(14)$ \\
\hline $\mathrm{H}(6 \mathrm{~A})-\mathrm{C}(6)-\mathrm{H}(6 \mathrm{~B})$ & $112(2)$ \\
\hline $\mathrm{O}(1)-\mathrm{C}(7)-\mathrm{C}(8)$ & $123.2(2)$ \\
\hline $\mathrm{O}(1)-\mathrm{C}(7)-\mathrm{C}(6)$ & $122.6(2)$ \\
\hline $\mathrm{C}(8)-\mathrm{C}(7)-\mathrm{C}(6)$ & $114.2(2)$ \\
\hline $\mathrm{C}(9)-\mathrm{C}(8)-\mathrm{N}(2)$ & $110.0(2)$ \\
\hline $\mathrm{C}(9)-\mathrm{C}(8)-\mathrm{C}(7)$ & $125.3(2)$ \\
\hline $\mathrm{N}(2)-\mathrm{C}(8)-\mathrm{C}(7)$ & $124.7(2)$ \\
\hline $\mathrm{C}(8)-\mathrm{C}(9)-\mathrm{C}(10)$ & $107.2(2)$ \\
\hline $\mathrm{C}(8)-\mathrm{C}(9)-\mathrm{C}(1)$ & $121.9(2)$ \\
\hline $\mathrm{C}(10)-\mathrm{C}(9)-\mathrm{C}(1)$ & $130.8(2)$ \\
\hline$C(15)-C(10)-C(11)$ & $118.8(2)$ \\
\hline$C(15)-C(10)-C(9)$ & $135.2(3)$ \\
\hline $\mathrm{C}(11)-\mathrm{C}(10)-\mathrm{C}(9)$ & $106.1(2)$ \\
\hline $\mathrm{N}(2)-\mathrm{C}(11)-\mathrm{C}(12)$ & $129.5(3)$ \\
\hline $\mathrm{N}(2)-\mathrm{C}(11)-\mathrm{C}(10)$ & $108.4(2)$ \\
\hline $\mathrm{C}(12)-\mathrm{C}(11)-\mathrm{C}(10)$ & $122.0(2)$ \\
\hline $\mathrm{C}(13)-\mathrm{C}(12)-\mathrm{C}(11)$ & $117.0(3)$ \\
\hline $\mathrm{C}(13)-\mathrm{C}(12)-\mathrm{H}(12)$ & $123.9(15)$ \\
\hline $\mathrm{C}(11)-\mathrm{C}(12)-\mathrm{H}(12)$ & $119.0(15)$ \\
\hline$C(12)-C(13)-C(14)$ & $122.2(3)$ \\
\hline $\mathrm{C}(12)-\mathrm{C}(13)-\mathrm{H}(13)$ & $120.3(18)$ \\
\hline $\mathrm{C}(14)-\mathrm{C}(13)-\mathrm{H}(13)$ & $117.4(18)$ \\
\hline$C(15)-C(14)-C(13)$ & $120.8(3)$ \\
\hline $\mathrm{C}(15)-\mathrm{C}(14)-\mathrm{H}(14)$ & $117.9(17)$ \\
\hline $\mathrm{C}(13)-\mathrm{C}(14)-\mathrm{H}(14)$ & $121.3(17)$ \\
\hline $\mathrm{C}(14)-\mathrm{C}(15)-\mathrm{C}(10)$ & 119.1(3) \\
\hline $\mathrm{C}(14)-\mathrm{C}(15)-\mathrm{H}(15)$ & $121.5(15)$ \\
\hline $\mathrm{C}(10)-\mathrm{C}(15)-\mathrm{H}(15)$ & $119.3(15)$ \\
\hline $\mathrm{C}(17)-\mathrm{C}(16)-\mathrm{C}(5)$ & $117.0(3)$ \\
\hline $\mathrm{C}(17)-\mathrm{C}(16)-\mathrm{H}(16 \mathrm{~A})$ & $106.6(16)$ \\
\hline $\mathrm{C}(5)-\mathrm{C}(16)-\mathrm{H}(16 \mathrm{~A})$ & $110.0(16)$ \\
\hline $\mathrm{C}(17)-\mathrm{C}(16)-\mathrm{H}(16 \mathrm{~B})$ & $109.6(17)$ \\
\hline $\mathrm{C}(5)-\mathrm{C}(16)-\mathrm{H}(16 \mathrm{~B})$ & $109.2(16)$ \\
\hline $\mathrm{H}(16 \mathrm{~A})-\mathrm{C}(16)-\mathrm{H}(16 \mathrm{~B})$ & $104(2)$ \\
\hline $\mathrm{C}(16)-\mathrm{C}(17)-\mathrm{H}(17 \mathrm{~A})$ & $109.4(17)$ \\
\hline $\mathrm{C}(16)-\mathrm{C}(17)-\mathrm{H}(17 \mathrm{~B})$ & 111.3(17) \\
\hline $\mathrm{H}(17 \mathrm{~A})-\mathrm{C}(17)-\mathrm{H}(17 \mathrm{~B})$ & $110(2)$ \\
\hline $\mathrm{C}(16)-\mathrm{C}(17)-\mathrm{H}(17 \mathrm{C})$ & $110.1(17)$ \\
\hline $\mathrm{H}(17 \mathrm{~A})-\mathrm{C}(17)-\mathrm{H}(17 \mathrm{C})$ & $108(2)$ \\
\hline $\mathrm{H}(17 \mathrm{~B})-\mathrm{C}(17)-\mathrm{H}(17 \mathrm{C})$ & $108(2)$ \\
\hline
\end{tabular}


Symmetry transformations used to generate equivalent atoms:

Table 4. Anisotropic displacement parameters $\left(\AA^{2} \times 10^{3}\right)$ for fds 330 . The anisotropic displacement factor exponent takes the form: $-2 \pi^{2}\left[\mathrm{~h}^{2} \mathrm{a}^{* 2} \mathrm{U}^{11}+\ldots+2 \mathrm{~h} \mathrm{k} \mathrm{a}^{*} \mathrm{~b}^{*} \mathrm{U}^{12}\right]$

\begin{tabular}{|c|c|c|c|c|c|c|}
\hline & $\mathrm{U}^{11}$ & $\mathrm{U}^{22}$ & $\mathrm{U}^{33}$ & $U^{23}$ & $\mathrm{U}^{13}$ & $\mathrm{U}^{12}$ \\
\hline $\mathrm{Cl}(1)$ & $20(1)$ & $26(1)$ & $35(1)$ & $-2(1)$ & $7(1)$ & $-2(1)$ \\
\hline $\mathrm{O}(1)$ & 19(1) & $45(1)$ & $18(1)$ & $-11(1)$ & $3(1)$ & $-12(1)$ \\
\hline $\mathrm{N}(1)$ & $17(1)$ & $22(1)$ & $17(1)$ & $-1(1)$ & 2(1) & $-1(1)$ \\
\hline $\mathrm{N}(2)$ & $18(1)$ & $31(1)$ & 12(1) & $-5(1)$ & $3(1)$ & $-4(1)$ \\
\hline$C(1)$ & $15(1)$ & $18(1)$ & $16(2)$ & $-2(1)$ & 2(1) & $-5(1)$ \\
\hline $\mathrm{C}(2)$ & 29(2) & $26(2)$ & $12(2)$ & $-2(1)$ & 1(1) & $-2(1)$ \\
\hline$C(3)$ & $26(2)$ & $34(2)$ & 11(2) & $-2(1)$ & $4(1)$ & $-4(1)$ \\
\hline $\mathrm{C}(4)$ & $20(2)$ & $26(2)$ & $17(2)$ & $-6(1)$ & $6(1)$ & $-6(1)$ \\
\hline$C(5)$ & $16(1)$ & $23(1)$ & $17(2)$ & $-3(1)$ & $5(1)$ & $-2(1)$ \\
\hline$C(6)$ & $16(2)$ & $22(2)$ & $17(2)$ & $-1(1)$ & $5(1)$ & $-2(1)$ \\
\hline $\mathrm{C}(7)$ & 21(2) & 24(1) & $15(2)$ & 1(1) & $4(1)$ & $1(1)$ \\
\hline $\mathrm{C}(8)$ & 19(1) & $23(1)$ & $10(1)$ & $-1(1)$ & $4(1)$ & $-1(1)$ \\
\hline $\mathrm{C}(9)$ & $17(1)$ & 21(1) & $14(1)$ & 1(1) & $6(1)$ & 1(1) \\
\hline $\mathrm{C}(10)$ & $17(1)$ & $20(1)$ & $15(1)$ & $4(1)$ & $3(1)$ & $4(1)$ \\
\hline$C(11)$ & $17(1)$ & $20(1)$ & $17(1)$ & $3(1)$ & $1(1)$ & $4(1)$ \\
\hline $\mathrm{C}(12)$ & $25(2)$ & $30(2)$ & $15(2)$ & 1(1) & $7(1)$ & $5(1)$ \\
\hline $\mathrm{C}(13)$ & $24(2)$ & $34(2)$ & $28(2)$ & $6(1)$ & $18(2)$ & $7(1)$ \\
\hline $\mathrm{C}(14)$ & 19(2) & $31(2)$ & $29(2)$ & $4(1)$ & $9(1)$ & $-1(1)$ \\
\hline $\mathrm{C}(15)$ & $17(2)$ & $26(2)$ & 21(2) & $0(1)$ & $4(1)$ & $-1(1)$ \\
\hline $\mathrm{C}(16)$ & $24(2)$ & $23(2)$ & $22(2)$ & $-1(1)$ & $5(1)$ & $-3(1)$ \\
\hline $\mathrm{C}(17)$ & $23(2)$ & $31(2)$ & $29(2)$ & $-1(2)$ & $7(2)$ & 1(2) \\
\hline
\end{tabular}

Table 5. Hydrogen coordinates ( $\left.\mathrm{x} 10^{4}\right)$ and isotropic displacement parameters $\left(\AA^{2} \mathrm{x} 10^{3}\right)$ for fds 330 .

\begin{tabular}{lcccc}
\hline & $\mathrm{x}$ & $\mathrm{y}$ & $\mathrm{z}$ & $\mathrm{U}(\mathrm{eq})$ \\
& & & \\
$\mathrm{H}(1 \mathrm{~A})$ & $3400(30)$ & $5384(17)$ & $4680(20)$ & $10(6)$ \\
$\mathrm{H}(1 \mathrm{~B})$ & $4760(40)$ & $5960(20)$ & $4780(30)$ & $50(10)$ \\
$\mathrm{H}(2)$ & $1480(30)$ & $5340(20)$ & $120(30)$ & $40(10)$ \\
$\mathrm{H}(1)$ & $3410(20)$ & $7140(14)$ & $4114(19)$ & $0(5)$ \\
& & & $\mathrm{S} 44$ &
\end{tabular}




\begin{tabular}{lrrrr}
$\mathrm{H}(2 \mathrm{~A})$ & $4220(30)$ & $6627(17)$ & $6390(20)$ & $17(7)$ \\
$\mathrm{H}(2 \mathrm{~B})$ & $4290(30)$ & $5608(17)$ & $6590(20)$ & $15(7)$ \\
$\mathrm{H}(3 \mathrm{~A})$ & $2230(30)$ & $6205(18)$ & $7120(30)$ & $33(9)$ \\
$\mathrm{H}(3 \mathrm{~B})$ & $1730(30)$ & $5493(17)$ & $6090(20)$ & $12(6)$ \\
$\mathrm{H}(4 \mathrm{~A})$ & $1660(30)$ & $7341(16)$ & $5750(20)$ & $9(6)$ \\
$\mathrm{H}(4 \mathrm{~B})$ & $250(30)$ & $6741(17)$ & $5630(20)$ & $23(7)$ \\
$\mathrm{H}(6 \mathrm{~A})$ & $660(30)$ & $5355(17)$ & $4050(20)$ & $14(7)$ \\
$\mathrm{H}(6 \mathrm{~B})$ & $-750(30)$ & $6003(16)$ & $3590(20)$ & $16(7)$ \\
$\mathrm{H}(12)$ & $3810(30)$ & $5644(16)$ & $-930(20)$ & $13(7)$ \\
$\mathrm{H}(13)$ & $6110(30)$ & $6345(16)$ & $-460(20)$ & $15(7)$ \\
$\mathrm{H}(14)$ & $7100(30)$ & $7047(18)$ & $1370(30)$ & $28(8)$ \\
$\mathrm{H}(15)$ & $5840(30)$ & $7044(16)$ & $2990(20)$ & $20(7)$ \\
$\mathrm{H}(16 \mathrm{~A})$ & $1240(30)$ & $7961(18)$ & $3560(20)$ & $28(8)$ \\
$\mathrm{H}(16 \mathrm{~B})$ & $350(30)$ & $7405(16)$ & $2520(30)$ & $24(8)$ \\
$\mathrm{H}(17 \mathrm{~A})$ & $-1560(30)$ & $7390(17)$ & $3700(20)$ & $21(8)$ \\
$\mathrm{H}(17 \mathrm{~B})$ & $-1360(30)$ & $8270(19)$ & $3130(30)$ & $28(8)$ \\
$\mathrm{H}(17 \mathrm{C})$ & $-670(30)$ & $8095(18)$ & $4530(30)$ & $26(8)$ \\
& & & & \\
\hline
\end{tabular}

Table 6. Torsion angles $\left[{ }^{\circ}\right]$ for fds 330 .

\begin{tabular}{lc}
\hline $\mathrm{C}(2)-\mathrm{N}(1)-\mathrm{C}(1)-\mathrm{C}(9)$ & $-175.9(2)$ \\
$\mathrm{C}(2)-\mathrm{N}(1)-\mathrm{C}(1)-\mathrm{C}(5)$ & $-52.9(3)$ \\
$\mathrm{C}(1)-\mathrm{N}(1)-\mathrm{C}(2)-\mathrm{C}(3)$ & $57.1(3)$ \\
$\mathrm{N}(1)-\mathrm{C}(2)-\mathrm{C}(3)-\mathrm{C}(4)$ & $-57.6(3)$ \\
$\mathrm{C}(2)-\mathrm{C}(3)-\mathrm{C}(4)-\mathrm{C}(5)$ & $57.6(3)$ \\
$\mathrm{C}(3)-\mathrm{C}(4)-\mathrm{C}(5)-\mathrm{C}(16)$ & $-168.6(2)$ \\
$\mathrm{C}(3)-\mathrm{C}(4)-\mathrm{C}(5)-\mathrm{C}(6)$ & $70.7(3)$ \\
$\mathrm{C}(3)-\mathrm{C}(4)-\mathrm{C}(5)-\mathrm{C}(1)$ & $-50.8(3)$ \\
$\mathrm{C}(9)-\mathrm{C}(1)-\mathrm{C}(5)-\mathrm{C}(16)$ & $-74.1(3)$ \\
$\mathrm{N}(1)-\mathrm{C}(1)-\mathrm{C}(5)-\mathrm{C}(16)$ & $165.3(2)$ \\
$\mathrm{C}(9)-\mathrm{C}(1)-\mathrm{C}(5)-\mathrm{C}(6)$ & $46.6(3)$ \\
$\mathrm{N}(1)-\mathrm{C}(1)-\mathrm{C}(5)-\mathrm{C}(6)$ & $-74.0(3)$ \\
$\mathrm{C}(9)-\mathrm{C}(1)-\mathrm{C}(5)-\mathrm{C}(4)$ & $167.5(2)$ \\
$\mathrm{N}(1)-\mathrm{C}(1)-\mathrm{C}(5)-\mathrm{C}(4)$ & $46.9(3)$ \\
$\mathrm{C}(16)-\mathrm{C}(5)-\mathrm{C}(6)-\mathrm{C}(7)$ & $68.6(3)$ \\
$\mathrm{C}(4)-\mathrm{C}(5)-\mathrm{C}(6)-\mathrm{C}(7)$ & $-171.6(2)$ \\
$\mathrm{C}(1)-\mathrm{C}(5)-\mathrm{C}(6)-\mathrm{C}(7)$ & $-50.9(3)$ \\
$\mathrm{C}(5)-\mathrm{C}(6)-\mathrm{C}(7)-\mathrm{O}(1)$ & $-153.3(3)$ \\
$\mathrm{C}(5)-\mathrm{C}(6)-\mathrm{C}(7)-\mathrm{C}(8)$ & $29.2(3)$ \\
$\mathrm{C}(11)-\mathrm{N}(2)-\mathrm{C}(8)-\mathrm{C}(9)$ & $0.5(3)$ \\
& $\mathrm{S} 45$ \\
&
\end{tabular}




$\begin{array}{lc}\mathrm{C}(11)-\mathrm{N}(2)-\mathrm{C}(8)-\mathrm{C}(7) & 178.7(2) \\ \mathrm{O}(1)-\mathrm{C}(7)-\mathrm{C}(8)-\mathrm{C}(9) & 178.3(3) \\ \mathrm{C}(6)-\mathrm{C}(7)-\mathrm{C}(8)-\mathrm{C}(9) & -4.2(4) \\ \mathrm{O}(1)-\mathrm{C}(7)-\mathrm{C}(8)-\mathrm{N}(2) & 0.3(4) \\ \mathrm{C}(6)-\mathrm{C}(7)-\mathrm{C}(8)-\mathrm{N}(2) & 177.8(2) \\ \mathrm{N}(2)-\mathrm{C}(8)-\mathrm{C}(9)-\mathrm{C}(10) & -0.6(3) \\ \mathrm{C}(7)-\mathrm{C}(8)-\mathrm{C}(9)-\mathrm{C}(10) & -178.9(2) \\ \mathrm{N}(2)-\mathrm{C}(8)-\mathrm{C}(9)-\mathrm{C}(1) & -179.2(2) \\ \mathrm{C}(7)-\mathrm{C}(8)-\mathrm{C}(9)-\mathrm{C}(1) & 2.6(4) \\ \mathrm{N}(1)-\mathrm{C}(1)-\mathrm{C}(9)-\mathrm{C}(8) & 98.6(3) \\ \mathrm{C}(5)-\mathrm{C}(1)-\mathrm{C}(9)-\mathrm{C}(8) & -24.6(3) \\ \mathrm{N}(1)-\mathrm{C}(1)-\mathrm{C}(9)-\mathrm{C}(10) & -79.6(3) \\ \mathrm{C}(5)-\mathrm{C}(1)-\mathrm{C}(9)-\mathrm{C}(10) & 157.2(3) \\ \mathrm{C}(8)-\mathrm{C}(9)-\mathrm{C}(10)-\mathrm{C}(15) & 179.4(3) \\ \mathrm{C}(1)-\mathrm{C}(9)-\mathrm{C}(10)-\mathrm{C}(15) & -2.2(5) \\ \mathrm{C}(8)-\mathrm{C}(9)-\mathrm{C}(10)-\mathrm{C}(11) & 0.5(3) \\ \mathrm{C}(1)-\mathrm{C}(9)-\mathrm{C}(10)-\mathrm{C}(11) & 178.9(2) \\ \mathrm{C}(8)-\mathrm{N}(2)-\mathrm{C}(11)-\mathrm{C}(12) & -179.1(3) \\ \mathrm{C}(8)-\mathrm{N}(2)-\mathrm{C}(11)-\mathrm{C}(10) & -0.1(3) \\ \mathrm{C}(15)-\mathrm{C}(10)-\mathrm{C}(11)-\mathrm{N}(2) & -179.4(2) \\ \mathrm{C}(9)-\mathrm{C}(10)-\mathrm{C}(11)-\mathrm{N}(2) & -0.3(3) \\ \mathrm{C}(15)-\mathrm{C}(10)-\mathrm{C}(11)-\mathrm{C}(12) & -0.3(4) \\ \mathrm{C}(9)-\mathrm{C}(10)-\mathrm{C}(11)-\mathrm{C}(12) & 178.8(2) \\ \mathrm{N}(2)-\mathrm{C}(11)-\mathrm{C}(12)-\mathrm{C}(13) & -178.7(3) \\ \mathrm{C}(10)-\mathrm{C}(11)-\mathrm{C}(12)-\mathrm{C}(13) & -0.2(4) \\ \mathrm{C}(11)-\mathrm{C}(12)-\mathrm{C}(13)-\mathrm{C}(14) & 0.4(4) \\ \mathrm{C}(12)-\mathrm{C}(13)-\mathrm{C}(14)-\mathrm{C}(15) & 0.0(4) \\ \mathrm{C}(13)-\mathrm{C}(14)-\mathrm{C}(15)-\mathrm{C}(10) & -0.5(4) \\ \mathrm{C}(11)-\mathrm{C}(10)-\mathrm{C}(15)-\mathrm{C}(14) & \\ \mathrm{C}(9)-\mathrm{C}(10)-\mathrm{C}(15)-\mathrm{C}(14) & \\ \mathrm{C}(6)-\mathrm{C}(5)-\mathrm{C}(16)-\mathrm{C}(17) & \\ \mathrm{C}(4)-\mathrm{C}(5)-\mathrm{C}(16)-\mathrm{C}(17) & \\ \mathrm{C}(1)-\mathrm{C}(5)-\mathrm{C}(16)-\mathrm{C}(17) & \\ & \\ & \end{array}$

Symmetry transformations used to generate equivalent atoms:

Table 7. Hydrogen bonds for fds330 [ $\AA$ and $\left.{ }^{\circ}\right]$.

\begin{tabular}{lcccc}
\hline D-H...A & d(D-H) & d(H...A & d(D...A & $<($ DHA $)$ \\
\hline N(1)-H(1A)..Cl(1) & $0.91(3)$ & $2.19(3)$ & $3.082(3)$ & $170(2)$ \\
& & & \multicolumn{2}{c}{ S46 }
\end{tabular}


$\mathrm{N}(1)-\mathrm{H}(1 \mathrm{~B}) \ldots \mathrm{Cl}(1) \#$

$0.95(3)$

$2.17(3)$

$3.069(2)$

159(3)

$\mathrm{N}(2)-\mathrm{H}(2) \ldots \mathrm{O}(1) \# 2$

$0.88(3)$

1.98(3)

2.841(3)

164(3)

Symmetry transformations used to generate equivalent atoms:

$\# 1-x+1,-y+1,-z+1 \quad \# 2-x,-y+1,-z$ 

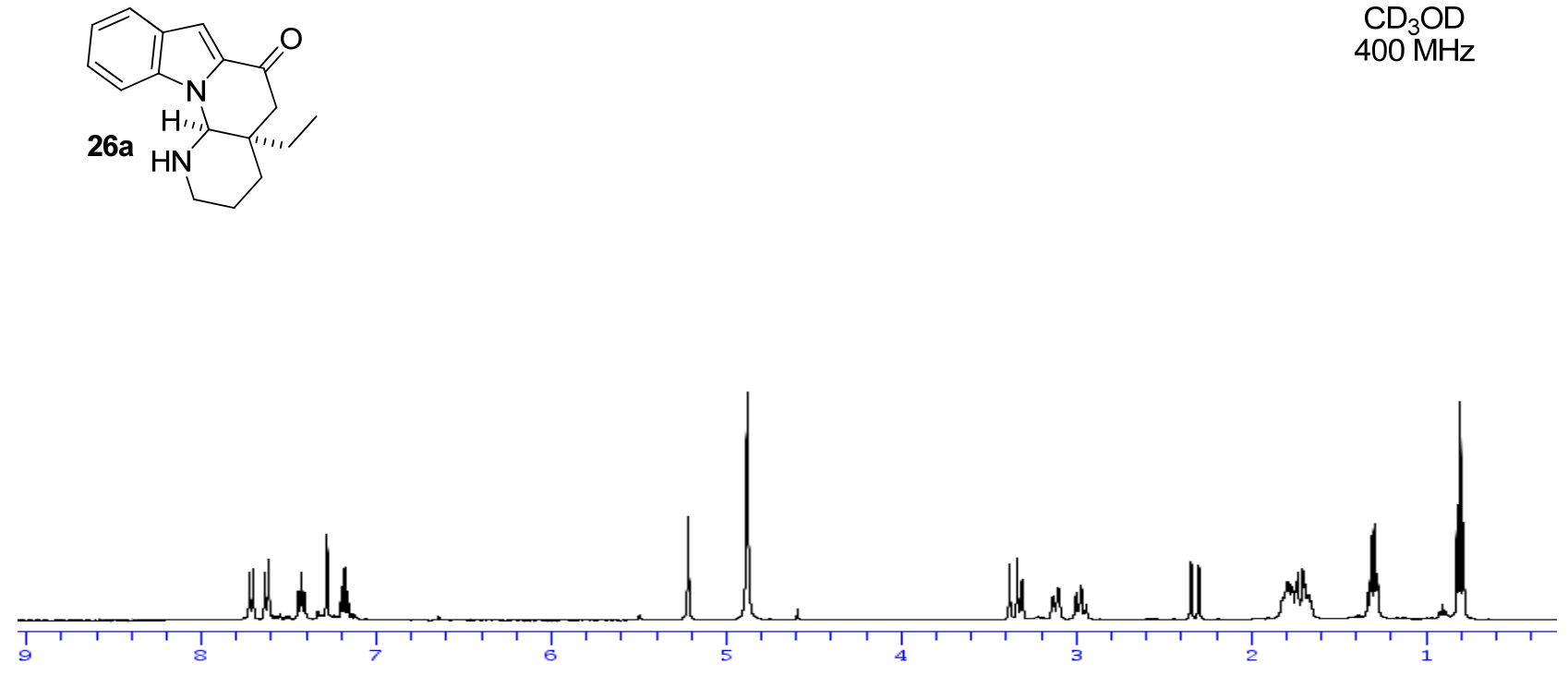

$\mathrm{CD}_{3} \mathrm{OD}$

$125 \mathrm{MHz}$

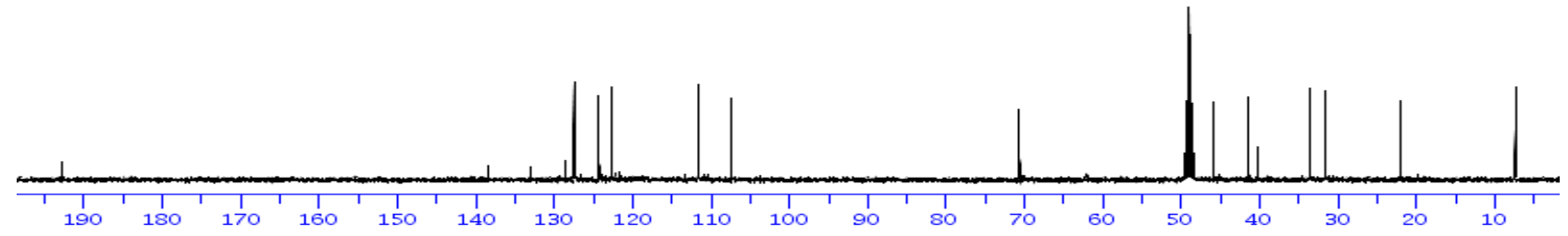




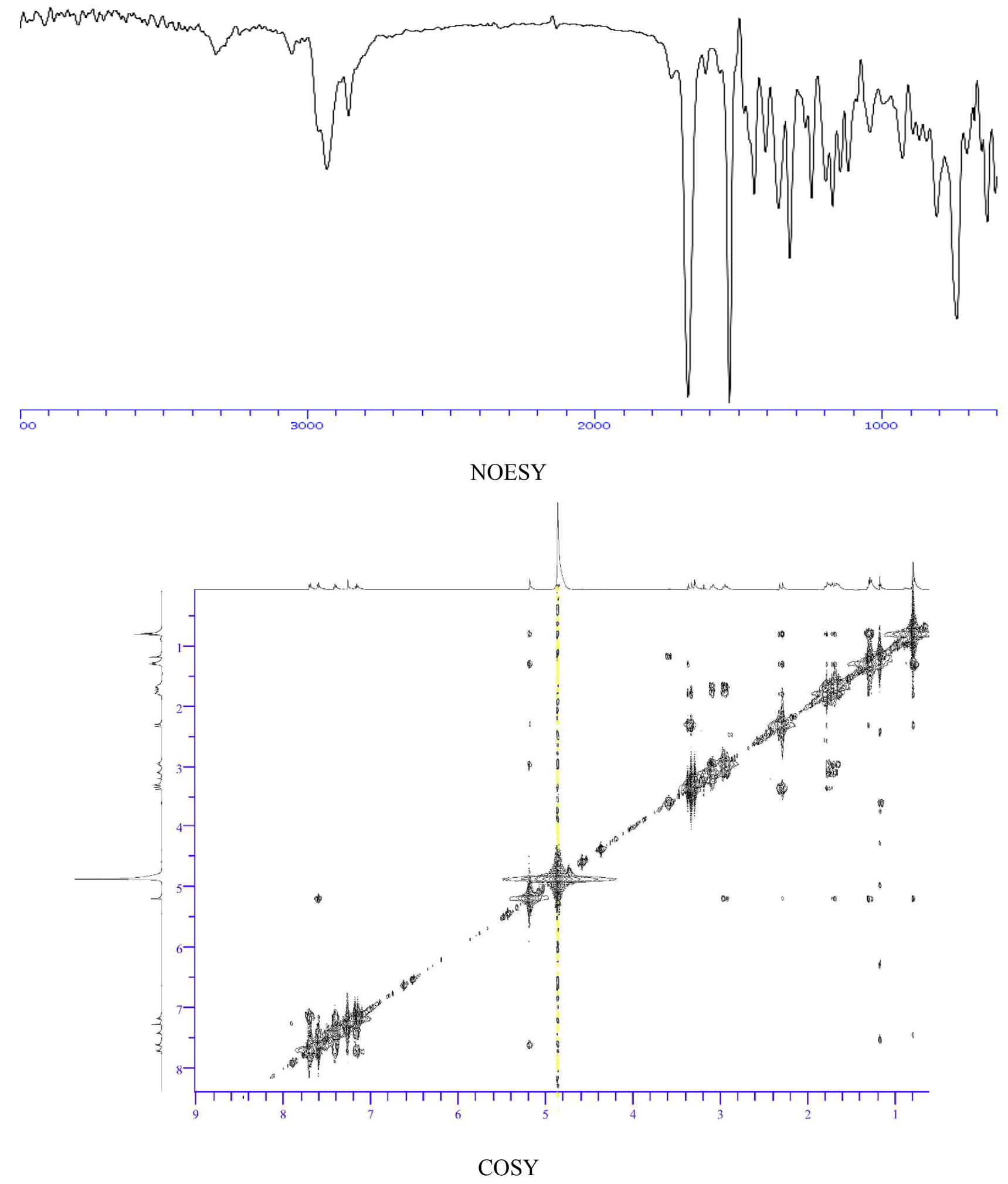



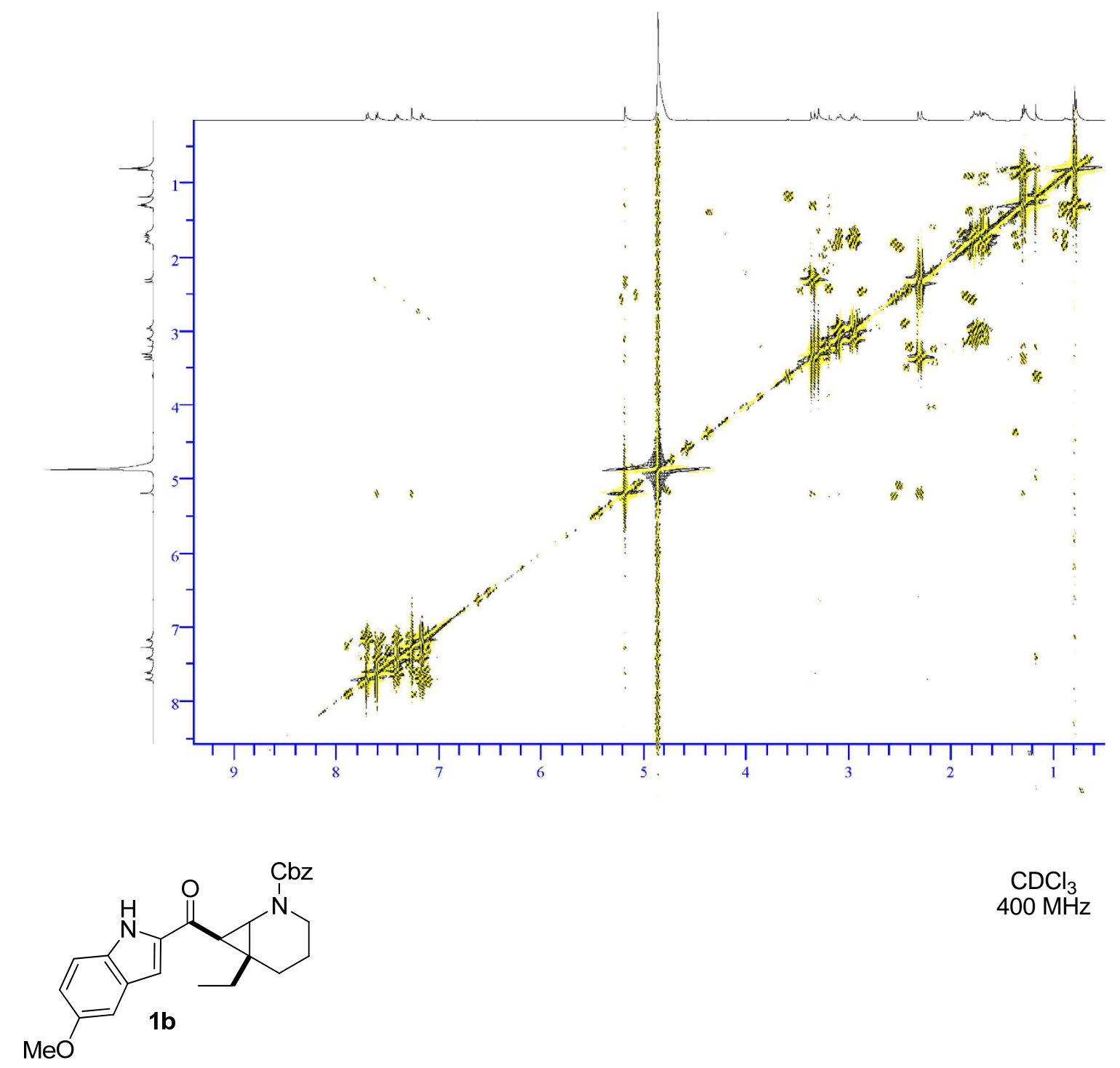

$\mathrm{CDCl}_{3}$

$400 \mathrm{MHz}$

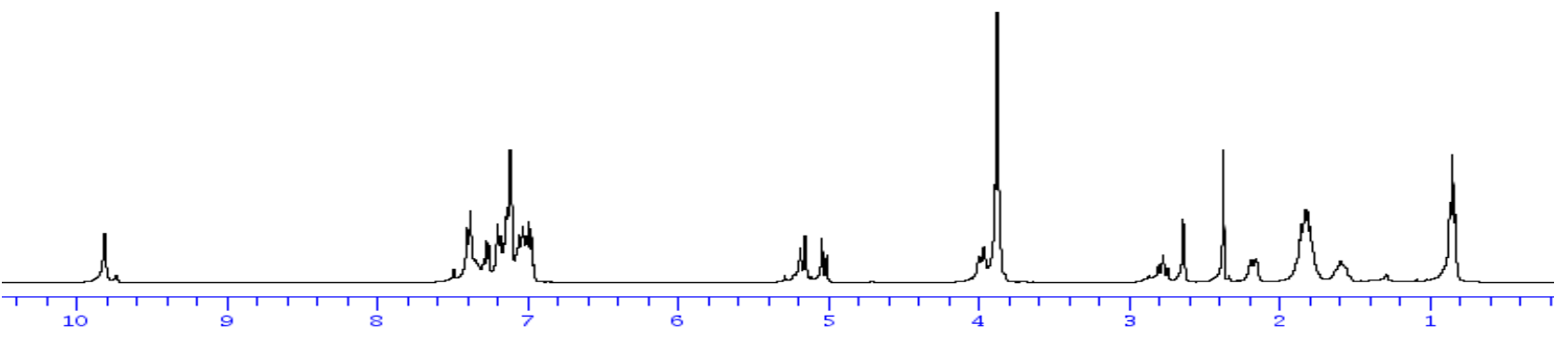


$\mathrm{CDCl}_{3}$

$100 \mathrm{MHz}$
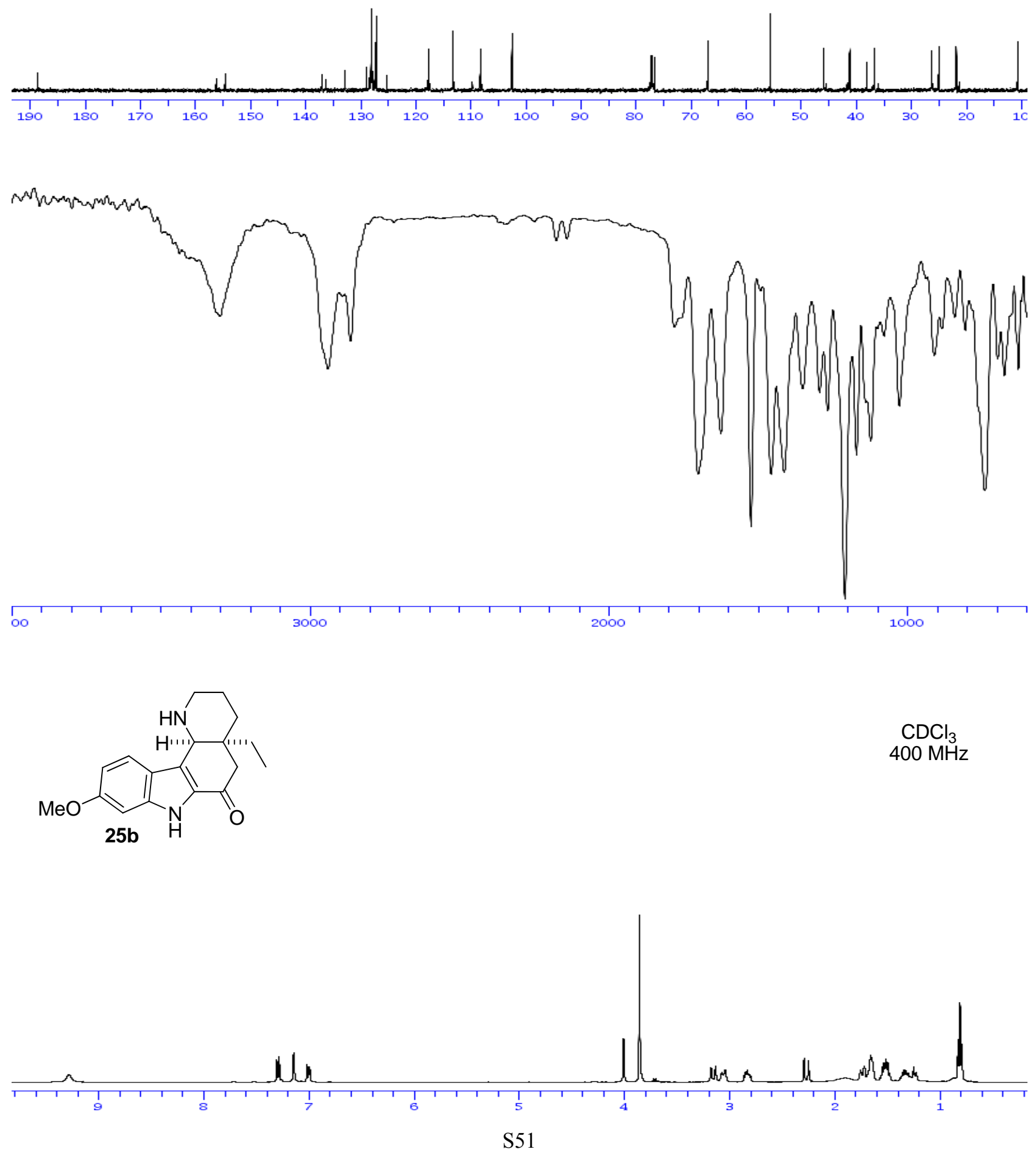
$\mathrm{CDCl}_{3}$

$100 \mathrm{MHz}$
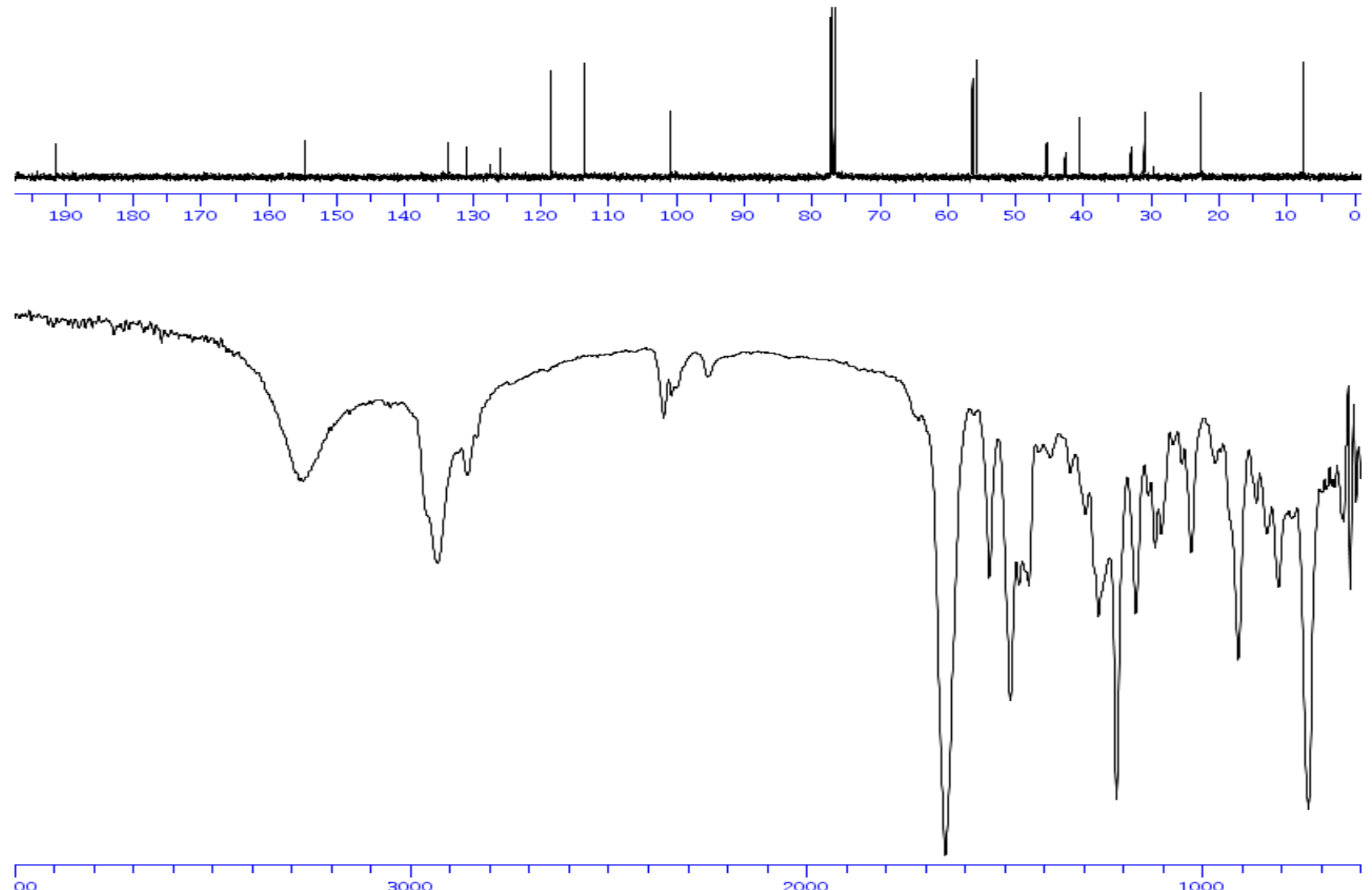

$\mathrm{MeO}$

$\mathrm{CDCl}_{3}$<smiles>COc1ccc2[nH]c(C(C)=O)cc2c1</smiles>
$400 \mathrm{MHz}$

26b<smiles>CC[C@@]1(C)CCCNC1C</smiles>

ull

the

....

dich

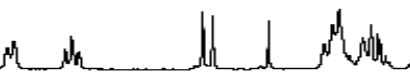
Wh 

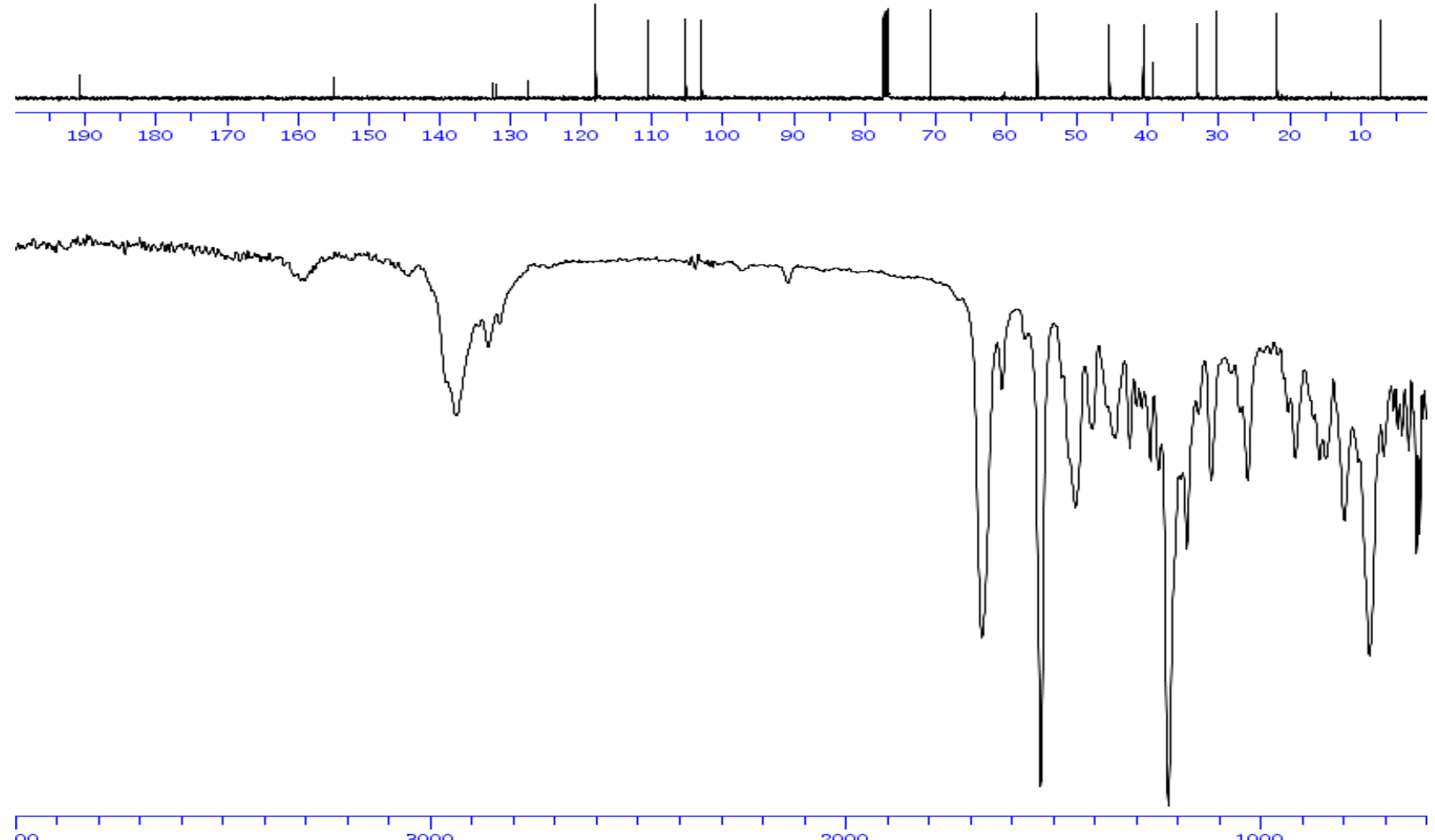<smiles>CCC12CCCN(C(=O)OCc3ccccc3)C1C2C(=O)c1cc2c(OC)cccc2[nH]1</smiles>

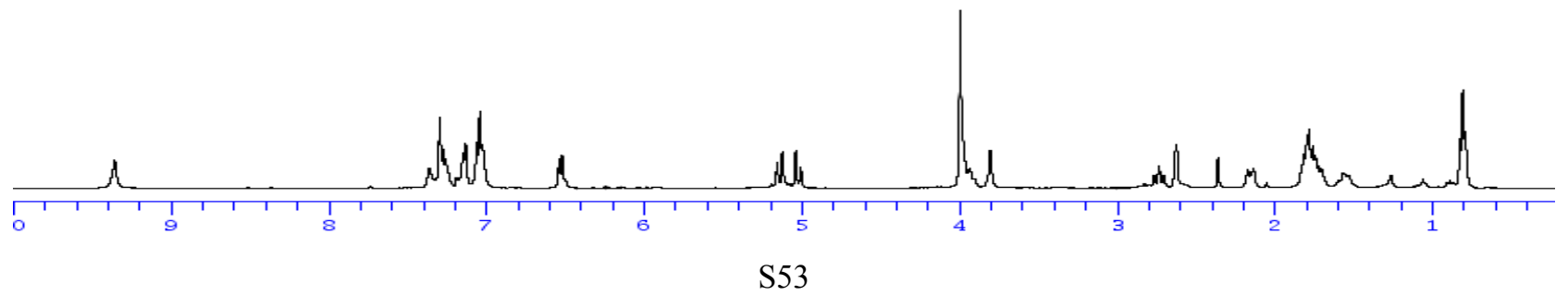



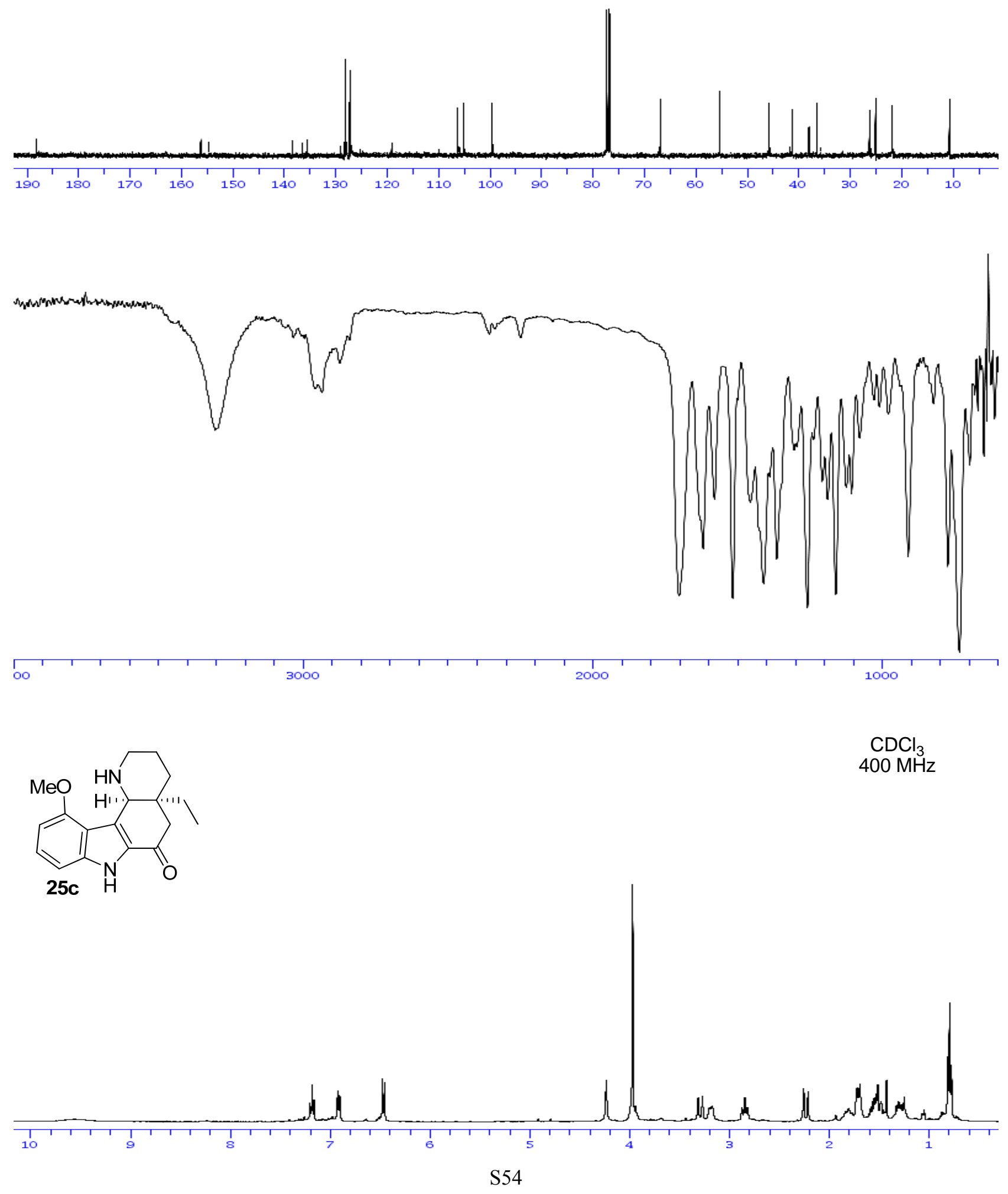
$\mathrm{CDCl}_{3}$
$100 \mathrm{MHz}^{-}$
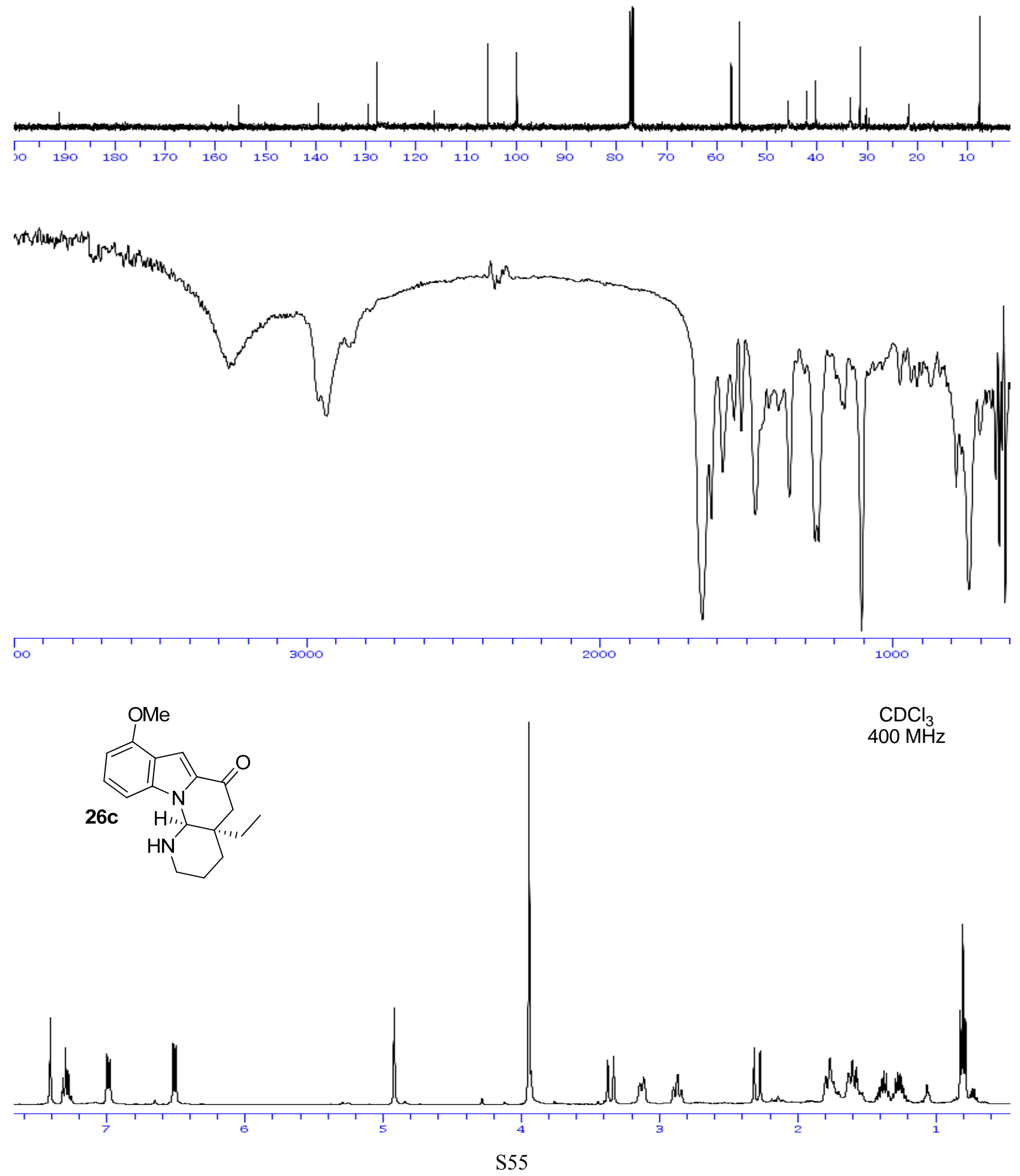

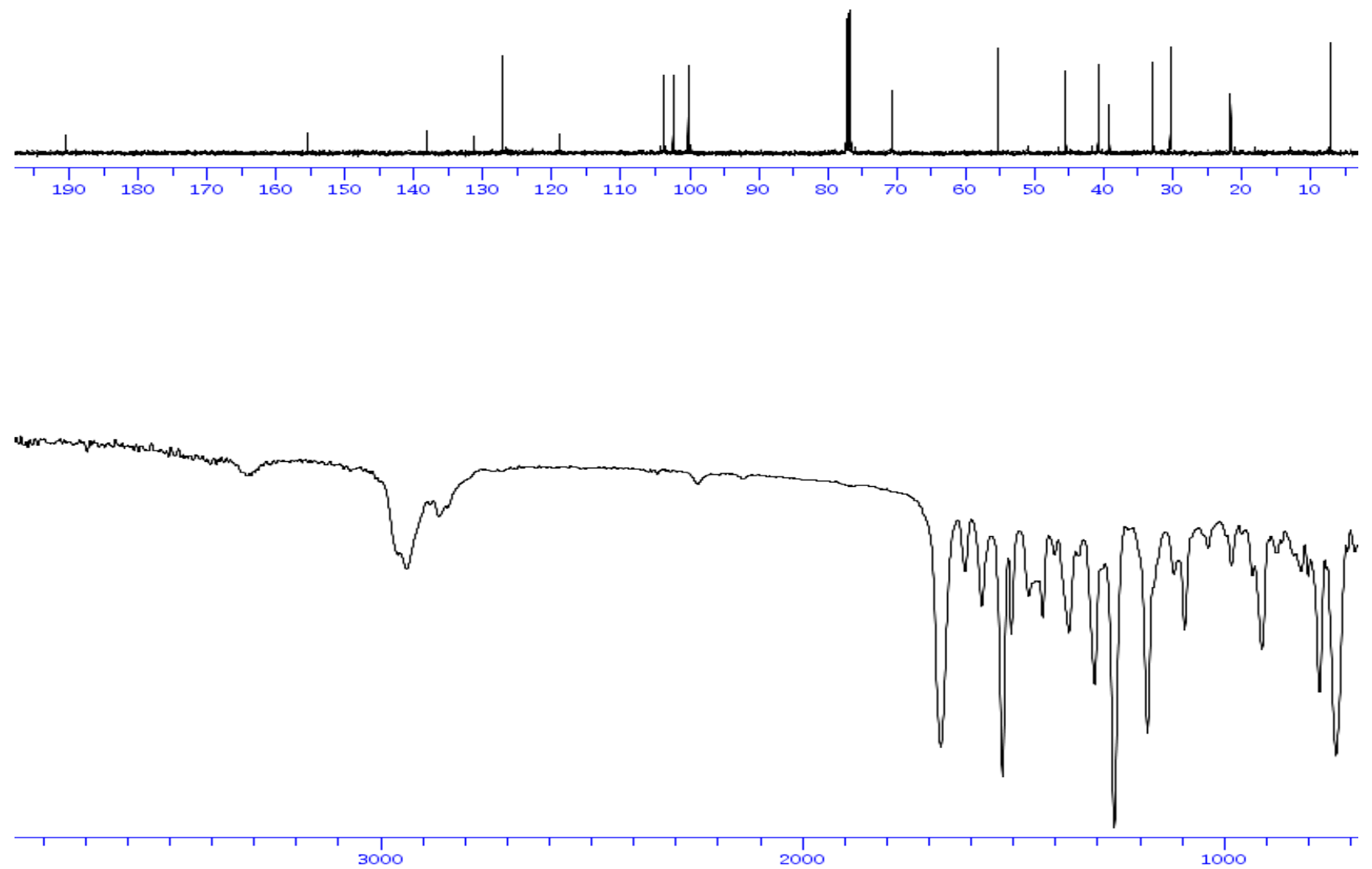<smiles></smiles>

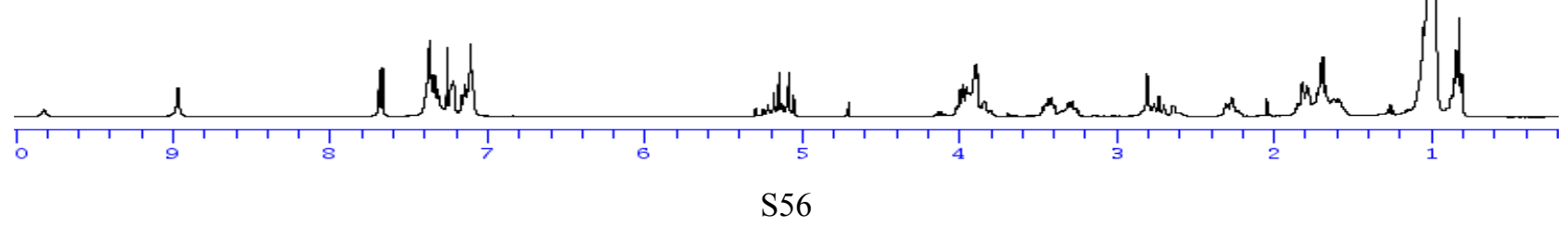



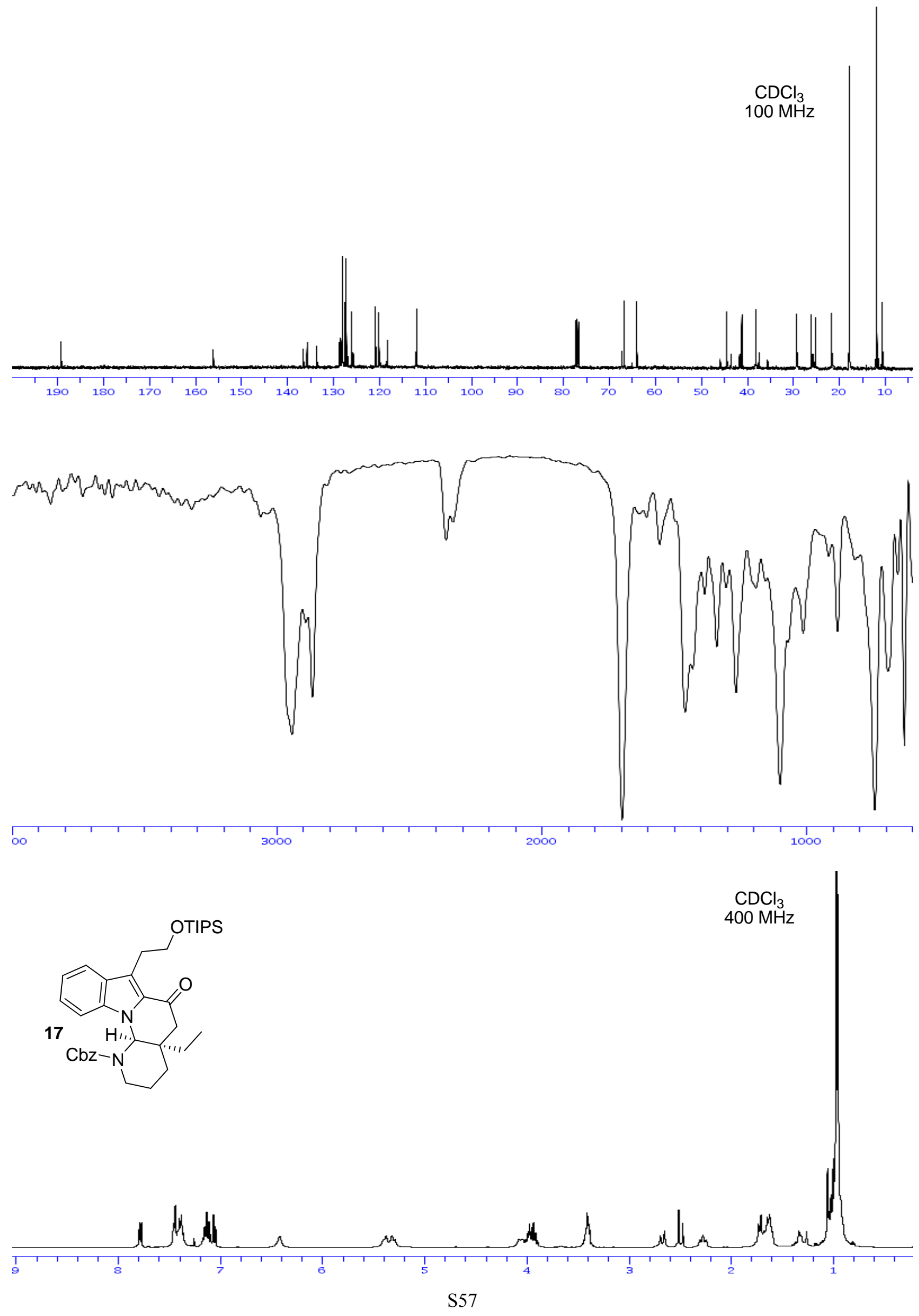

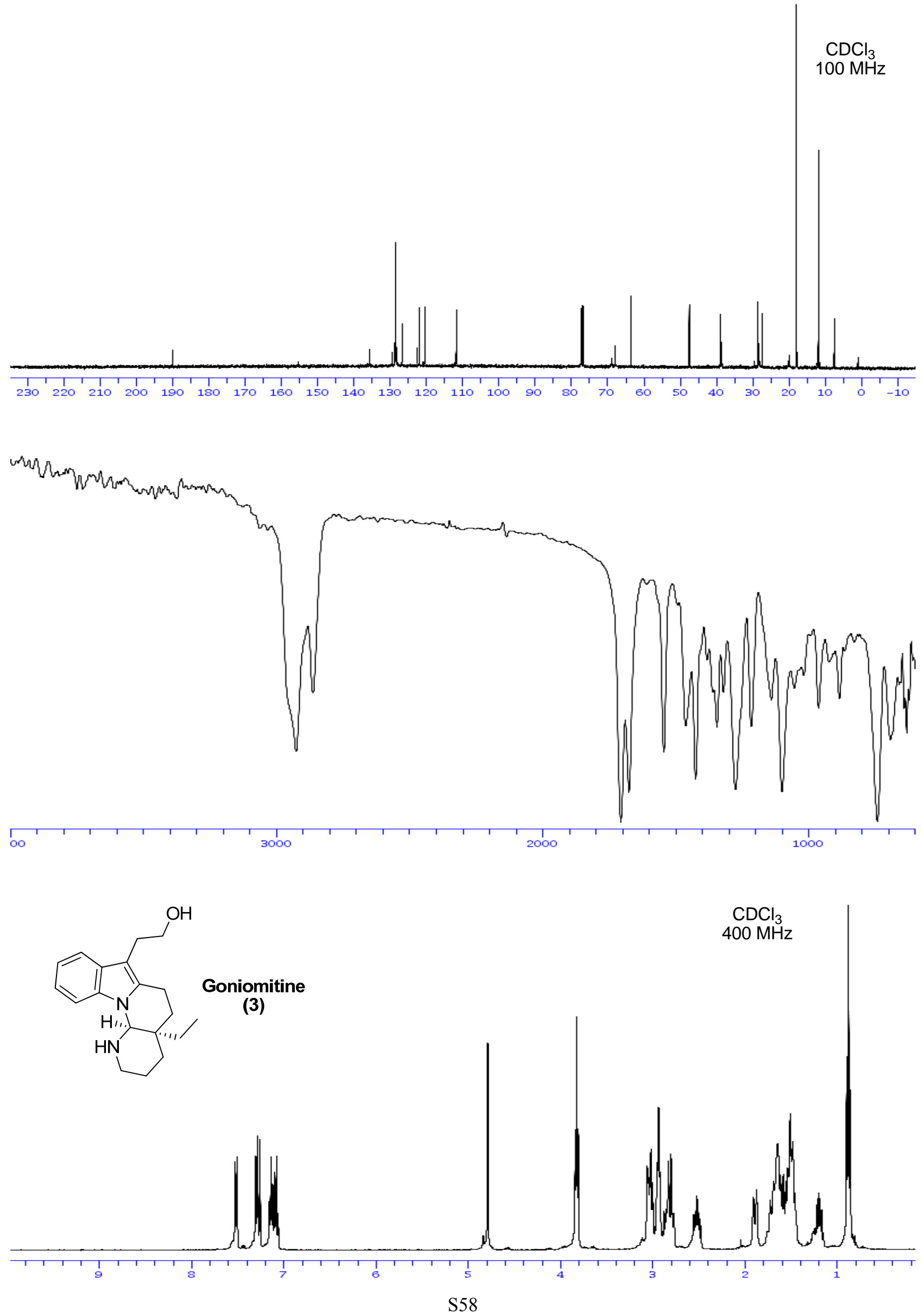


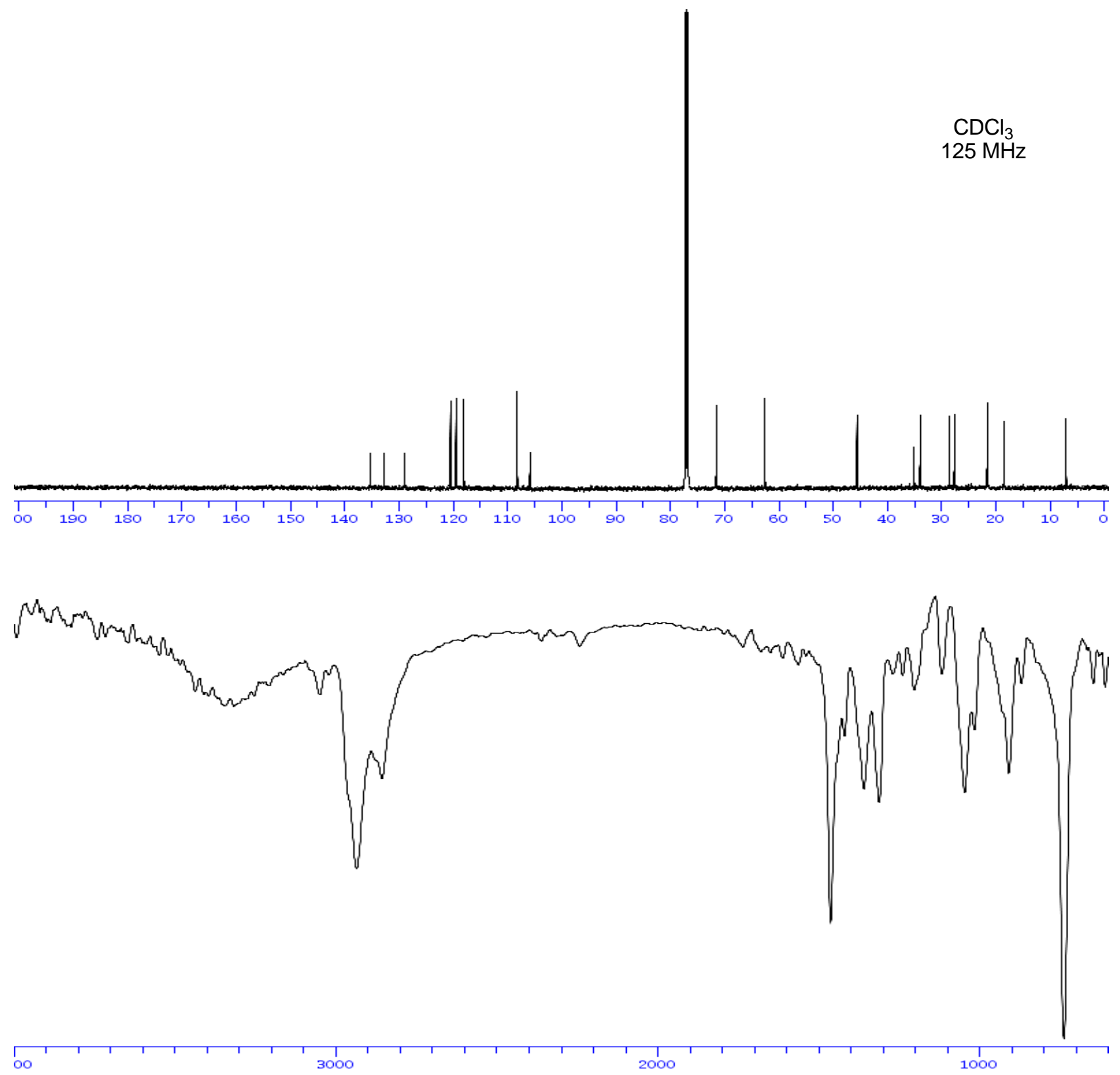


TimePrinted: 12/1/3910_10:13:5

title:fds384c-500 - 3

user: <fidesimo >

filename: $<$ fds $384 c-500>/ 1$

pulprog: $\langle$ zg_pi_pg.pv>

solvent: $\langle\mathrm{CDCl} 3>$

NS: 952

Frequency. $125.756532 \mathrm{MHz}$
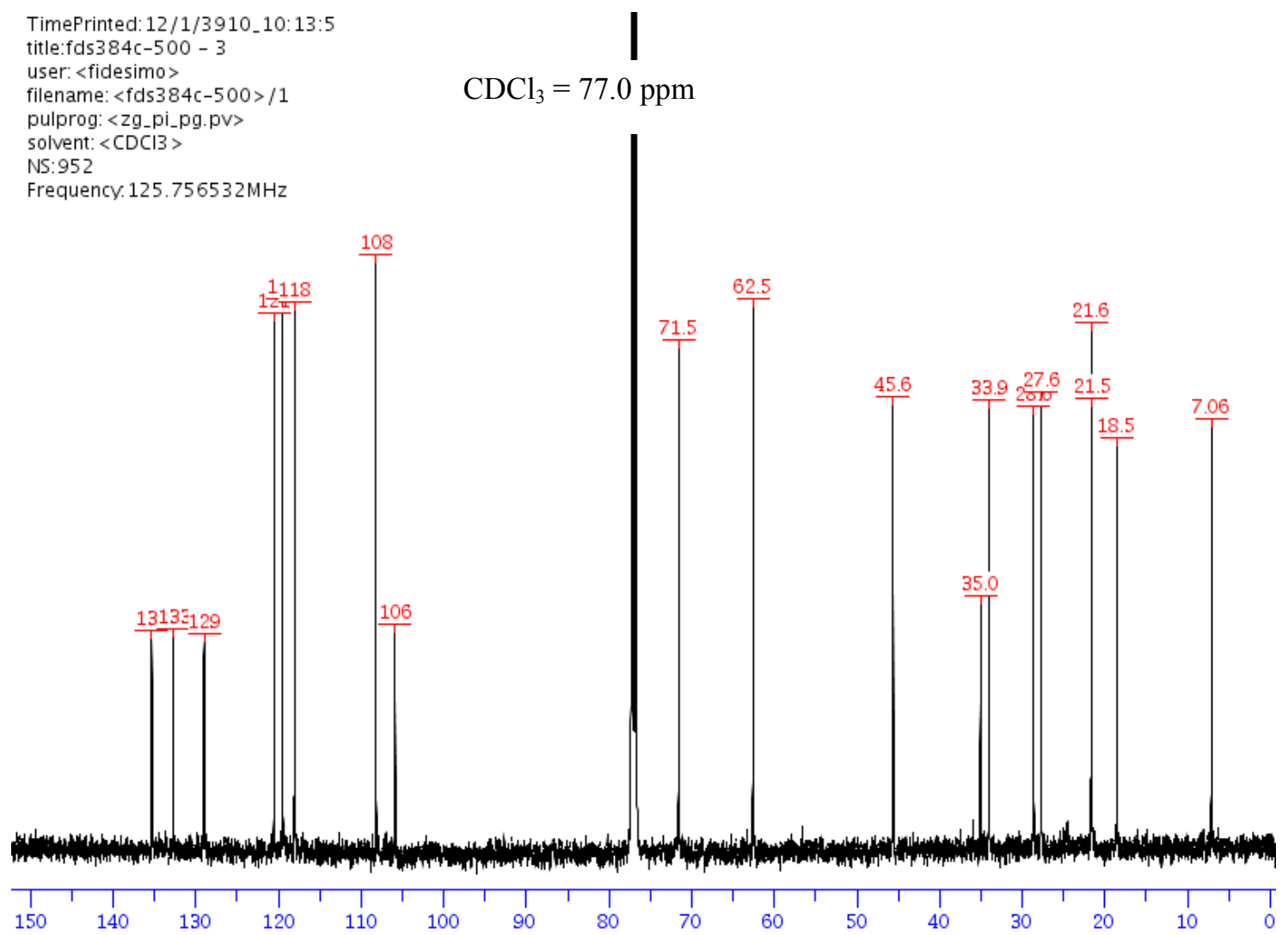

${ }^{13} \mathrm{C} \mathrm{NMR}\left(\mathrm{CDCl}_{3}, 125 \mathrm{MHz}\right) \delta 135.34,132.70,129.01,120.52,119.50,118.05,108.20,105.88,71.55,62.57$, $45.62,35.06,33.99,28.61,27.68,21.62,21.58,18.54,7.07$.

Pagenkopf (values kindly provided by Prof. Pagenkopf): ${ }^{[12 b]}{ }^{13} \mathrm{C} \mathrm{NMR}\left(\mathrm{CDCl}_{3}, 100 \mathrm{MHz}\right) \delta 135.35,132.70$, 129.04, 120.51, 119.51, 118.05, 108.18, 105.99, 71.51, 62.52, 35.07, 33.98, 28.61, 27.67, 21.65, 21.55, $18.52,7.05$.

Husson: ${ }^{[11]}{ }^{13} \mathrm{C} \mathrm{NMR}\left(\mathrm{CDCl}_{3}, 100 \mathrm{MHz}\right) \delta 135.4,132.6,129.3,120.8,119.9,118.1,108.7,106.8,71.1,62.6$, $45.4,35.3,33.8,28.7,27.8,21.8,20.8,18.5,7.3$. 


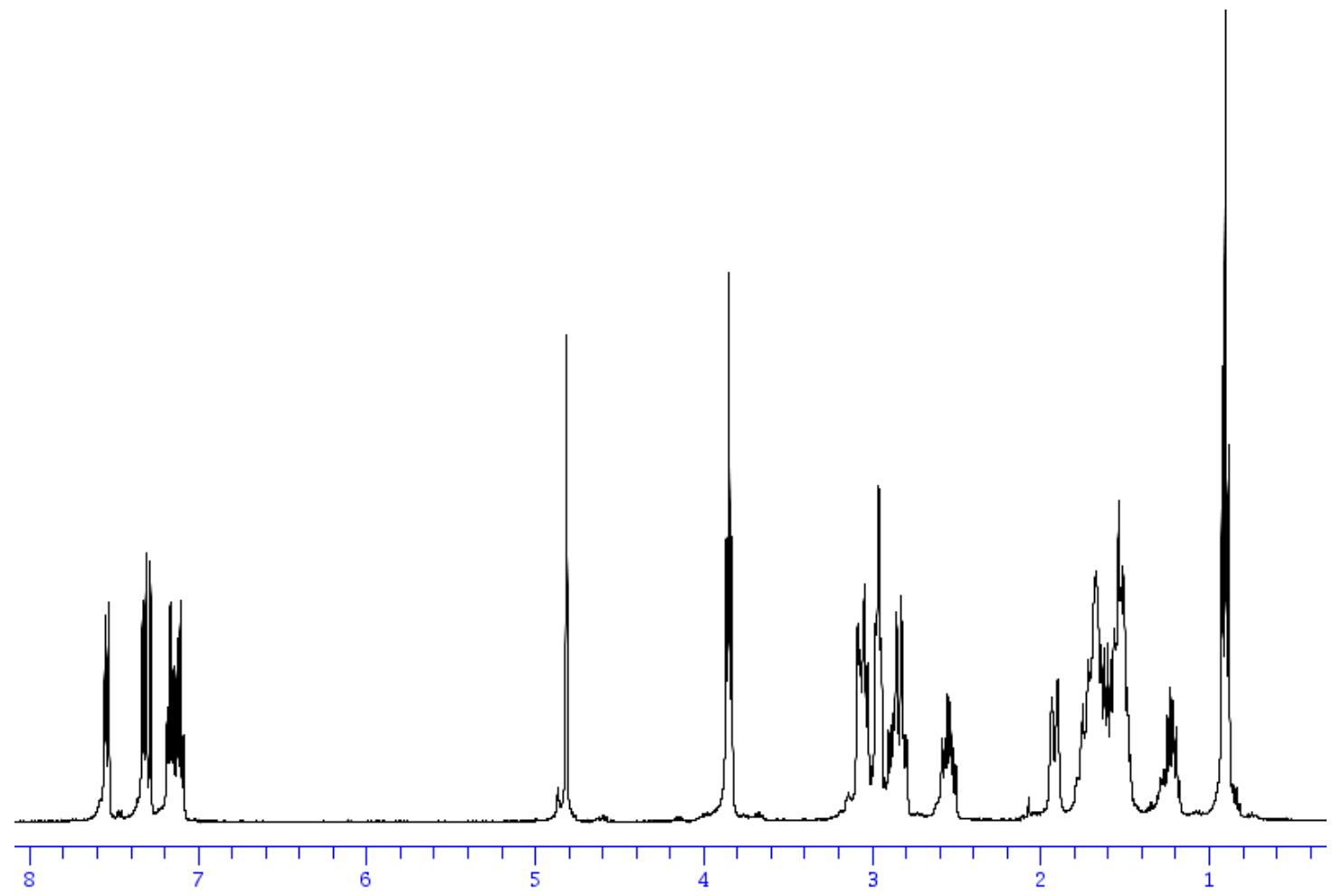

${ }^{1} \mathrm{H}$ NMR $\left(\mathrm{CDCl}_{3}, 400 \mathrm{MHz}\right) \delta 7.52$ (d, $J=7.7 \mathrm{~Hz}, 1 \mathrm{H}$; CH-Ar), 7.30 (d, $J=8.0 \mathrm{~Hz}, 1 \mathrm{H}$; CH-Ar), 7.14 (t, $J=7.2 \mathrm{~Hz}, 1 \mathrm{H}$; CH-Ar), 7.08 (t, $J=7.5 \mathrm{~Hz}, 1 \mathrm{H}$; CH-Ar), 4.79 (s, $1 \mathrm{H}$; NCH), 3.83 (t, $J=6.4 \mathrm{~Hz}, 2 \mathrm{H}$; $\left.\mathrm{OCH}_{2}\right), 3.12-2.75\left(\mathrm{~m}, 6 \mathrm{H} ; \mathrm{CH}_{2}\right), 2.52\left(\mathrm{td}, J=13.1 \mathrm{~Hz}, 6.7 \mathrm{~Hz}, 1 \mathrm{H} ; \mathrm{CH}_{2}\right), 1.89$ (d, $\left.J=13.8 \mathrm{~Hz}, 1 \mathrm{H} ; \mathrm{CH}_{2}\right)$, 1.80-1.41 (m, $7 \mathrm{H} ; \mathrm{CH}_{2} \mathrm{OH}$ and $\left.\mathrm{NH}\right), 1.21\left(\mathrm{~m}, 1 \mathrm{H} ; \mathrm{CH}_{2}\right), 0.88\left(\mathrm{t}, J=7.5 \mathrm{~Hz}, 3 \mathrm{H} ; \mathrm{CH}_{3}\right)$.

\section{Copy of Spectra from Husson:}

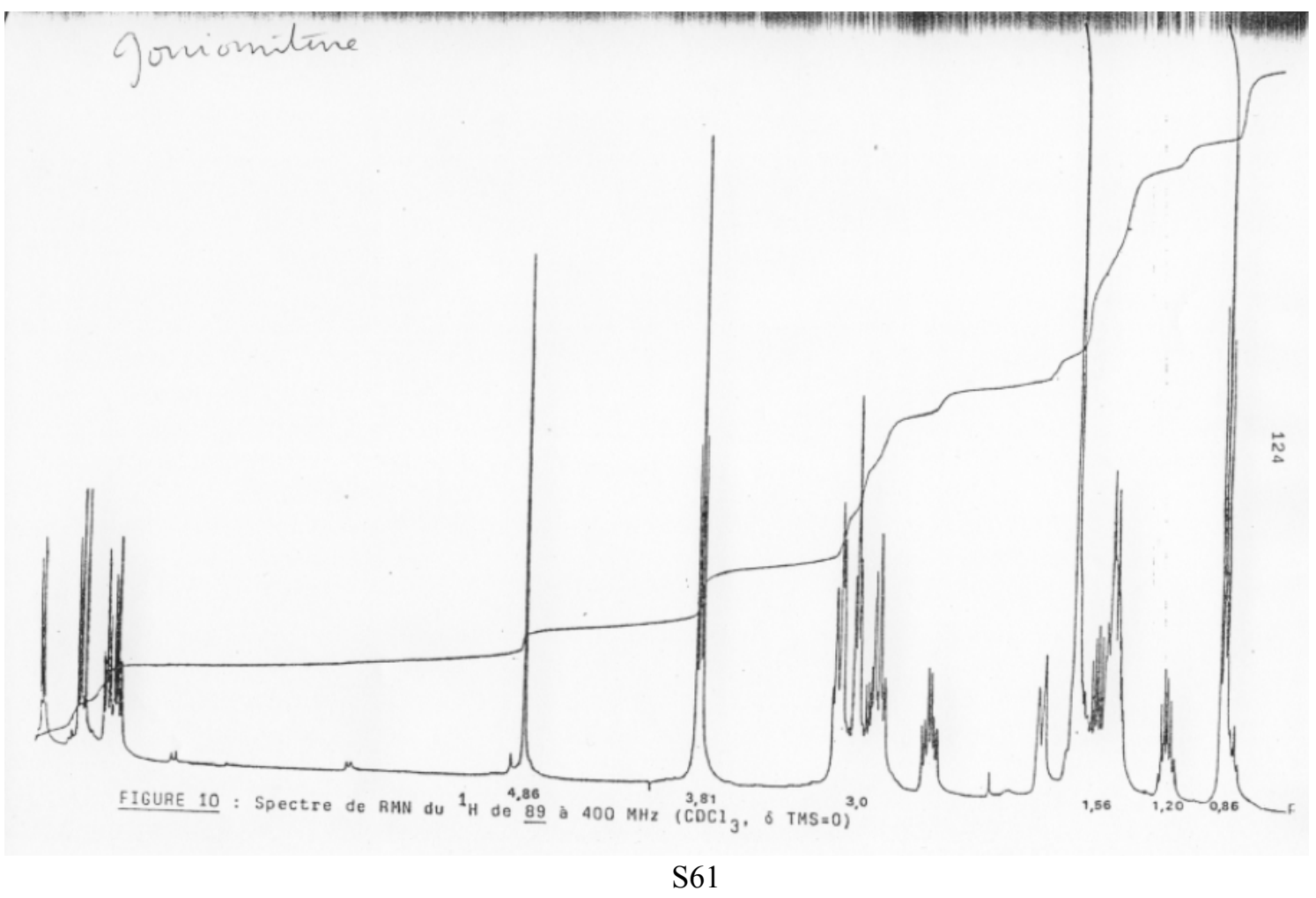

